\title{
Riemannian nilmanifolds, the wave trace, and the length spectrum
}

\author{
Ruth Gornet
}

\begin{abstract}
This paper examines the length spectrum on two-step nilmanifolds toward determining what, exactly, the wave trace says about isospectral manifolds. In particular, for each length occurring in the length spectrum of a two-step nilmanifold, we compute the leading order term in the associated wave invariant, under the assumption of the clean intersection hypothesis. En route, we calculate the Poincaré or First Return map for all two-step nilmanifolds. As an application, we explain certain examples of Heisenberg manifolds constructed by C.S. Gordon (C.S. Gordon, The Laplace spectra versus the length spectra of Riemannian manifolds, Contemporary mathematics: nonlinear problems in geometry (Mobile, Ala., 1985) vol. 51, AMS, 1986, pp. 63-80.) that are isospectral on functions, but have different multiplicities in the length spectrum. The multiplicity of a length is defined here as the number of free homotopy classes of loops that can be represented by a closed geodesic of that length.
\end{abstract}

\section{Introduction}

The spectrum of a closed Riemannian manifold $(M, g)$, denoted $\operatorname{spec}(M, g)$, is the collection of eigenvalues of the Laplace-Beltrami operator $\Delta$ acting on smooth functions. Two manifolds $(M, g)$ and $\left(M^{\prime}, g^{\prime}\right)$ are isospectral if $\operatorname{spec}(M, g)=\operatorname{spec}\left(M^{\prime}, g^{\prime}\right)$. The length spectrum of $(M, g)$, denoted by $\operatorname{spec}_{[L]}(M, g)$, is the collection of lengths of smoothly closed geodesics of $(M, g)$, counted with multiplicity. The multiplicity of a length is defined as the number of free homotopy classes of loops containing a closed geodesic of that length. The absolute length spectrum of a Riemannian manifold $(M, g)$, denoted $\operatorname{spec}_{L}(M, g)$, is the set of lengths of smoothly closed geodesics with no multiplicity assigned. (The absolute length spectrum is also referred to in the literature as the weak length spectrum.)

This material is based in part on work supported by NSF grant DMS-0204648 
A major open question in spectral geometry is the precise relation between the Laplace spectrum on functions and the (absolute) length spectrum. Using the heat equation, Colin de Verdière [5] has shown that generically (in the family of all Riemannian manifolds), the Laplace spectrum determines the absolute length spectrum. This result can also be obtained from the classical (wave) trace formula [11] (described below). In the case of compact, hyperbolic manifolds, this arises from the Selberg trace formula [40]. (See also [4, Chapter XI].)

In sharp contrast, Miatello and Rossetti [33] have constructed pairs of compact flat manifolds that are isospectral on one-forms but which do not have the same absolute length spectrum. (See also [6,7].) There is no known example of a pair of manifolds that are isospectral on functions but with unequal absolute length spectra.

Also in contrast, C.S. Gordon [14] has constructed pairs of isospectral Heisenberg manifolds that have unequal multiplicities in the length spectrum, and the author [21] has constructed other higher-step nilmanifolds with this property. These examples are of great interest, as it has been shown that all known methods for producing examples of isospectral nilmanifolds necessarily yield examples with the same absolute length spectrum [14,27]. A Riemannian two-step nilmanifold is a closed manifold of the form $(\Gamma \backslash G, g)$, where $G$ is a simply connected two-step nilpotent Lie group, $\Gamma$ is a uniform (i.e., $\Gamma \backslash G$ compact) discrete subgroup of $G$, and $g$ is a left invariant metric on $G$, which descends to a Riemannian metric on $\Gamma \backslash G$ that we also denote by $g$. A Heisenberg manifold is a two-step Riemannian nilmanifold whose covering Lie group $G$ is one of the $(2 n+1)$-dimensional Heisenberg Lie groups.

The purpose of this paper is to understand the behavior of the length spectrum on the isospectral Heisenberg manifolds of Gordon in particular, and on two-step nilmanifolds in general. Our approach is to compute certain wave invariants, which are defined below. En route to calculating the wave invariants on two-step nilmanifolds, we must calculate the DuistermaatGuillemin (DG) density. This in turn requires us to calculate the first return or Poincaré map. We apply these calculations to the isospectral Heisenberg manifolds of Gordon and show how a pair of manifolds can have the same Laplace spectrum, i.e., the same wave invariants, and yet have unequal multiplicities in the length spectrum. We conclude from these calculations that we cannot adjust the definition of the multiplicity of a length in the length spectrum in order to ensure that known examples of isospectral nilmanifolds must have the same length spectrum without making the multiplicity equal to the wave invariants. In other words, the only weighting of each length 
that assures equality is precisely the weighting given by the wave trace. See also Example 2.15 and Section 5.

For $(M, g)$ a Riemannian manifold, define

$$
e_{M}(t)=\operatorname{trace}(\exp (i t \sqrt{\Delta}))=\sum_{\lambda \in \operatorname{spec}(M, g)} e^{i t \sqrt{\lambda}}
$$

This is a tempered distribution that is determined by the Laplace spectrum. That is, if $M$ and $M^{\prime}$ are isospectral, then $e_{M}(t)=e_{M^{\prime}}(t)$. The classical trace formula, arising from the study of the wave equation, provides information about the singularities of $e_{M}(t)$. In particular [11],

(1) the singular support of $e_{M}(t)$ is contained in $\operatorname{spec}_{L}(M, g)$, and

(2) if $\tau$ is in $\operatorname{spec}_{L}(M, g)$ and $\tau$ satisfies a Clean Intersection Hypothesis, then $e_{M}(t)$ is a classical conormal distribution in a neighborhood of $\tau$, and the singularities of $e_{M}(t)$ at $\tau$ provide geometric information about $(M, g)$, the wave invariants.

See Section 2.1 below for more details about $e_{M}(t)$. Good references for distributions and singular support are [41, 42]. See also $[29,45]$.

The advantage of working with nilmanifolds is that they are "getatable" in the sense that the spectrum $[19,37]$ and the length spectrum $[12,26]$ are explicitly computable. Moreover, the generic results of Colin de Verdière and Duistermaat-Guillemin require that all closed geodesics be isolated and that lengths be multiplicity free. Nilmanifolds possess a great deal of symmetry so that closed geodesics always come in large dimensional families, thus failing these generic hypotheses. Consequently, generic results relating the Laplace and length spectra say nothing about the many known examples of isospectral nilmanifolds and other isospectral families of manifolds $[8,9,12$, 14-19, 21-24, 34-38].

This paper, while self-contained, is a continuation of [25], in which the author computes a necessary and sufficient condition for a two-step nilmanifold to satisfy the Clean Intersection Hypothesis. The computations of [25] are closely related to those here, and we use the necessary and sufficient condition to avoid two-step nilmanifolds failing the Clean Intersection Hypothesis in this paper. Of interest is the fact that the Heisenberg manifolds of Gordon that exhibit unequal multiplicities in the length spectrum are not related to the Clean Intersection Hypothesis. In particular, lengths of a Heisenberg manifold that are "unclean" are associated to free homotopy classes coming from central elements of the fundamental group. Whereas 
lengths of a Heisenberg manifold that exhibit unequal multiplicities in the length spectrum come from noncentral elements of the fundamental group.

This paper is organized as follows. In Section 2 we review necessary background information on the wave invariants and nilmanifolds. Large parts of Section 2 summarize material included in more detail in [25]. We include in Section 2 a warm up calculation of the wave invariants on the $n$-dimensional flat torus. We also include a summary of the computation of the wave invariants of interest on the Heisenberg manifolds of Gordon. Because of broad interest in Heisenberg manifolds, the author attempts to present this example so that a reader with a background in differential geometry may find it readable without getting bogged down with the many technical details of two-step nilmanifolds. In Section 3 we calculate the first return or Poincaré map and the DG-density for all two-step nilmanifolds. The Poincaré map is quite explicit, but we are not able to get a closed form for the DG-density in all cases. In Section 4 we examine other special cases, including the wave invariants on two-step nilmanifolds with a one-dimensional center/Heisenberg manifolds, and the DG-density for a specific five-dimensional example with a two-dimensional center. The five-dimensional example exhibits many of the subtleties possible in these calculations.

\section{Background}

\subsection{The wave invariants and the Duistermaat-Guillemin density}

The calculation of the wave invariants by DG uses the cotangent bundle [11]. As we are working on Riemannian manifolds, there is a natural (musical) identification between the cotangent bundle and the tangent bundle. The paper [30] has a nice expository explanation of the wave trace, and the set $F(\tau, c)$ here is equal to the set $W_{\tau}(c)$ there. We refer to the paper [3] for details about the calculation of the DG-measure. We refer to $[12,13]$ for details about the geodesic flow on nilmanifolds and the canonical symplectic form on the tangent bundle, when canonically (musically) identified with the cotangent bundle.

Denote by $\mathfrak{X}$ the unit tangent bundle of a Riemannian manifold $(M, g)$. For $v_{p} \in \mathfrak{X}$, denote by $\sigma_{v_{p}}(t)$ the unique geodesic in $(M, g)$ with initial velocity $v_{p}$. So $\sigma_{v_{p}}(0)=p \in M$, and $\sigma_{v_{p}}^{\prime}(0)=v_{p} \in \mathfrak{X}$. Denote geodesic flow on $(M, g)$ by $\Phi: \mathbb{R} \times \mathfrak{X} \rightarrow \mathfrak{X}$. So $\Phi\left(t, v_{p}\right)=\sigma_{v_{p}}^{\prime}(t)$. 
Denote by $c$ a free homotopy class of loops of $M$. For $\tau>0$, define

$$
F(\tau, c)=\left\{v_{p} \in \mathfrak{X}: \Phi\left(\tau, v_{p}\right)=v_{p}, \sigma_{\left.v_{p}\right|_{[0, \tau]}} \in c\right\}
$$

$F(\tau, c)$ is the set of all initial velocities producing smoothly closed, unit speed geodesics of period $\tau$ (i.e., of length $\tau$ ) that are, as closed loops, contained in the free homotopy class $c$. Note that $F(\tau, c)$ is empty if there does not exist a closed, unit speed geodesic in $c$ of length $\tau$.

For $\tau>0$, define $\Phi_{\tau}: \mathfrak{X} \rightarrow \mathfrak{X}$ by $\Phi_{\tau}\left(v_{p}\right)=\Phi\left(\tau, v_{p}\right)$. For $v_{p} \in F(\tau, c)$, define $\operatorname{FPS}\left(\tau, v_{p}\right)$ to be the fixed point set of $\Phi_{\tau *_{v_{p}}}$. Note that since $\Phi_{\tau}$ is the identity on $F(\tau, c)$, we must have $T_{v_{p}} F(\tau, c) \subset \operatorname{FPS}\left(\tau, v_{p}\right)$.

Definition 2.1. A period $\tau$ in $\operatorname{spec}_{L}(M, g)$ satisfies the Clean Intersection Hypothesis if for all $c$ such that $F(\tau, c)$ is nonempty, $F(\tau, c)=Z_{1} \cup \ldots \cup Z_{r}$, a finite union of submanifolds $Z_{k}$ of $\mathfrak{X}$, and for all $v_{p}$ in $F(\tau, c), T_{v_{p}} F(\tau, c)=$ $\operatorname{FPS}\left(\tau, v_{p}\right)$. The manifold $(M, g)$ satisfies the Clean Intersection Hypothesis if for all $\tau$ in $\operatorname{spec}_{L}(M, g), \tau$ satisfies the Clean Intersection Hypothesis.

For the remainder of this paper, we assume that the Clean Intersection Hypothesis is satisfied.

Recall that

$$
e_{M}(t)=\sum_{\lambda \in \operatorname{spec}(M, g)} e^{i t \sqrt{\lambda}} .
$$

Let $I$ be an interval in $\mathbb{R}$ such that $\operatorname{spec}_{L}(M, g) \cap I=\{\tau\}$. On the interval $I,[11$, p. 61]

$$
e_{M}(t)=\sum_{\substack{c \\ F(\tau, c) \neq \emptyset}} \sum_{j=1}^{r} \beta_{j}(t-\tau)
$$

where

$$
\beta_{j}(t)=\int_{-\infty}^{\infty} \alpha_{j}(s) e^{-i s t} d s
$$

and

$$
\alpha_{j}(s) \sim\left(\frac{s}{2 \pi i}\right)^{\left(d_{j}-1\right) / 2} i^{-\sigma_{j}} \sum_{k=0}^{\infty} \alpha_{j, k} s^{-k}
$$

as $s \rightarrow \infty, j=1, \ldots, r$. Here $d_{j}=\operatorname{dim} Z_{j}$, and $(1 / i)^{\left(d_{j}-1\right) / 2}=e^{-\pi i\left(d_{j}-1\right) / 4}$ if $d_{j}$ is even, $j=1, \ldots, r$. The value $\sigma_{j}$ can be identified as the Morse 
index for periodic geodesics in certain cases, see [10] and [11, pp. 69-70]. Duistermaat-Guillemin computed

$$
\alpha_{j, 0}=\frac{1}{2 \pi} \int_{Z_{j}} d \mu_{j},
$$

where $d \mu_{j}$ is the DG-density on $Z_{j}$.

A primary goal of this paper is to compute $d_{j}$ and $d \mu_{j}$ for (almost) all lengths in $\operatorname{spec}_{L}(M, g)$ in the special case that $M$ is a Riemannian two-step nilmanifold. The $Z_{j}$ were computed in [25, Theorem 3.8] (see Theorem 2.12 below) where we also computed an if and only if condition for a two-step nilmanifold to satisfy the Clean Intersection Hypothesis (see [25, Theorem 2.6] or Theorem 2.14 below). We use the computation of $d_{j}$ and $d \mu_{j}$ to calculate $\alpha_{j, 0}$ explicitly in the case of the isospectral Heisenberg manifolds of C.S. Gordon. (See Example 2.15 below.)

We now describe the DG-density. (Our exposition follows that of [3].) We must consider submanifolds of $T M$ rather than $\mathfrak{X}$ to capitalize on the symplectic structure. In order to do so, we amplify $F(\tau, c)$ to

$$
\hat{F}(\tau, c)=\left\{(\tilde{p}, t v):(\tilde{p}, v) \in F(\tau, c), t \in \mathbb{R}^{+}\right\} \subset T M .
$$

(See [11, p. 60, line-12 ff.].) For $v_{p} \in F(\tau, c)$, let $V=T_{v_{p}} T M$ and let $W=T_{v_{p}} \hat{F}(\tau, c)$. Note that $W \subset V$ depends on $\tau, c$ and $v_{p}$.

Recall that $T^{*} M$ has a canonical symplectic form $\Omega$. As $M$ is Riemannian, we can use $\Omega$ and the canonical (musical) isomorphism from $T^{*} M$ to $T M$ to obtain a canonical symplectic form on $T M$, which we also denote by $\Omega$.

Let $\mathcal{E}=\left\{e_{1}, \ldots, e_{K}\right\}$ be a basis of $W$. Let $\mathcal{V}=\left\{v_{1}, \ldots, v_{H}\right\}$ be a basis of a complement of $W$ in $V$. Denote by $W^{\perp}=\{v \in V: \Omega(v, w)=0, w \in W\}$, the symplectic orthogonal complement of $W$. Let $\mathcal{F}=\left\{f_{1}, \ldots, f_{K}\right\}$ be a basis of a complement of $W^{\perp}$ in $V$ such that $\Omega\left(e_{i}, f_{j}\right)=\delta_{i j}$.

Note that $\Phi_{\tau *_{v_{p}}}(V) \subset V$ since $v_{p} \in F(\tau, c)$. Consider the symplectomorphism $\Phi_{\tau *_{v_{p}}}: V \rightarrow V$. Define

$$
T=I-\Phi_{\tau *_{v_{p}}}
$$

By the Clean Intersection Hypothesis, $W=\operatorname{ker}(T)$. (See [11, p. 60, line-8].)

Lemma 2.2 [4, Lemma A.2]. The DG-density evaluated on $\mathcal{E}=$ $e_{1} \wedge \cdots \wedge e_{K}$ is

$$
\mu(\mathcal{E})=\frac{\nu(\mathcal{V} \wedge \mathcal{E})}{\nu(T \mathcal{V} \wedge \mathcal{F})},
$$

where $\mathcal{V}=v_{1} \wedge \ldots \wedge v_{H}, \mathcal{F}=f_{1} \wedge \ldots \wedge f_{K}, T \mathcal{V}=T v_{1} \wedge \ldots \wedge T v_{H}$ and $\nu$ is an arbitrary half density. 
Note that $T \mathcal{V}$ is a basis of $W^{\perp}$ (see [11, p. 60] or [3, p. 523]), and the expression in the Lemma is independent of the choice of half-density $\nu$ and the choice of $\mathcal{F}$ and $\mathcal{V}$. (Also note that for the $W$ considered in this paper, $W$ is not isotropic.)

Our approach will be to calculate $\alpha$ where $T \mathcal{V} \wedge \mathcal{F}=\alpha \mathcal{V} \wedge \mathcal{E}$. We then have

$$
\int_{Z_{j}} d \mu_{j}=\int_{Z_{j}} \frac{1}{\sqrt{|\alpha|}} d \sigma_{j}
$$

where $d \sigma_{j}$ is the Riemannian volume form on $Z_{j}$ coming from $\mathfrak{X}$. We refer to $\frac{1}{|\alpha|^{1 / 2}}$ as the $D G$-multiplier.

Finally, note that calculating $T \mathcal{V}$ requires calculating the derivative $\Phi_{\tau *_{v_{p}}}$ restricted to a complement of the fixed points of $\Phi_{\tau *_{v_{p}}}$. That is, we compute the Poincaré or first return map on a complement of $T_{v_{p}} \hat{F}(\tau, c)$ in $T M$.

\subsection{Riemannian two-step nilmanifolds and the first return map}

The manifolds we consider in this paper are constructed as follows. Let $G$ be a simply connected Lie group, and consider the action of $G$ on itself by left translations. Let $\Gamma$ be a cocompact (i.e., $\Gamma \backslash G$ compact), discrete subgroup of $G$; the existence of $\Gamma$ implies that $G$ is unimodular. A Riemannian metric $g$ is left invariant if the left translations $L_{p}$ of $G$ are isometries for all $p$ in $G$, so the action of $\Gamma$ on $G$ is by isometries. Note that a left invariant metric on $G$ is determined by specifying an inner product $\langle$,$\rangle on the Lie$ algebra $\mathfrak{g}$ of $G$ and vice versa. A Lie algebra together with an inner product $(\mathfrak{g},\langle\rangle$,$) is called a metric Lie algebra. The left invariant metric g$ descends to a Riemannian metric on $\Gamma \backslash G$, also denoted by $g$. With this metric, the mapping

$$
\pi_{1}:(G, g) \rightarrow(\Gamma \backslash G, g)
$$

is a Riemannian covering.

As is standard, we study closed geodesics on $(\Gamma \backslash G, g)$ by lifting to the universal cover $(G, g)$.

Definition 2.3. Let $\sigma$ be a unit speed geodesic of $(G, g)$. A nonidentity element $\gamma$ in $G$ translates $\sigma$ by an amount $\tau>0$ if $\gamma \sigma(t)=\sigma(t+\tau)$ for all $t \in \mathbb{R}$. The number $\tau$ is called a period of $\gamma$. 
As $G$ is simply connected, the free homotopy classes of $\Gamma \backslash G$ correspond to the conjugacy classes in the fundamental group $\Gamma$. We denote the conjugacy class in $\Gamma$ containing $\gamma \in \Gamma$ by $c_{\gamma}$. As $\pi_{1}$ is a Riemannian covering, we have the following:

Property 2.4. The manifold $(\Gamma \backslash G, g)$ contains a closed geodesic of length $\tau$ in the free homotopy class $c_{\gamma}$ if and only if the manifold $(G, g)$ contains a unit speed geodesic $\sigma(t)$ and there exists $\gamma^{\prime} \in c_{\gamma}$ such that $\gamma^{\prime}$ translates $\sigma$ with period $\tau$. Under the mapping $\pi_{1}$, the geodesic $\sigma$ then projects to a smoothly closed geodesic of length $\tau$ on $(\Gamma \backslash G, g)$ in the free homotopy class $c_{\gamma}$.

We likewise study the geodesic flow of $(\Gamma \backslash G, g)$ by first studying the geodesic flow of $(G, g)$. Let $\mathfrak{X}$ (resp. $\tilde{\mathfrak{X}}$ ) denote the unit tangent bundle of $\Gamma \backslash G(\operatorname{resp} . G)$.

Because the metric on $G$ is left invariant, the unit tangent bundle $\tilde{\mathfrak{X}}$ is equivalent to the sphere bundle, (viewing $\mathfrak{g}$ as $T_{e} G$ )

$$
\tilde{\mathfrak{X}} \cong G \times S^{n-1}=\{(\tilde{p}, v): \tilde{p} \in G, v \in \mathfrak{g},|v|=1\},
$$

under the mapping $v_{\tilde{p}} \mapsto\left(\tilde{p}, L_{\tilde{p}^{-1} *_{\tilde{p}}} v\right)$. We use $v_{\tilde{p}} \in \tilde{\mathfrak{X}}$ and $(\tilde{p}, v) \in G \times S^{n-1}$ interchangeably in what follows. ${ }^{*}$ Note that the left action of $G$ on $G$ by isometries determines an action of $G$ on $\tilde{\mathfrak{X}}$ by isometries:

$$
\tilde{q} \cdot v_{\tilde{p}}=\tilde{q} \cdot(\tilde{p}, v)=\left(L_{\tilde{q}}(\tilde{p}), v\right)=v_{\tilde{q} \tilde{p}}
$$

The subgroup $\Gamma$ then acts on $\tilde{\mathfrak{X}}$ by isometries as above, and

$$
\mathfrak{X} \cong \Gamma \backslash \tilde{\mathfrak{X}} \cong(\Gamma \backslash G) \times S^{n-1}
$$

We let $\pi$ denote the canonical mapping

$$
\pi: \tilde{\mathfrak{X}} \rightarrow \Gamma \backslash \tilde{\mathfrak{X}}
$$

so that $\pi_{1}$ defined in (2.6) is just $\pi$ restricted to the first factor.

Throughout this paper, elements with tildes such as $\tilde{p}, v_{\tilde{p}}$, denote elements in $G$ and $\tilde{\mathfrak{X}}$, respectively; elements without tildes such as $p$ and $v_{p}$ denote the image of $\tilde{p}$ and $v_{\tilde{p}}$ in $\Gamma \backslash G$ and $\mathfrak{X}$, respectively, under the canonical mapping $\pi: \tilde{\mathfrak{X}} \rightarrow \mathfrak{X}$. 
For $\tau>0$ and $\gamma \in \Gamma$, we define

$$
\tilde{F}(\tau, \gamma)=\left\{v_{\tilde{p}} \in \tilde{\mathfrak{X}}: \tilde{\Phi}_{\tau}\left(v_{\tilde{p}}\right)=\gamma \cdot v_{\tilde{p}}\right\}
$$

With these identifications, we have for the free homotopy class $c_{\gamma}$ and the length $\tau$ in the manifold $\Gamma \backslash G$, for any $\gamma^{\prime} \in c_{\gamma}$, (see [25, (1.13)])

$$
F\left(\tau, c_{\gamma}\right)=\pi\left(\tilde{F}\left(\tau, \gamma^{\prime}\right)\right)
$$

As $\tilde{\mathfrak{X}}$ is a Riemannian cover of $\mathfrak{X}$, we study $\Phi_{\tau *_{v_{p}}}$ by studying $\tilde{\Phi}_{\tau *_{\tilde{p}}}$ : $T_{v_{\tilde{p}}} \tilde{\mathfrak{X}} \rightarrow T_{\gamma \cdot v_{\tilde{p}}} \tilde{\mathfrak{X}}$ for $v_{\tilde{p}} \in \pi^{-1}\left(v_{p}\right)$. (See [25, Proposition $\left.1.15 \mathrm{ff}.\right]$.) Note that under the identification $\tilde{\mathfrak{X}} \cong G \times S^{n-1}$, we may write $T_{v_{\tilde{p}}} \tilde{\mathfrak{X}}$ as follows:

$$
T_{v_{\tilde{p}}} \tilde{\mathfrak{X}}=T_{\tilde{p}} G \times T_{v} S^{n-1}=\left\{\left(u, v^{\perp}\right): u \in T_{\tilde{p}} G, v^{\perp} \in \mathfrak{g}, v^{\perp} \perp v\right\},
$$

where by $S^{n-1}$ we mean the unit sphere in $\mathfrak{g}$. Likewise we have

$$
T_{v_{p}} \mathfrak{X}=T_{p}(\Gamma \backslash G) \times T_{v} S^{n-1}=\left\{\left(u, v^{\perp}\right): u \in T_{p}(\Gamma \backslash G), v^{\perp} \in \mathfrak{g}, v^{\perp} \perp v\right\} .
$$

We abuse notation slightly by identifying the components of $\pi_{*_{\tilde{p}}}\left(\left(u, v^{\perp}\right) v_{\tilde{p}}\right)=$ $\left(u, v^{\perp}\right)_{v_{p}}$ and $\left(u, v^{\perp}\right)_{v_{\tilde{p}}}$. We may do this since $\pi$ is a Riemannian covering, so $T_{p}(\Gamma \backslash G)$ and $T_{\tilde{p}} G$ are isometric vector spaces.

Example 2.5 Isospectral Flat Tori. The wave invariants are difficult to grasp intuitively, and as it is the focus of this paper, we present the following "warm-up" example. We calculate the wave invariant associated to an arbitrary element of the length spectrum of a flat torus.

We first summarize computations that are included in [25, Example 1.19]. Let $G=\mathbb{R}^{n}$. Here $\Gamma=L$, a lattice of full rank in $\mathbb{R}^{n}$. Then $L \backslash \mathbb{R}^{n}$ with the metric induced from the Euclidean metric on $\mathbb{R}^{n}$ is a closed Riemannian manifold. In fact, it is a one-step Riemannian nilmanifold. Note that both $L \backslash \mathbb{R}^{n}$ and $\mathbb{R}^{n}$ are Lie groups under addition and are endowed with a left (and right) invariant metric, as translations are isometries. As in the general case, we identify

$$
\tilde{\mathfrak{X}} \cong \mathbb{R}^{n} \times S^{n-1}=\left\{(\tilde{p}, v): \tilde{p} \in \mathbb{R}^{n}, v \in \mathbb{R}^{n},|v|=1\right\} .
$$

We continue to use $v_{\tilde{p}}$ and $(\tilde{p}, v)$ interchangeably and $v_{p}$ and $(p, v)$ interchangeably.

Geodesics in $\mathbb{R}^{n}$ are just straight lines, so geodesics in $L \backslash \mathbb{R}^{n}$ are projections of straight lines. For $v_{\tilde{p}} \in \tilde{\mathfrak{X}}, \sigma_{v_{\tilde{p}}}(t)=\tilde{p}+t v \in \mathbb{R}^{n}$ and $\tilde{\Phi}\left(t, v_{\tilde{p}}\right)=$ 
$v_{\tilde{p}+t v}=(\tilde{p}+t v, v)$. Since $\pi_{1}: \mathbb{R}^{n} \rightarrow L \backslash \mathbb{R}^{n}$ is a Riemannian submersion with totally geodesic fibers, for $v_{p} \in \mathfrak{X}, \Phi\left(t, v_{p}\right)=v_{p+t v}$.

Free homotopy classes of $L \backslash \mathbb{R}^{n}$ correspond to conjugacy classes in $L$. As $\mathbb{R}^{n}$ is abelian, the free homotopy classes of $L \backslash \mathbb{R}^{n}$ are in one-to-one correspondence with the elements of $L$. The unit speed geodesic $\sigma_{v_{\tilde{p}}}(t)$ on $\mathbb{R}^{n}$ projects to a closed geodesic of $L \backslash \mathbb{R}^{n}$ with period $\tau$ in the free homotopy class $c_{l}$ if and only if $\sigma_{v_{\tilde{p}}}(t+\tau)=l+\sigma_{v_{\tilde{p}}}(t)$ for all $t$. In this case $\tau=|l|$ and the length spectrum of $L \backslash \mathbb{R}^{n}$ is

$$
\operatorname{spec}_{[L]}\left(L \backslash \mathbb{R}^{n}\right)=\{|l|: l \in L\} .
$$

One easily computes that

$$
\tilde{F}(\tau, l)=\mathbb{R}^{n} \times\{l /|l|\} \text { and } F\left(\tau, c_{l}\right)=L \backslash \mathbb{R}^{n} \times\{l /|l|\} \quad \text { if }|l|=\tau .
$$

(See [25, Example 1.19] for more details.) Clearly, $F\left(\tau, c_{l}\right)$ is diffeomorphic to $L \backslash \mathbb{R}^{n}$, and $\operatorname{dim} \tilde{F}(\tau, l)=n=\operatorname{dim} F\left(\tau, c_{l}\right)$.

An arbitrary element of $T_{v_{p}} \mathfrak{X}$ that is normal to $T_{v_{p}} F\left(|l|, c_{l}\right)$ is given by $\left(0, v^{\perp}\right)_{v_{p}}$, where $v^{\perp}$ is any vector in $\mathbb{R}^{n}$ (viewed here as $\mathbb{R}^{n}=T_{e} \mathbb{R}^{n}$ ) that is orthogonal to $v$. (See (2.13).) Let $\alpha(s)=\left(p, \cos (s) v+\sin (s) v^{\perp}\right) \in \mathfrak{X}$. Then $\alpha(0)=(p, v)$, and $\dot{\alpha}(0)=\left(0, v^{\perp}\right) \in T_{v_{p}} \mathfrak{X}$. Also $\Phi_{\tau}(\alpha(0))=(p+\tau v, v)$, and

$$
\left.\frac{d}{d s}\right|_{\left.\right|_{0}} \Phi_{\tau}(\alpha(s))=\left(\tau v^{\perp}, v^{\perp}\right) \in T_{v_{p+\tau v}} \mathfrak{X} .
$$

But $p+\tau v=p+l=p$ in $L \backslash \mathbb{R}^{n}$, so

$$
\Phi_{\tau *_{v_{p}}}\left(\left(0, v^{\perp}\right)_{v_{p}}\right)=\left(\tau v^{\perp}, v^{\perp}\right)_{v_{p}} \in T_{v_{p}} \mathfrak{X}
$$

We now compute the wave invariants. Recall that the symplectic form on $T \mathbb{R}^{n}$, obtained from $T^{*} \mathbb{R}^{n}$ via the musical isomorphisms, is just

$$
\Omega\left((A, B),\left(A^{\prime}, B^{\prime}\right)\right)=\left\langle A, B^{\prime}\right\rangle-\left\langle B, A^{\prime}\right\rangle,
$$

for $A, B \in \mathbb{R}^{n}$.

Let $\left\{e_{1}, \ldots, e_{n}\right\}$ be an orthonormal basis of $\mathbb{R}^{n}$ such that $e_{1}=l /|l|$. After amplifying $\tilde{F}(\tau, l)$ as in $(2.4)$, we set

$$
\mathcal{E}=\left\{\left(e_{1}, 0\right), \ldots,\left(e_{n}, 0\right),\left(0, e_{1}\right)\right\} .
$$

Then using $\Omega$ and Lemma 2.2,

$$
\mathcal{F}=\left\{\left(0, e_{1}\right), \ldots,\left(0, e_{n}\right),\left(-e_{1}, 0\right)\right\}
$$


We set

$$
\mathcal{V}=\left\{\left(0, e_{2}\right), \ldots,\left(0, e_{n}\right)\right\}
$$

We must calculate $T \mathcal{V} \wedge \mathcal{F}$ as a multiple of $\mathcal{V} \wedge \mathcal{E}$, up to sign. Now $T \mathcal{V} \wedge \mathcal{F}$ equals

$$
\begin{gathered}
T\left(0, e_{2}\right) \wedge \cdots \wedge T\left(0, e_{n}\right) \wedge\left(0, e_{1}\right) \wedge \cdots \wedge\left(0, e_{n}\right) \wedge\left(-e_{1}, 0\right) \\
=\left(-\tau e_{2}, 0\right) \wedge \cdots \wedge\left(-\tau e_{n}, 0\right) \wedge\left(0, e_{1}\right) \wedge \cdots \wedge\left(0, e_{n}\right) \wedge\left(-e_{1}, 0\right) \\
= \pm \tau^{(n-1)} \mathcal{V} \wedge \mathcal{E} .
\end{gathered}
$$

Thus, the DG-multiplier is $\frac{1}{\tau^{(n-1) / 2}}$, and the wave invariant associated with $\tau=|l|$ and $l \in L$ is

$$
\operatorname{Wave}(|l|, l)=\frac{1}{(2 \pi i \tau)^{(n-1) / 2}} \operatorname{Vol}\left(L \backslash \mathbb{R}^{n}\right) .
$$

If we sum up over all free homotopy classes that contain a closed geodesic of length $\tau$, we obtain the wave invariant

$$
\operatorname{Wave}(\tau)=\frac{\operatorname{Vol}\left(L \backslash \mathbb{R}^{n}\right)}{(2 \pi i \tau)^{(n-1) / 2}} \sum_{l \in L,|l|=\tau} 1=\frac{\operatorname{mult}(\tau)}{(2 \pi i \tau)^{(n-1) / 2}} \operatorname{Vol}\left(L \backslash \mathbb{R}^{n}\right) .
$$

Note that we may add up over the free homotopy classes since the dimension of $\tilde{F}(\tau, l)$ is independent of the length $\tau$. Also, the Morse index for periodic geodesics is zero in this case.

See [48] for an alternate computation of the wave invariants of the flat torus.

Remark 2.6. In Example 2.15 below, we compute certain wave invariants on Heisenberg manifolds. In Section 4 we consider all of the wave invariants on Heisenberg manifolds, and we also explicitly compute the DG-multiplier in the case of a nontrivial five-dimensional example with a two-dimensional center.

\subsection{Two-step nilmanifolds and their geodesic flow}

For a Lie algebra $\mathfrak{g}$, denote by $\mathfrak{g}^{(1)}$ the derived algebra $[\mathfrak{g}, \mathfrak{g}]$ of $\mathfrak{g}$. A Lie algebra $\mathfrak{g}$ is two-step nilpotent if $\mathfrak{g}^{(1)} \subset \mathfrak{z}$, where $\mathfrak{z}$ denotes the center of $\mathfrak{g}$, and $\mathfrak{g}^{(1)} \not \equiv 0$. A Lie group $G$ is two-step nilpotent if its Lie algebra is. Let $\Gamma$ 
be a cocompact, discrete subgroup of a simply connected two-step nilpotent Lie group $G$ with left invariant metric $g$. The left invariant metric $g$ descends to a metric on $\Gamma \backslash G$ that we also denote by $g$. The locally homogeneous space $(\Gamma \backslash G, g)$ is called a Riemannian two-step nilmanifold.

Throughout the remainder of this paper, $G$ will denote a simply connected two-step nilpotent Lie group, $g$ will denote a left invariant metric on $G$, and $(\mathfrak{g},\langle\rangle$,$) will denote the associated metric Lie algebra. We denote$ the orthogonal complement of $\mathfrak{z}$ in $\mathfrak{g}$ by $\mathfrak{v}$ and write $\mathfrak{g}=\mathfrak{v} \oplus \mathfrak{z}$.

On all simply connected nilpotent Lie groups, the Lie group exponential $\exp : \mathfrak{g} \rightarrow G$ is a diffeomorphism [39], so $G$ is diffeomorphic to $\mathbb{R}^{n}$ where $n=\operatorname{dim} \mathfrak{g}$. For two-step nilpotent Lie groups, by the Campbell-BakerHausdorff formula [44], we may easily write the group operation of $G$ in terms of the Lie algebra $\mathfrak{g}$ by

$$
\exp (X) \exp (Y)=\exp \left(X+Y+\frac{1}{2}[X, Y]\right)
$$

where $X, Y \in \mathfrak{g}$. Thus,

$$
\begin{aligned}
\exp (X)^{-1} & =\exp (-X) \\
\exp (X) \exp (Y) \exp (X)^{-1} & =\exp (Y+[X, Y]), \\
\exp (X) \exp (Y) \exp (X)^{-1} \exp (Y)^{-1} & =\exp ([X, Y])
\end{aligned}
$$

Let $\log : G \rightarrow \mathfrak{g}$ denote the inverse of the diffeomorphism exp.

We use the following information about two-step nilpotent metric Lie algebras, which was first developed by Kaplan [31] for the study of Heisenberg groups.

Definition 2.7. Let $(\mathfrak{g},\langle\rangle$,$) be a two-step nilpotent metric Lie alge-$ bra, $\mathfrak{g}=\mathfrak{v} \oplus \mathfrak{z}$. Define a linear transformation $j: \mathfrak{z} \rightarrow s o(\mathfrak{v})$ by $j(Z) X=$ $(\operatorname{ad} X)^{*} Z$ for $Z \in \mathfrak{z}$ and $X \in \mathfrak{v}$. Equivalently, for each $Z \in \mathfrak{z}, j(Z): \mathfrak{v} \rightarrow \mathfrak{v}$ is the skew-symmetric linear transformation defined by

$$
\langle j(Z) X, Y\rangle=\langle Z,[X, Y]\rangle
$$

for all $X, Y$ in $\mathfrak{v}$. Here $\operatorname{ad} X(Y)=[X, Y]$ for all $X, Y \in \mathfrak{g}$, and $(\operatorname{ad} X)^{*}$ denotes the (metric) adjoint of $\operatorname{ad} X$.

By skew-symmetry, $j(Z)$ has $\operatorname{dim}_{\mathbb{R}}(\mathfrak{v})$ purely complex eigenvalues counting (algebraic) multiplicities and the nonzero eigenvalues occur in complex conjugate pairs; the eigenvalues of $j(Z)^{2}$ are then real and nonpositive. 
Each two-step nilpotent metric Lie algebra carries with it the $j$ operator. On the other hand, given inner product spaces $\mathfrak{v}$ and $\mathfrak{z}$ and a linear transformation $j: \mathfrak{z} \rightarrow s o(\mathfrak{v})$, one can define a two-step nilpotent metric Lie algebra $(\mathfrak{v} \oplus \mathfrak{z},\langle\rangle$,$) by requiring that \mathfrak{z}$ be central, that $\oplus$ be an orthogonal direct sum, and by defining the Lie bracket [, ] via (2.15). All two-step nilpotent metric Lie algebras are determined this way.

Definition 2.8. Let $(\mathfrak{g},\langle\rangle$,$) be a two-step nilpotent metric Lie algebra$ and let $Z \in \mathfrak{z}$.

(1) Let $\mu(Z)$ denote the number of distinct eigenvalues of $j(Z)^{2}$. For ease of notation, we write $\mu$ rather than $\mu(Z)$ when $Z$ is understood.

(2) Let $-\vartheta_{1}(Z)^{2},-\vartheta_{2}(Z)^{2}, \ldots,-\vartheta_{\mu}(Z)^{2}$ denote the $\mu$ distinct eigenvalues of $j(Z)^{2}$, with the assumption that $0 \leq \vartheta_{1}(Z)<\vartheta_{2}(Z)<\cdots<\vartheta_{\mu}(Z)$. The distinct eigenvalues of $j(Z)$ are then $\left\{ \pm \vartheta_{1}(Z) i, \ldots, \pm \vartheta_{\mu}(Z) i\right\}$.

(3) Define $\mathcal{M}(Z)=\{1, \ldots, \mu(Z)\}$ when $\operatorname{ker} j(Z)$ is trivial, and $\mathcal{M}(Z)=$ $\{2, \ldots, \mu(Z)\}$ when $\operatorname{ker} j(Z) \neq\{0\}$. We write $\mathcal{M}$ instead of $\mathcal{M}(Z)$ when $Z$ is understood.

(4) Let $W_{m}(Z)$ denote the invariant subspace of $j(Z)$ corresponding to $\vartheta_{m}(Z), \quad m=1, \ldots, \mu$. Then $j(Z)_{\mid W_{m}(Z)}^{2}=-\vartheta_{m}(Z)^{2} \operatorname{Id}_{\mid W_{m}(Z)}$; i.e., $W_{m}(Z)$ is the eigenspace of $j(Z)^{2}$ with eigenvalue $-\vartheta_{m}(Z)^{2}$. In particular, if $\vartheta_{1}(Z)=0$ then $W_{1}(Z)=\operatorname{ker} j(Z)$. By the skew-symmetry of $j(Z), \mathfrak{v}$ is the orthogonal direct sum of the invariant subspaces $W_{m}(Z)$ and we write

$$
\mathfrak{v}=\oplus_{m=1}^{\mu} W_{m}(Z)=\operatorname{ker} j(Z) \oplus_{m \in \mathcal{M}} W_{m}(Z) .
$$

(5) Note that if $m \in \mathcal{M}$, letting $J=j(Z)$ and $\vartheta_{m}=\vartheta_{m}(Z)$,

$$
\begin{gathered}
J_{\mid W_{m}(Z)}^{-1}=\frac{-1}{\vartheta_{m}^{2}} J_{\mid W_{m}(Z)} \\
e_{\mid W_{m}(Z)}^{s J}=\cos \left(s \vartheta_{m}\right) \operatorname{Id}+\frac{\sin \left(s \vartheta_{m}\right)}{\vartheta_{m}} J .
\end{gathered}
$$

Notation 2.9. Let $X_{0}+Z_{0}$ be a vector in $\mathfrak{g}$ with $X_{0} \in \mathfrak{v}$ and $Z_{0} \in \mathfrak{z}$.

(1) Define $X_{1}$ and $X_{2}$ by $X_{0}=X_{1}+X_{2}$ such that $X_{1} \in \operatorname{ker} j\left(Z_{0}\right)$ and $X_{2} \perp \operatorname{ker} j\left(Z_{0}\right)$.

(2) Let $\xi_{m}$ denote the component of $X_{2}$ in $W_{m}\left(Z_{0}\right)$, for each $m \in \mathcal{M}$. We write $X_{2}=\sum_{m \in \mathcal{M}} \xi_{m}$. If $W_{1}\left(Z_{0}\right)=\operatorname{ker} j\left(Z_{0}\right)$, then $\xi_{1}=0$. When necessary, we assume $j(Z)^{-1} \xi_{1}=0$ if $\vartheta_{1}(Z)=0$. 


\section{Definition 2.10.}

(1) For $V$ in $\mathfrak{v}$, define $P_{V}: \mathfrak{z} \rightarrow[V, \mathfrak{g}]$ as orthogonal projection onto $[V, \mathfrak{g}]$. Define $P_{V}^{\perp}: \mathfrak{z} \rightarrow[V, \mathfrak{g}]^{\perp}$ as projection onto $[V, \mathfrak{g}]^{\perp}$, the orthogonal complement of $[V, \mathfrak{g}]$ in $\mathfrak{z}$. For $V \in \mathfrak{v}$ and $Z \in \mathfrak{z}$, define $Z_{V}=P_{V}(Z)$ and $Z_{V}^{\perp}=P_{V}^{\perp}(Z)$.

(2) With notation as above, define $K: \mathfrak{g} \rightarrow \mathfrak{z}$ by

$$
K\left(X_{0}+Z_{0}\right)=Z_{0}+\frac{1}{2} \sum_{m \in \mathcal{M}}\left[j\left(Z_{0}\right)^{-1} \xi_{m}, \xi_{m}\right] .
$$

(3) Set $K_{V}=P_{V} \circ K$ and $K_{V}^{\perp}=P_{V}^{\perp} \circ K$.

The following theorem gives the length spectrum of an arbitrary twostep nilmanifold $(\Gamma \backslash G, g)$ by computing the periods of an arbitrary element of $\gamma \in \Gamma$.

Theorem 2.11 [26]. Let $G$ be a simply connected two-step nilpotent Lie group with left invariant metric $g$. Let $(\mathfrak{g},\langle\rangle$,$) be the associated metric Lie$ algebra. With notation as above, let $\gamma=\exp \left(V+Z_{V}+Z_{V}^{\perp}\right)$ be an element of $G$. The periods of $\gamma$ are precisely

$$
\left\{\sqrt{|V|^{2}+\frac{\left|Z_{V}^{\perp}\right|^{2}}{\left|K_{V}^{\perp}\left(X_{2}+Z_{0}\right)\right|^{2}}}: X_{2}+Z_{0} \text { satisfy (i)-(iv) below }\right\},
$$

where $\frac{\left|Z_{V}^{\perp}\right|}{\left|K_{V}^{\perp}\left(X_{2}+Z_{0}\right)\right|}=0$ if $Z_{V}^{\perp}=0$. Given $Z_{0} \in \mathfrak{z}, \mathcal{M}=\mathcal{M}\left(Z_{0}\right)$, and $X_{2}=$ $\sum_{m \in \mathcal{M}} \xi_{m} \in \mathfrak{v}$, where $\xi_{m} \in W_{m}\left(Z_{0}\right)$, the conditions referred to in $(\dagger)$ are the following:

(i) $\left|X_{2}+Z_{0}\right|=1$ or $X_{2}+Z_{0}=0$

(ii) $V \in \operatorname{ker} j\left(Z_{0}\right)$ and $X_{2} \perp \operatorname{ker} j\left(Z_{0}\right)$

(iii) $Z_{V}^{\perp} \in \operatorname{span}_{\mathbb{R}^{+}}\left\{K_{V}^{\perp}\left(X_{2}+Z_{0}\right)\right\}$

(iv) for all $m \in \mathcal{M}$ such that $\xi_{m} \neq 0, \frac{\left|Z_{V}^{\perp}\right| \vartheta_{m}\left(Z_{0}\right)}{2 \pi\left|K_{V}^{\perp}\left(X_{2}+Z_{0}\right)\right|} \in \mathbb{Z}$.

Let $\tau$ be a period of $\gamma=\exp \left(V+Z_{V}+Z_{V}^{\perp}\right)$. Define $\tau_{2}=\sqrt{\tau^{2}-|V|^{2}}$. The set $\tilde{F}(\tau, \gamma) \subset \tilde{\mathfrak{X}}$ is calculated as follows.

Theorem 2.12 [25]. Let $G$ be a simply connected two-step nilpotent Lie group with left invariant metric $g$. Let $(\mathfrak{g},\langle\rangle$,$) be the associated metric Lie$ 
algebra. Let $\gamma=\exp (V+Z) \in G$ and let $\tau>0$. With notation as above,

$$
\begin{aligned}
\tilde{F}(\tau, \gamma)= & \left\{v_{\tilde{p}}: v=\frac{1}{\tau}\left(V+\tau_{2}\left(X_{2}+Z_{0}\right)\right), \tilde{p}\right. \text { and } \\
& \left.X_{2}+Z_{0} \text { satisfy }(a)-(e) \text { below }\right\}
\end{aligned}
$$

Given $\tilde{p} \in G, Z_{0} \in \mathfrak{z}, \mathcal{M}=\mathcal{M}\left(Z_{0}\right)$ and $X_{2}=\sum_{m \in \mathcal{M}} \xi_{m} \in \mathfrak{v}$, where $\xi_{m} \in$ $W_{m}\left(Z_{0}\right)$, the conditions referred to in (- are the following:

(a) $\left|X_{2}+Z_{0}\right|=1$ or $X_{2}+Z_{0}=0$,

(b) $V \in \operatorname{ker} j\left(Z_{0}\right)$ and $X_{2} \perp \operatorname{ker} j\left(Z_{0}\right)$,

(c) $K_{V}^{\perp}\left(X_{2}+Z_{0}\right)=\frac{1}{\tau_{2}} Z_{V}^{\perp}$, where $\frac{1}{\tau_{2}} Z_{V}^{\perp}=0$ if $Z_{V}^{\perp}=0$,

(d) for all $m \in \mathcal{M}$ such that $\xi_{m} \neq 0, \frac{\tau_{2}}{2 \pi} \vartheta_{m}\left(Z_{0}\right) \in \mathbb{Z}$ and

(e) $[V, \log \tilde{p}]+Z_{V}=\left[V, j\left(Z_{0}\right)^{-1} X_{2}\right]+\tau_{2} K_{V}\left(X_{2}+Z_{0}\right)$, where $j\left(Z_{0}\right)^{-1} X_{2}=0$ if $X_{2}+Z_{0}=0$.

Note that $\tilde{F}(\tau, \gamma)=\emptyset$ if no such $\tilde{p}, X_{2}, Z_{0}$ exist.

Remark 2.13. Let $\gamma=\exp \left(V+Z_{V}+Z_{V}^{\perp}\right)$ as above. By the above theorems, if $Z_{V}^{\perp}=0$ then $\gamma$ has a unique period $\tau=|V|$. If $Z_{V}^{\perp} \neq 0$, by letting $X_{2}=0$ and $Z_{0}=Z_{V}^{\perp} /\left|Z_{V}^{\perp}\right|$, we conclude that $\tau=\left|V+Z_{V}^{\perp}\right|$ is always a period of $\gamma$, and is, in fact, the maximal period [12]. That is, if $\tau$ is a period of $\gamma$, then $\tau \leq\left|V+Z_{V}^{\perp}\right|$. If $\tau$ is a period of $\gamma$ and $\tau<\left|V+Z_{V}^{\perp}\right|$ and if $v \in \tilde{F}(\tau, \gamma)$ then

$$
v=\frac{1}{\tau}\left(V+\tau_{2}\left(X_{2}+Z_{0}\right)\right),
$$

with $X_{2} \neq 0$ and $\tau_{2}$ defined above.

The following theorem gives a necessary and sufficient condition for a length $\tau$ to satisfy the Clean Intersection Hypothesis.

Theorem 2.14 [25, Theorem 3.1]. Let $(\Gamma \backslash G, g)$ be a Riemannian twostep nilmanifold. With notation established above, $(\Gamma \backslash G, g)$ satisfies the Clean Intersection Hypothesis if and only if for all $\gamma=\exp (V+Z) \in \Gamma$ and for all $m \in \mathcal{M}\left(Z_{V}^{\perp}\right), \vartheta_{m}\left(Z_{V}^{\perp}\right) \notin 2 \pi \mathbb{Z}^{+}$. In particular, if $\tau \in \operatorname{spec}_{L}(\Gamma \backslash G, g)$ and there exists $\gamma \in \Gamma$ and $v_{p} \in F\left(\tau, c_{\gamma}\right)$ such that $T_{v_{p}} F\left(\tau, c_{\gamma}\right) \neq \operatorname{FPS}\left(\tau, v_{p}\right)$, then $\tau=\left|V+Z_{V}^{\perp}\right|$ and there exists $m \in \mathcal{M}\left(Z_{V}^{\perp}\right)$ such that $\vartheta_{m}\left(Z_{V}^{\perp}\right) \in 2 \pi \mathbb{Z}^{+}$. On the other hand, if $\gamma=\exp (V+Z) \in \Gamma$ and $\vartheta_{m}\left(Z_{V}^{\perp}\right) \in 2 \pi \mathbb{Z}^{+}$for some 
$m \in \mathcal{M}\left(Z_{V}^{\perp}\right)$, letting $\tau=\left|V+Z_{V}^{\perp}\right|$, then $F\left(\tau, c_{\gamma}\right) \neq \emptyset$ and $T_{v_{p}} F\left(\tau, c_{\gamma}\right) \neq$ $\operatorname{FPS}\left(\tau, v_{p}\right)$.

Example 2.15 Isospectral Heisenberg manifolds. In $[14,15,18]$ the geometry of isospectral Heisenberg manifolds was explored. These examples are a motivating factor for this paper.

Recall the $2 n+1$-dimensional Heisenberg group is defined as

$$
H_{n}=\left\{\left(\begin{array}{ccc}
1 & x & z \\
0 & I_{n} & y^{t} \\
0 & 0 & 1
\end{array}\right) x, y \in \mathbb{R}^{n}, z \in \mathbb{R}\right\}
$$

where $I_{n}$ is the $n \times n$ identity matrix. The group operation is determined by matrix multiplication. Letting $(x, y, z) \in \mathbb{R}^{2 n+1}$ be a coordinate system on $H_{n}$, we have $(x, y, z) \cdot\left(x^{\prime}, y^{\prime}, z^{\prime}\right)=\left(x+x^{\prime}, y+y^{\prime}, z+z^{\prime}+x y^{\prime}\right)$. A basis for the Lie algebra $\mathfrak{h}_{n}$ of $H_{n}$ is given by $\mathcal{B}=\left\{X_{1}, \ldots, X_{n}, Y_{1}, \ldots, Y_{n}, Z\right\}$ where in local coordinates, $X_{i}=\frac{\partial}{\partial x_{i}}, Y_{i}=\frac{\partial}{\partial y_{i}}+x_{i} \frac{\partial}{\partial z}$ and $Z=\frac{\partial}{\partial z}, i=1, \ldots, n$. The Lie bracket is given by $\left[X_{i}, Y_{j}\right]=\delta_{i j} Z$ and all other basis brackets determined by skew-symmetry or equal to zero, $i, j=1, \ldots, n$. We specify a left invariant metric $g$ for $H_{n}$ by specifying an orthonormal basis of $\mathfrak{h}_{n}$. Without loss of generality $[14,15,18]$ we may assume that this orthonormal basis is of the form

$$
\left\{\frac{1}{\sqrt{a_{1}}} X_{1}, \ldots, \frac{1}{\sqrt{a_{n}}} X_{n}, \frac{1}{\sqrt{a_{1}}} Y_{1}, \ldots, \frac{1}{\sqrt{a_{n}}} Y_{n}, Z\right\},
$$

where $0<a_{1} \leq a_{2} \leq \cdots \leq a_{n}$.

Define $\Pi: H_{n} \rightarrow \mathbb{R}^{2 n}$ by $\Pi(x, y, z)=(x, y)$. We specify the metric $\bar{g}$ on $\mathbb{R}^{2 n}$ by restricting $g$ to an orthogonal complement of the center $\operatorname{span}\{Z\}$ of $\mathfrak{h}_{n}$. That is, we view $\mathbb{R}^{2 n}$ as $\operatorname{span}\left\{X_{1}, \ldots, X_{n}, Y_{1}, \ldots, Y_{n}\right\}$ with inner product determined by specifying $\left\{\frac{1}{\sqrt{a_{1}}} X_{1}, \ldots, \frac{1}{\sqrt{a_{n}}} X_{n}, \frac{1}{\sqrt{a_{1}}} Y_{1}, \ldots, \frac{1}{\sqrt{a_{n}}} Y_{n}\right\}$ as an orthonormal basis. With this choice of metric, $\Pi$ is a Riemannian submersion with totally geodesic fibers. Note that we abuse notation by identifying elements of $\mathbb{R}^{2 n}$ and elements of the Lie algebra of $\mathbb{R}^{2 n}$.

Let $\Gamma$ be a cocompact, discrete subgroup of $H_{n}$. Let $L=\Pi(\Gamma)$, which is a cocompact, discrete subgroup of $\mathbb{R}^{2 n}$; i.e., $L$ is a lattice in $\mathbb{R}^{2 n}$. Let $c>0$ be such that $\Gamma \cap Z\left(H_{n}\right)=\{(0,0, c k): k \in \mathbb{Z}\}$. We write $\Gamma=\Gamma(L, c)$. While $L$ and $c$ do not completely determine $\Gamma$ as a set, $L$ and $c$ do determine the isometry class of $\Gamma$. That is, if $\Gamma=\Gamma(L, c)$ and $\Gamma^{\prime}=\Gamma^{\prime}(L, c)$, then $\left(\Gamma \backslash H_{n}, g\right)$ and $\left(\Gamma^{\prime} \backslash H_{n}, g\right)$ are isometric $[14,15,18]$. We call $\left(L \backslash \mathbb{R}^{2 n}, \bar{g}\right)$ the associated torus of $\left(\Gamma \backslash H_{n}, g\right)$. 
We may now present the construction used by Gordon and Gordon and Wilson to construct (nontrivial) pairs of isospectral Heisenberg manifolds.

Theorem 2.16 [15, Propositions 2.14 and 2.16], [43, Proposition III.5 and Remark III.8]. Every Riemannian Heisenberg manifold is isometric to one of the form $\left(\Gamma \backslash H_{n}, g\right)$ where $\Gamma=\Gamma(L, c)$ and $g$ descends from a left invariant metric on $H_{n}$ that is determined by specifying the orthonormal basis of the type $(h)$, where $0<a_{1} \leq a_{2} \leq \ldots \leq a_{n}$. Two manifolds $\left(\Gamma \backslash H_{n}, g\right)$ and $\left(\Gamma^{\prime} \backslash H_{n}, g^{\prime}\right)$ of this form are isometric if and only if $g=g^{\prime}$ and $\Gamma^{\prime}=\Phi(\Gamma)$ for some automorphism $\Phi$ of $H_{n}$ such that $\Phi^{*} g=g$. The manifolds $\left(\Gamma \backslash H_{n}, g\right)$ to $\left(\Gamma^{\prime} \backslash H_{n}, g^{\prime}\right)$ of this form are $\Delta$-isospectral if and only if $c=c^{\prime}, a_{i}=a_{i}^{\prime}, i=1, \ldots, n$ (i.e., $\left.g=g^{\prime}\right)$, and the associated tori $\left(L \backslash \mathbb{R}^{2 n}, \bar{g}\right)$ and $\left(L^{\prime} \backslash \mathbb{R}^{2 n}, \bar{g}\right)$ are $\Delta$-isospectral.

We now show by direct computation that isospectral Heisenberg manifolds must have the same wave invariants. This must be true, as the wave invariants are a spectral invariant. However, it is of interest to see exactly what geometric information is contained in the wave invariants.

Let $\left(\Gamma \backslash H_{n}, g\right)$ and $\left(\Gamma^{\prime} \backslash H_{n}, g\right)$ be isospectral Heisenberg manifolds. By Theorem 2.16, $\Gamma=\Gamma(L, c)$ and $\Gamma^{\prime}=\Gamma\left(L^{\prime}, c\right)$ where $\left(L \backslash \mathbb{R}^{2 n}, \bar{g}\right)$ and $\left(L^{\prime} \backslash \mathbb{R}^{2 n}\right.$, $\bar{g})$ are $\Delta$-isospectral. Note that since volume is a spectral invariant, we must have $\operatorname{Vol}\left(\Gamma \backslash H_{n}, g\right)=\operatorname{Vol}\left(\Gamma^{\prime} \backslash H_{n}, g\right)$.

We may decompose the length spectrum of $\left(\Gamma \backslash H_{n}, g\right)$ as follows. Recall from above that lengths of closed geodesics in $\left(\Gamma \backslash H_{n}, g\right)$ are determined by the periods of $\gamma$-periodic geodesics on $H_{n}$. Let $\gamma \in \Gamma(L, c)$, so $\gamma=(x, y, c k)$ where $\Pi(\gamma) \in L$ and $k \in \mathbb{Z}$.

Because $\Gamma$ and $\Gamma^{\prime}$ have the same center, and because $\left(H_{n}, g\right)$ covers both manifolds, the lengths and multiplicities coming from periods of central elements must correspond. It follows from (4.2) and (4.3) below, which are computed in Section 4.1, that the associated wave invariants must likewise correspond, i.e., the DG-multipliers are equal and the resulting volume calculations must be equal as well, as they depend only on $\operatorname{Vol}\left(\Gamma \backslash H_{n}, g\right)=$ $\operatorname{Vol}\left(\Gamma^{\prime} \backslash H_{n}, g\right)$ and the $a_{i}, i=1, \ldots, n$. See Remark 4.1 at the end of Section 4.1 for more details.

We thus restrict our attention here to the case where a length or period $\tau$ arises from a noncentral element $\gamma$ of $\Gamma$, i.e., $\gamma \sigma(s)=\sigma(s+\tau)$ where $\Pi(\gamma)=$ $l_{0} \neq 0$ and $\sigma$ is a unit speed geodesic on $H_{n}$. 
We write $\gamma=\left(l_{0}, *\right)$, and we know $\tau=\left|l_{0}\right|$. (See Remark 2.13.) We show below in (I-DG) that the DG-multiplier in this case is

$$
\frac{1}{\tau^{(2 n-1) / 2}\left|J l_{0}\right|},
$$

where $J$ is defined in Definition 2.7 above. Let $N=J l_{0} /\left|J l_{0}\right|$. Then $N$ is a unit normal to $C\left(\gamma, H_{n}\right)$, the centralizer of $\gamma$ in $H_{n}$, since $x \in C\left(\gamma, H_{n}\right)$ if and only if $\log x \perp N$. Here, $\log : H_{n} \rightarrow \mathfrak{h}_{n}$ is the inverse of exp $: \mathfrak{h}_{n} \rightarrow$ $H_{n}$ and is given by $\log (x, y, z)=\sum_{i}\left(x_{i} X_{i}+y_{i} Y_{i}\right)+\left(z-\frac{1}{2} x \cdot y\right) Z$. By [9, Appendix A],

$$
\operatorname{Vol}(\Gamma \backslash \tilde{F}(\tau, \gamma))=\operatorname{Vol}\left(C(\gamma, \Gamma) \backslash C\left(\gamma, H_{n}\right)\right)=p_{l_{0}}^{-1} \operatorname{Vol}\left(\Gamma \backslash H_{n}\right)
$$

where

$$
p_{l_{0}}=\min _{\gamma \in \Gamma}{ }^{+} \operatorname{proj}(\log \gamma, N)=\frac{\min _{l \in L}^{+}\left|\left\langle l, J l_{0}\right\rangle\right|}{\left|J l_{0}\right|},
$$

where by $\min ^{+}$we mean the least positive element and by $\operatorname{proj}(\log \gamma, N)$ the vector projection of $\log \gamma$ onto $N$. Note that the dimension of $\Gamma \backslash \tilde{F}(\tau, \gamma)$ is $2 n$.

The wave invariant associated with an element $\gamma=\left(l_{0}, *\right)$ and period $\tau=\left|l_{0}\right|$ is then

$$
\operatorname{Wave}(\tau, \gamma)=\frac{1}{(2 \pi \tau i)^{(2 n-1) / 2} p_{l_{0}}\left|J l_{0}\right|} \operatorname{Vol}\left(\Gamma \backslash H_{n}\right)
$$

One can compute that the number of free homotopy classes of loops $c_{\gamma}$ with $\Pi(\gamma)=l_{0}$ is $p_{l_{0}}\left|J l_{0}\right| / c,[9,(\mathrm{~A} .10)]$. We must sum up over these $p_{l_{0}}\left|J l_{0}\right| / c$ free homotopy classes of loops, and furthermore sum up over all $l^{\prime} \in L$ such that $\left|l^{\prime}\right|=\left|l_{0}\right|$, obtaining a wave invariant for $\tau=\left|l_{0}\right|$ of

$$
\operatorname{Wave}(\tau)=\frac{\overline{\operatorname{mult}}(\tau)}{(2 \pi \tau i)^{(2 n-1) / 2} c} \operatorname{Vol}\left(\Gamma \backslash H_{n}\right),
$$

where $\overline{\operatorname{mult}}(\tau)$ is the multiplicity of the length $\tau$ in $\operatorname{spec}_{[L]}\left(L \backslash \mathbb{R}^{2 n}\right)$. Note that by Theorem 2.16, isospectral Heisenberg manifolds must have isospectral associated tori. It is well known (see for example [2]) that $L \backslash \mathbb{R}^{2 n}$ and $L^{\prime} \backslash \mathbb{R}^{2 n}$ are isospectral if and only if they have the same length spectrum; i.e., there exists a length preserving bijection from $L$ onto $L^{\prime}$.

We conclude that isospectral Heisenberg manifolds must have the same wave invariants. 
There is no reason to assume that the length $\tau=\left|l_{0}\right|$ is not additionally the period of a central lattice element. If this is the case, then the leading term associated to $\tau$ will not come from the noncentral fixed point set. This is because the dimension of the fixed point set for a central element will always be larger than for a noncentral element, so the leading term of the singularity at $\tau$ will come from geometric information from the central fixed point set.

We are now able to explain the examples of isospectral Heisenberg manifolds with different multiplicities in the length spectrum. Recall that the multiplicity of a length $\tau$ is the number of distinct free homotopy classes of loops that can be represented by a closed geodesic of length $\tau$. As free homotopy classes of loops correspond to conjugacy classes in $\Gamma$, in the case of a $\tau$ not appearing in a central free homotopy class, this amounts to counting the number of free homotopy classes $c_{\gamma}$ with $|\Pi(\gamma)|=\tau$, which equals

$$
\operatorname{mult}(\tau)=\sum_{\substack{l \in L \\|l|=\tau}} \sum_{\substack{c \gamma \in \Gamma \\ \Pi(\gamma)=l}} 1=\frac{1}{c} \sum_{\substack{l \in L \\|l|=\tau}} p_{l}|J(l)| .
$$

There is no reason to believe the right-hand side of this equation is a spectral invariant, and indeed, in [14], Gordon gives explicit examples to show that it is not.

However, when we weight each free homotopy class with the value of the wave invariant (2.16), we then end up with a spectral invariant, the wave invariant!

Throughout the remainder of the paper, a dot denotes a derivative with respect to $s$, and a prime denotes a derivative with respect to $t$ or $r$.

For $\gamma=\exp \left(V+Z_{V}+Z_{V}^{\perp}\right)$ with period $\tau$, let $v_{\tilde{p}} \in \tilde{F}(\tau, \gamma)$. Our goal is to calculate $\Phi_{\tau *_{v_{p}}}$, i.e., the first return or Poincaré map. To do so, we need the following.

Proposition 2.17. Let $G$ be a two-step nilpotent Lie group with left invariant metric $g$. Let $\gamma=\exp (V+Z) \in G$ have period $\tau$, and let $v_{\tilde{p}} \in$ $\tilde{F}(\tau, \gamma)$. Let $\alpha(s)=\left(\tilde{p} \exp \left(a_{1}(s)\right), a_{2}(s)\right) \in \tilde{\mathfrak{X}}$ such that $a_{1}(0)=0$ and $a_{2}(0)=v$. Let $a_{2}(s)=X_{0}^{s}+Z_{0}^{s}$ with $Z_{0}^{s} \in \mathfrak{z}$ and $X_{0}^{s} \in \mathfrak{v}$. Let $\exp \left(X^{s}(t)+\right.$ $\left.Z^{s}(t)\right)$ be the geodesic through $e$ with initial velocity $a_{2}(s)$. Then the 
Poincaré map satisfies

$$
\begin{aligned}
\tilde{\Phi}_{\tau *_{\tilde{p}}}(\dot{\alpha}(0))= & \tilde{\Phi}_{\tau *_{\tilde{p}}}\left(\dot{a}_{1}(0), \dot{a}_{2}(0)\right)=\frac{d}{\left.d s\right|_{0}} \tilde{\Phi}_{\tau}(\alpha(s)) \\
= & \left(\dot{a}_{1}(0)+\dot{X}^{0}(\tau)+\dot{Z}^{0}(\tau)\right. \\
& \left.+\left[\dot{a}_{1}(0)+\frac{1}{2} \dot{X}^{0}(\tau), V\right] ; \dot{X}^{\prime} 0(\tau)+\dot{Z}_{0}^{0}\right) . \\
\left(I-\tilde{\Phi}_{\tau *_{\tilde{p}}}\right)(\dot{\alpha}(0))= & \left(I-\tilde{\Phi}_{\tau *_{\tilde{p}}}\right)\left(\dot{a}_{1}(0), \dot{a}_{2}(0)\right) \\
= & \left(-\dot{X}^{0}(\tau)-\dot{Z}^{0}(\tau)\right. \\
& \left.+\left[V, \dot{a}_{1}(0)+\frac{1}{2} \dot{X}^{0}(\tau)\right] ;-\dot{X}^{\prime} 0(\tau)+\dot{X}_{0}^{0}\right) .
\end{aligned}
$$

Also, $\tilde{\Phi}_{\tau *_{\tilde{p}}}(\dot{\alpha}(0)) \neq \gamma \cdot \dot{\alpha}(0)$ if and only if $\dot{X}^{0}(\tau) \neq 0$ or $\dot{Z}^{0}(\tau) \neq\left[V, \dot{a}_{1}(0)\right]$ or $\dot{X^{\prime} 0}(\tau) \neq \dot{X}_{0}^{0}$.

Proof of Proposition. This follows directly from the proof of [25, Proposition 3.11]. Note that the conditions (1)-(3) of [25, Proposition 3.11] are equivalent to $\tilde{\Phi}_{\tau}\left(v_{\tilde{p}}\right)=\gamma \cdot v_{\tilde{p}}$, which must be satisfied since $v_{\tilde{p}} \in \tilde{F}(\tau, \gamma)$.

In order to use Proposition 2.17 to calculate $\tilde{\Phi}_{\tau *_{\tilde{p}}}(X)$ for $X \notin T_{v_{\tilde{p}}} \tilde{F}(\tau, \gamma)$, we must calculate $\dot{X}^{0}(\tau), \dot{Z}^{0}(\tau)$ and $\dot{X}^{\prime} 0(\tau)$, where $\exp \left(X^{s}(\tau)+Z^{s}(\tau)\right)$ is the geodesic through $e$ with initial velocity $a_{2}(s)=X_{0}^{s}+Z_{0}^{s}$, where $Z_{0}^{s} \in \mathfrak{z}$ and $X_{0}^{s} \in \mathfrak{v}$. Because we will be taking limits and derivatives of vector curves $X_{0}^{s}+Z_{0}^{s}$ in $\mathfrak{g}$, and because the number of distinct eigenvalues of $j\left(Z_{0}^{s}\right)$ can vary with $s$, we will need the following definitions and properties.

Definition 2.18. Let $(\mathfrak{g},\langle\rangle$,$) be a two-step nilpotent metric Lie algebra.$ Define $\mathcal{U}=\{Z \in \mathfrak{z}$ : there exists an open neighborhood $\mathcal{O}$ of $Z$ such that $\mu$ is constant on $\mathcal{O}\}$. We call $\mathcal{U}$ the simple subdomain of $\mathfrak{z}$.

Proposition 2.19 [26, Proposition 1.19]. Let $(\mathfrak{g},\langle\rangle$,$) be a two-step$ nilpotent metric Lie algebra. Then the following hold.

(1) The simple subdomain $\mathcal{U}$ is open and dense in $\mathfrak{z}$.

(2) The function $\mu(Z)$ is constant on $\mathcal{U}$.

(3) The function $\vartheta_{m}: \mathcal{U} \rightarrow \mathbb{R}$ is smooth on $\mathcal{U}-\{0\}$ for $m=1, \ldots, \mu(Z)$.

(4) If $Z$ is a limit point of $\mathcal{U}$, then $\mu(Z) \leq \mu(\mathcal{U})$. 
First assume that $Z_{0}=\lim _{s \rightarrow 0} Z_{s} \in \mathcal{U}$. We may then assume that the number of distinct eigenvalues of $j\left(Z_{s}\right)$ is constant for $s$ in a neighborhood of 0 . Let $X_{1}^{s}$ denote the component of $X_{0}^{s}$ in $\operatorname{ker} j\left(Z_{s}\right)$. Let $X_{2}^{s}$ denote the component of $X_{0}^{s}$ orthogonal to $\operatorname{ker} j\left(Z_{s}\right)$, and let $\xi_{m}^{s}$ denote the component of $X_{2}^{s}$ in $W_{m}\left(Z_{s}\right)$, for $m \in \mathcal{M}=\mathcal{M}\left(Z_{0}\right)=\mathcal{M}\left(Z_{s}\right)$. By [1], if $Z_{s}$ and $X_{0}^{s}$ are analytic in $s$, we know that the invariant subspace $W_{m}\left(Z_{s}\right)$, the eigenvalue curves $\vartheta_{m}\left(Z_{s}\right)$ and the eigenvector curves $X_{1}^{s}, X_{2}^{s}$ and $\xi_{m}^{s}$ are analytic in $s$, $m \in \mathcal{M}$. Thus all of the limits and derivatives exist below.

If $Z_{0} \notin \mathcal{U}$, then we must be more precise. As $\mathcal{U}$ is dense in $\mathfrak{z}, Z_{0}$ is a limit point of $\mathcal{U}$. By Proposition 2.19 and continuity of the set of (unordered) eigenvalues [32, §II.5], two of the eigenvalue curves must approach each other as $Z$ approaches $Z_{0} \in \mathfrak{z}-\mathcal{U}$ and the counting function $\mu$ has a discontinuity at $Z_{0}$. We proceed as though exactly two eigenvalue curves $\vartheta_{m^{\prime}}$ and $\vartheta_{m^{\prime \prime}}$ intersect at $Z_{0}$; the statements generalize in the obvious manner in the case that more than two eigenvalue curves intersect at $Z_{0}$. As $\mathcal{U}$ is dense and open in $\mathfrak{z}$, there exist $Z_{s} \rightarrow Z_{0}, Z_{s} \in \mathcal{U}$, such that $\frac{d}{d s \mid}{ }_{\mid 0} Z_{s}$ takes any desired value, and such that as $s \rightarrow 0$,

$$
\lim _{s \rightarrow 0} \vartheta_{m^{\prime}}\left(Z_{s}\right)=\vartheta_{m}\left(Z_{0}\right) \text { and } \lim _{s \rightarrow 0} \vartheta_{m^{\prime \prime}}\left(Z_{s}\right)=\vartheta_{m}\left(Z_{0}\right)
$$

We may assume that the curves $\vartheta_{m^{\prime}}\left(Z_{s}\right)$ and $\vartheta_{m^{\prime \prime}}\left(Z_{s}\right)$ are analytic (in $s$ ) in what follows.

When considering $X_{2}^{s}=\sum_{m \in \mathcal{M}\left(Z_{s}\right)} \xi_{m}^{s}$, note that the subscript $m$ might depend on $s$ in the limit. However, we may assume that $\mu\left(Z_{0}\right) \leq \mu\left(Z_{s}\right)$ for all $s$ near 0 . If $\mu\left(Z_{0}\right)<\mu\left(Z_{s}\right)$, we view $W_{m}\left(Z_{0}\right)$ as the sum of its refined invariant subspaces, which we now define.

For sufficiently small positive values of $s, j\left(Z_{s}\right)$ has invariant subspaces $W_{m^{\prime}}\left(Z_{s}\right)$ and $W_{m^{\prime \prime}}\left(Z_{s}\right)$, respectively. By $\left[1\right.$, Theorem 4.16], since $j\left(Z_{s}\right) \rightarrow$ $j\left(Z_{0}\right)$ and skew-symmetry holds, we may define

$$
W^{\prime}\left(Z_{0}\right)=\lim _{s \rightarrow 0} W_{m^{\prime}}\left(Z_{s}\right) \quad \text { and } \quad W^{\prime \prime}\left(Z_{0}\right)=\lim _{s \rightarrow 0} W_{m^{\prime \prime}}\left(Z_{s}\right) .
$$

Note that since $W_{m^{\prime}}\left(Z_{s}\right)$ and $W_{m^{\prime \prime}}\left(Z_{s}\right)$ are orthogonal, invariant subspaces of $j\left(Z_{s}\right)$ for all $s$, their limit spaces $W^{\prime}\left(Z_{0}\right)$ and $W^{\prime \prime}\left(Z_{0}\right)$ are orthogonal, invariant subspaces of $j\left(Z_{0}\right)$, and

$$
W_{m}\left(Z_{0}\right)=W^{\prime}\left(Z_{0}\right) \oplus W^{\prime \prime}\left(Z_{0}\right)
$$

We refer to $W^{\prime}\left(Z_{0}\right), W^{\prime \prime}\left(Z_{0}\right)$ as refined invariant subspaces of $j\left(Z_{0}\right)$.

By passing to refined invariant subspaces when necessary, we deduce that all of the derivatives exist below, even in the case that $Z_{0} \notin \mathcal{U}$, and that the 
limiting values are as given. Another technical detail is the possibility that $\lim _{s \rightarrow 0} \vartheta_{m}\left(Z_{s}\right)=0$ for some $m$ such that $\xi_{m}^{s} \not \equiv 0$. To account for this, we write

$$
\mathcal{M}=N \cup M
$$

where $N$ is defined as the subset of $\mathcal{M}$ such that, for $n \in N, \lim _{s \rightarrow 0} \vartheta_{n}\left(Z_{s}\right)=$ 0 and $\vartheta_{n}\left(Z_{s}\right)>0$ for all $s \neq 0$. Define $M$ as the subset of $\mathcal{M}$ such that, for $m \in M, \lim _{s \rightarrow 0} \vartheta_{m}\left(Z_{s}\right) \neq 0$. Thus $X_{2}^{s}=\sum_{m \in M} \xi_{m}^{s}+\sum_{n \in N} \xi_{n}^{s}$. Note that if $\vartheta_{1}\left(Z_{s}\right)=0$ for all $s$, then $\xi_{1}^{s}=0$ for all $s$, and the vector component corresponding to $\vartheta_{1}\left(Z_{s}\right)$ is included in $X_{1}^{s}$ and not in $X_{2}^{s}$. Because of this possibility, the notations $X_{1}^{0}$ and $X_{2}^{0}$ are ambiguous.

We use the convention that $a_{2}(0)=X_{1}+X_{2}+Z_{0}$ as in Notation 2.9, while $X_{i}^{0}=\lim _{s \rightarrow 0} X_{i}^{s}, i=1,2$ and $Z_{0}^{0}=\lim _{s \rightarrow 0} Z_{0}^{s}$. Thus $Z_{0}^{0}=Z_{0}$ but

$$
X_{1}=X_{1}^{0}+\sum_{n \in N} \xi_{n}^{0}
$$

and

$$
X_{2}=X_{2}^{0}-\sum_{n \in N} \xi_{n}^{0}=\sum_{m \in M} \xi_{m}^{0}
$$

where we define $\xi_{m}^{0}=\lim _{s \rightarrow 0} \xi_{m}^{s}, J_{s}=j\left(Z_{0}^{s}\right)$ and $\vartheta_{m}^{s}=\vartheta_{m}\left(Z_{0}^{s}\right)$.

Let $\alpha(s)=\left(\tilde{p} \exp \left(a_{1}(s)\right), a_{2}(s)\right)$ be a curve in $\tilde{\mathfrak{X}}$ with $a_{1}(0)=0, a_{2}(0)=$ $v=X_{1}+X_{2}+Z_{0}$ and $v_{p} \in F\left(\tau, c_{\gamma}\right)$. Now $a_{2}(s)=X_{1}^{s}+X_{2}^{s}+Z_{0}^{s}$ where $Z_{0}^{s} \in \mathfrak{z}, \quad X_{1}^{s}+X_{2}^{s} \perp \mathfrak{z}, \quad X_{1}^{s} \in \operatorname{ker} j\left(Z_{0}^{s}\right)$ and $X_{2}^{s} \perp \operatorname{ker} j\left(Z_{0}^{s}\right)$. Also, $X_{2}^{s}=$ $\sum_{n \in N} \xi_{n}^{s}+\sum_{m \in M} \xi_{m}^{s}$, where $\xi_{m}^{s} \in W_{m}\left(Z_{0}^{s}\right)$ and $\xi_{n}^{s} \in W_{n}\left(Z_{0}^{s}\right)$ as above. Define $\Xi_{m}^{s}=J_{s} \xi_{m}^{s} / \vartheta_{m}^{s}$, for $m \in \mathcal{M}\left(Z_{0}^{s}\right)$.

Let $\exp \left(X^{s}(r)+Z^{s}(r)\right)$ be the geodesic through $e$ with initial velocity $a_{2}(s)$. Eberlein [12] first explicitly solved the geodesic equations, so that the values for $X^{s}(r)$ and $Z^{s}(r)$ are as follows.

$$
\begin{aligned}
X^{s}(r)= & r X_{1}^{s}+\left(e^{r J_{s}}-\mathrm{Id}\right)\left(J_{s}^{-1} X_{2}^{s}\right) \\
= & r X_{1}^{s}+\sum_{m \in M} \frac{1-\cos \left(r \vartheta_{m}^{s}\right)}{\vartheta_{m}^{s}} \Xi_{m}^{s}+\sum_{m \in M} \frac{\sin \left(r \vartheta_{m}^{s}\right)}{\vartheta_{m}^{s}} \xi_{m}^{s} \\
& +\sum_{n \in N} \frac{1-\cos \left(r \vartheta_{n}^{s}\right)}{\vartheta_{n}^{s}} \Xi_{n}^{s}+\sum_{n \in N} \frac{\sin \left(r \vartheta_{n}^{s}\right)}{\vartheta_{n}^{s}} \xi_{n}^{s}
\end{aligned}
$$




$$
\begin{aligned}
X^{\prime s}(r)= & X_{1}^{s}+e^{r J_{s}} X_{2}^{s} \\
= & X_{1}^{s}+\sum_{m \in M} \sin \left(r \vartheta_{m}^{s}\right) \Xi_{m}^{s}+\sum_{m \in M} \cos \left(r \vartheta_{m}^{s}\right) \xi_{m}^{s} \\
& +\sum_{n \in N} \frac{\sin \left(r \vartheta_{n}^{s}\right)}{\vartheta_{n}^{s}}\left(j\left(Z_{0}^{s}\right) \xi_{n}^{s}\right)+\sum_{n \in N} \cos \left(r \vartheta_{n}^{s}\right) \xi_{n}^{s} \\
Z^{s}(\tau)= & \int_{0}^{\tau}\left(Z_{0}^{s}+\frac{1}{2}\left[X^{s}(r), X^{\prime s}(r)\right]\right) d r .
\end{aligned}
$$

Taking the derivative with respect to $s$ of $X^{s}(r)$ and evaluating at $s=0$, we have

$$
\begin{aligned}
\dot{X}^{0}(r)= & r \dot{X}_{1}^{0}+\sum_{m \in M} \dot{\vartheta}_{m}^{0}\left(\frac{r \vartheta_{m}^{0} \sin \left(r \vartheta_{m}^{0}\right)+\cos \left(r \vartheta_{m}^{0}\right)-1}{\vartheta_{m}^{0}{ }^{2}}\right) \Xi_{m}^{0} \\
& +\sum_{m \in M} \dot{\vartheta}_{m}^{0}\left(\frac{r \vartheta_{m}^{0} \cos \left(r \vartheta_{m}^{0}\right)-\sin \left(r \vartheta_{m}^{0}\right)}{\vartheta_{m}^{0}{ }^{2}}\right) \xi_{m}^{0}+\sum_{m \in M} \frac{1-\cos \left(r \vartheta_{m}^{0}\right)}{\vartheta_{m}^{0}} \dot{\Xi}_{m}^{0} \\
& +\sum_{m \in M} \frac{\sin \left(r \vartheta_{m}^{0}\right)}{\vartheta_{m}^{0}} \dot{\xi}_{m}^{0}+\sum_{n \in N} \dot{\vartheta}_{n}^{0}\left(\frac{r \vartheta_{n}^{0} \sin \left(r \vartheta_{n}^{0}\right)+\cos \left(r \vartheta_{n}^{0}\right)-1}{\vartheta_{n}^{0}}\right) \Xi_{n}^{0} \\
& +\sum_{n \in N} \dot{\vartheta}_{n}^{0}\left(\frac{r \vartheta_{n}^{0} \cos \left(r \vartheta_{n}^{0}\right)-\sin \left(r \vartheta_{n}^{0}\right)}{\vartheta_{n}^{0}}\right) \xi_{n}^{0}+\sum_{n \in N} \frac{1-\cos \left(r \vartheta_{n}^{0}\right)}{\vartheta_{n}^{0}} \dot{\Xi}_{n}^{0} \\
& +\sum_{n \in N} \frac{\sin \left(r \vartheta_{n}^{0}\right)}{\vartheta_{n}^{0}} \dot{\xi}_{n}^{0} .
\end{aligned}
$$

Note that for $m \in M$, the values above are clearly well defined, since all of the values are analytic in $s$. For $n \in N$, observe that $\left|\Xi_{n}^{s}\right|=\left|\frac{1}{\vartheta_{n}^{s}} J_{s} \xi_{n}^{s}\right|=$ $\left|\xi_{n}^{s}\right|$ by using the definition of $\xi_{n}^{s}(2.15)$, so $\Xi_{n}^{s}=\frac{1}{\vartheta_{n}^{s}} J_{s} \xi_{n}^{s}$ is likewise well defined with continuous derivative in $s$. But for $n \in N$ we now abuse notation slightly. For example, for $n \in N$, $\sin \left(r \vartheta_{n}^{0}\right) / \vartheta_{n}^{0}$ is technically not defined, while $\lim _{s \rightarrow 0} \sin \left(r \vartheta_{n}^{s}\right) / \vartheta_{n}^{s}$ exists and is well defined. We use the convention that for $n \in N$, when necessary, seemingly undefined values are defined as the limit as $s \rightarrow 0^{+}$of nearby values. With this established, for $n \in N$

$$
\begin{aligned}
& \left(\frac{r \vartheta_{n}^{0} \sin \left(r \vartheta_{n}^{0}\right)+\cos \left(r \vartheta_{n}^{0}\right)-1}{\vartheta_{n}^{0}}\right)=\frac{r^{2}}{2}, \quad\left(\frac{r \vartheta_{n}^{0} \cos \left(r \vartheta_{n}^{0}\right)-\sin \left(r \vartheta_{n}^{0}\right)}{\vartheta_{n}^{0}{ }^{2}}\right)=0 \\
& \dot{\vartheta}_{n}^{0} \Xi_{n}^{0}=\frac{\dot{\vartheta}_{n}^{0} J_{0} \xi_{n}^{0}}{\vartheta_{n}^{0}}=\left.\frac{d}{d s}\right|_{\left.\right|_{0}}\left(J_{s} \xi_{n}^{s}\right), \quad \frac{1-\cos \left(r \vartheta_{n}^{0}\right)}{\vartheta_{n}^{0}}=0, \quad \frac{\sin \left(r \vartheta_{n}^{0}\right)}{\vartheta_{n}^{0}}=r .
\end{aligned}
$$


We now have

$$
\begin{aligned}
X^{0}(r)= & r\left(X_{1}^{0}+\sum_{n \in N} \xi_{n}^{0}\right)+\sum_{m \in M} \frac{1-\cos \left(r \vartheta_{m}^{0}\right)}{\vartheta_{m}^{0}} \Xi_{m}^{0}+\sum_{m \in M} \frac{\sin \left(r \vartheta_{m}^{0}\right)}{\vartheta_{m}^{0}} \xi_{m}^{0}, \\
X^{\prime}(r)= & \left(X_{1}^{0}+\sum_{n \in N} \xi_{n}^{0}\right)+\sum_{m \in M} \sin \left(r \vartheta_{m}^{0}\right) \Xi_{m}^{0}+\sum_{m \in M} \cos \left(r \vartheta_{m}^{0}\right) \xi_{m}^{0}, \\
\dot{X}^{0}(r)= & r\left(\dot{X}_{1}^{0}+\sum_{n \in N} \dot{\xi}_{n}^{0}\right)+\sum_{m \in M} \dot{\vartheta}_{m}^{0}\left(\frac{r \vartheta_{m}^{0} \sin \left(r \vartheta_{m}^{0}\right)+\cos \left(r \vartheta_{m}^{0}\right)-1}{\vartheta_{m}^{0}{ }^{2}}\right) \Xi_{m}^{0} \\
& +\sum_{m \in M} \dot{\vartheta}_{m}^{0}\left(\frac{r \vartheta_{m}^{0} \cos \left(r \vartheta_{m}^{0}\right)-\sin \left(r \vartheta_{m}^{0}\right)}{\vartheta_{m}^{0}{ }^{2}}\right) \xi_{m}^{0}+\sum_{m \in M} \frac{1-\cos \left(r \vartheta_{m}^{0}\right)}{\vartheta_{m}^{0}} \dot{\Xi}_{m}^{0} \\
& +\sum_{m \in M} \frac{\sin \left(r \vartheta_{m}^{0}\right)}{\vartheta_{m}^{0}} \dot{\xi}_{m}^{0}+\left.\sum_{n \in N} \frac{r^{2}}{2} \frac{d}{d s}\right|_{\left.\right|_{0}}\left(J_{s} \xi_{n}^{s}\right) .
\end{aligned}
$$

A similar analysis yields

$$
\begin{aligned}
\dot{X}^{\prime 0}(r)= & \left(\dot{X}_{1}^{0}+\sum_{n \in N} \dot{\xi}_{n}^{0}\right)+\sum_{m \in M} r \dot{\vartheta}_{m}^{0} \cos \left(r \vartheta_{m}^{0}\right) \Xi_{m}^{0}-\sum_{m \in M} r \dot{\vartheta}_{m}^{0} \sin \left(r \vartheta_{m}^{0}\right) \xi_{m}^{0} \\
& +\sum_{m \in M} \sin \left(r \vartheta_{m}^{0}\right) \dot{\Xi}_{m}^{0}+\sum_{m \in M} \cos \left(r \vartheta_{m}^{0}\right) \dot{\xi}_{m}^{0}+\left.\sum_{n \in N} r \frac{d}{d s}\right|_{\left.\right|_{0}}\left(J_{s} \xi_{n}^{s}\right) .
\end{aligned}
$$

If we let $r=\tau$ in the above, then by condition (d) of Theorem 2.12, if $\xi_{m}^{0} \neq 0$ then $\tau \vartheta_{m}^{0} \in 2 \pi \mathbb{Z}^{+}$. Also note that $\xi_{m}^{0} \neq 0$ if and only if $\Xi_{m}^{0} \neq 0$. However, if $\xi_{m}^{0}=0$ but $\dot{\xi}_{m}^{0} \neq 0$, we cannot assume $\tau \vartheta_{m}^{0} \in 2 \pi \mathbb{Z}^{+}$. We thus divide the set $M$ into two disjoint subsets.

$$
M^{\prime}=\left\{m \in M: \xi_{m}^{0} \neq 0\right\} \quad \text { and } \quad M^{\prime \prime}=\left\{m \in M: \xi_{m}^{0}=0\right\}
$$

We then have

$$
\begin{aligned}
\dot{X}^{0}(\tau)= & \tau\left(\dot{X}_{1}^{0}+\sum_{n \in N} \dot{\xi}_{n}^{0}\right)+\sum_{m \in M^{\prime}}\left(\frac{\tau \dot{\vartheta}_{m}^{0}}{\vartheta_{m}^{0}}\right) \xi_{m}^{0}+\left.\sum_{n \in N} \frac{\tau^{2}}{2} \frac{d}{d s}\right|_{0}\left(J_{s} \xi_{n}^{s}\right) \\
& +\sum_{m \in M^{\prime \prime}} \frac{1-\cos \left(\tau \vartheta_{m}^{0}\right)}{\vartheta_{m}^{0}} \dot{\Xi}_{m}^{0}+\sum_{m \in M^{\prime \prime}} \frac{\sin \left(\tau \vartheta_{m}^{0}\right)}{\vartheta_{m}^{0}} \dot{\xi}_{m}^{0}
\end{aligned}
$$


A similar analysis yields

$$
\begin{aligned}
\dot{X}^{\prime 0}(\tau)= & \left(\dot{X}_{1}^{0}+\sum_{n \in N} \dot{\xi}_{n}^{0}+\sum_{m \in M^{\prime}} \dot{\xi}_{m}^{0}\right)+\sum_{m \in M^{\prime}} \tau \dot{\vartheta}_{m}^{0} \Xi_{m}^{0}+\sum_{n \in N} \tau \frac{d}{\left.d s\right|_{0}}\left(J_{s} \xi_{n}^{s}\right) \\
& +\sum_{m \in M^{\prime \prime}} \sin \left(\tau \vartheta_{m}^{0}\right) \dot{\Xi}_{m}^{0}+\sum_{m \in M^{\prime \prime}} \cos \left(\tau \vartheta_{m}^{0}\right) \dot{\xi}_{m}^{0}
\end{aligned}
$$

which implies

$$
\begin{aligned}
-\dot{X}^{\prime 0}(\tau)+\dot{X}_{0}^{0}= & -\sum_{m \in M^{\prime}} \tau \dot{\vartheta}_{m}^{0} \Xi_{m}^{0}-\sum_{n \in N} \tau \frac{d}{\left.d s\right|_{0}}\left(J_{s} \xi_{n}^{s}\right) \\
& -\sum_{m \in M^{\prime \prime}} \sin \left(\tau \vartheta_{m}^{0}\right) \dot{\Xi}_{m}^{0}+\sum_{m \in M^{\prime \prime}}\left(1-\cos \left(\tau \vartheta_{m}^{0}\right)\right) \dot{\xi}_{m}^{0}
\end{aligned}
$$

Now recall that

$$
\dot{Z}^{0}(\tau)=\left.\frac{d}{d s}\right|_{0} \int_{0}^{\tau}\left(Z_{0}^{s}+\frac{1}{2}\left[X^{s}(r), X^{\prime} s(r)\right]\right) d r .
$$

Since everything in sight is analytic in both $s$ and $r$ and bounded with bounded derivative in $s$ near 0 , and since we are integrating over a finite, closed interval, we can use uniform convergence to bring the derivative $\frac{d}{d s}$ under the integral sign. Using the linearity of the Lie bracket in each component and the product rule, we thus have

$$
\left.\frac{d}{d s}\right|_{0} Z^{s}(\tau)=\int_{0}^{\tau}\left(\dot{Z}_{0}^{0}+\frac{1}{2}\left[\dot{X}^{0}(r), X^{\prime 0}(r)\right]+\frac{1}{2}\left[X^{0}(r), \dot{X}^{\prime 0}(r)\right]\right) d r .
$$

After substituting in the above values of $X^{0}(r), X^{\prime 0}(r), \dot{X}^{0}(r)$ and $\dot{X}^{\prime} 0(r)$, using the fact that $X_{1}=\frac{1}{\tau} V$, integrating, and using the fact that for $m \in M^{\prime}, \tau \vartheta_{m}^{0} \in 2 \pi \mathbb{Z}^{+}$, we obtain

$$
\begin{aligned}
\dot{Z}^{0}(\tau)= & \tau \dot{Z}_{0}^{0}+\left[V, \int_{0}^{\tau} \frac{1}{2 \tau}\left(r \dot{X}^{\prime} 0(r)-\dot{X}^{0}(r)\right) d r\right] \\
& +\sum_{\substack{m \in M, h \in M^{\prime} \\
\vartheta_{m}^{0}=\vartheta_{h}^{0}}} \frac{\tau}{2 \vartheta_{m}^{0}}\left(\left[\dot{\xi}_{m}^{0}, \Xi_{h}^{0}\right]+\left[\xi_{h}^{0}, \dot{\Xi}_{m}^{0}\right]\right) \\
& -\sum_{m \in M^{\prime}} \frac{\tau}{\vartheta_{m}^{0}}\left[\dot{X}_{1}^{0}+\sum_{n \in N} \dot{\xi}_{n}^{0}, \Xi_{m}^{0}\right] \\
& +\sum_{m \in M^{\prime}, n \in N} \frac{\tau^{2}}{2 \vartheta_{m}^{0}}\left[\Xi_{m}^{0},\left.\frac{d}{d s}\right|_{0}\left(J_{s} \xi_{n}^{s}\right)\right]
\end{aligned}
$$


(2.21c)

$$
\begin{aligned}
& -\sum_{m \in M^{\prime}, n \in N} \frac{\tau}{\vartheta_{m}^{0}{ }^{2}}\left[\xi_{m}^{0},\left.\frac{d}{d s}\right|_{0}\left(J_{s} \xi_{n}^{s}\right)\right] \\
& +\sum_{\substack{m, h \in M^{\prime} \\
\vartheta_{m}^{0} \neq \vartheta_{h}^{0}}} \frac{\tau\left(\dot{\vartheta}_{h}^{0} \vartheta_{m}^{0}-\vartheta_{h}^{0} \dot{\vartheta}_{m}^{0}\right)}{\vartheta_{h}^{0}\left(\vartheta_{m}^{0}{ }^{2}-\vartheta_{h}^{0^{2}}\right)}\left[\Xi_{m}^{0}, \xi_{h}^{0}\right] \\
& +\sum_{\substack{m, h \in M^{\prime} \\
\vartheta_{m}^{0}=\vartheta_{h}^{0}}} \frac{\tau\left(3 \dot{\vartheta}_{h}^{0}+\dot{\vartheta}_{m}^{0}\right)}{4 \vartheta_{m}^{0}{ }^{2}}\left[\Xi_{m}^{0}, \xi_{h}^{0}\right] \\
& +\sum_{\substack{m, h \in M^{\prime} \\
\vartheta_{m}^{0}=\vartheta_{h}^{0}}} \frac{\tau^{2}\left(\dot{\vartheta}_{m}^{0}-\dot{\vartheta}_{h}^{0}\right)}{8 \vartheta_{m}^{0}}\left(\left[\Xi_{m}^{0}, \Xi_{h}^{0}\right]+\left[\xi_{m}^{0}, \xi_{h}^{0}\right]\right) \\
& +\sum_{\substack{m \in M^{\prime \prime}, h \in M^{\prime} \\
\vartheta_{m}^{0} \neq \vartheta_{h}^{0}}} \frac{\vartheta_{h}^{0}\left(1-\cos \left(\tau \vartheta_{m}^{0}\right)\right)}{\vartheta_{m}^{0}\left(\vartheta_{m}^{0}-\vartheta_{h}^{0}\right)}\left[\dot{\Xi}_{m}^{0}, \Xi_{h}^{0}\right] \\
& -\sum_{\substack{m \in M^{\prime \prime}, h \in M^{\prime} \\
\vartheta_{m}^{0} \neq \vartheta_{h}^{0}}} \frac{\sin \left(\tau \vartheta_{m}^{0}\right)}{\vartheta_{m}^{0}-\vartheta_{h}^{0^{2}}}\left[\dot{\Xi}_{m}^{0}, \xi_{h}^{0}\right] \\
& +\sum_{\substack{m \in M^{\prime \prime}, h \in M^{\prime} \\
\vartheta_{m}^{0} \neq \vartheta_{h}^{0}}} \frac{\vartheta_{h}^{0} \sin \left(\tau \vartheta_{m}^{0}\right)}{\vartheta_{m}^{0}\left(\vartheta_{m}^{0}{ }^{2}-\vartheta_{h}^{0^{2}}\right)}\left[\dot{\xi}_{m}^{0}, \Xi_{h}^{0}\right] \\
& +\sum_{\substack{m \in M^{\prime \prime}, h \in M^{\prime} \\
\vartheta_{m}^{0} \neq \vartheta_{h}^{0}}} \frac{1-\cos \left(\tau \vartheta_{m}^{0}\right)}{\vartheta_{m}^{0}-\vartheta_{h}^{0}}\left[\dot{\xi}_{m}^{0}, \xi_{h}^{0}\right] .
\end{aligned}
$$

Similarly

$$
\begin{aligned}
\int_{0}^{\tau} & \frac{1}{2 \tau}\left(r \dot{X}^{\prime 0}(r)-\dot{X}^{0}(r)\right) d r \\
= & \sum_{m \in M^{\prime}} \frac{2 \dot{\vartheta}_{m}^{0}}{\vartheta_{m}^{0} \Xi_{m}^{0}}+\sum_{m \in M^{\prime}} \frac{\tau \dot{\vartheta}_{m}^{0}}{2 \vartheta_{m}^{0}} \xi_{m}^{0}-\sum_{m \in M^{\prime}} \frac{1}{\vartheta_{m}^{0}} \dot{\Xi}_{m}^{0}+\left.\sum_{n \in N} \frac{\tau^{2}}{12} \frac{d}{d s}\right|_{0}\left(J_{s} \xi_{n}^{s}\right) \\
& +\sum_{m \in M^{\prime \prime}} \frac{2 \sin \left(\tau \vartheta_{m}^{0}\right)-\tau \vartheta_{m}^{0}\left(\cos \left(\tau \vartheta_{m}^{0}\right)+1\right)}{2 \tau \vartheta_{m}^{0}{ }^{2}} \dot{\Xi}_{m}^{0} \\
& +\sum_{m \in M^{\prime \prime}} \frac{2\left(\cos \left(\tau \vartheta_{m}^{0}\right)-1\right)+\tau \vartheta_{m}^{0} \sin \left(\tau \vartheta_{m}^{0}\right)}{2 \tau \vartheta_{m}^{0}{ }^{2}} \dot{\xi}_{m}^{0}
\end{aligned}
$$


Finally, we require the canonical symplectic form on $T_{v_{\tilde{p}}} T G$ viewed as $T_{p} G \times T_{v} \mathfrak{g}$. Recall that we use the canonical form on the dual bundle together with the inner product and the musical isomorphisms to obtain the canonical symplectic form on $T_{v_{\tilde{p}}} T G$.

Theorem 2.20 [13]. With notation as above, for a Riemannian two-step nilmanifold, the canonical symplectic form on $T_{v_{\tilde{p}}} T G=T_{p} G \times T_{v} \mathfrak{g}$ is

$$
\omega_{v_{\tilde{p}}}\left(\left(A_{1}, B_{1}\right),\left(A_{2}, B_{2}\right)\right)=\left\langle A_{1}, B_{2}\right\rangle-\left\langle A_{2}, B_{1}\right\rangle+\left\langle v,\left[A_{1}, A_{2}\right]\right\rangle,
$$

where $A_{1}, A_{2} \in T_{p} G=L_{p *} \mathfrak{g}$ and $B_{1}, B_{2} \in T_{v} \mathfrak{g}$.

\section{The DG-multiplier in the wave invariants}

We now use (2.18) and (2.21) to determine the Poincaré map, and in some cases the DG-multiplier, for the periods associated to an arbitrary element $\gamma \in \Gamma$ for an arbitrary two-step nilmanifold $(\Gamma \backslash G, g)$.

We use Theorem 2.11 and Remark 2.13 to divide our computations into several cases.

Case I. $\gamma=\exp \left(V+Z_{V}\right)$ and $\tau=|V|$ (i.e., $Z_{V}^{\perp}=0$ )

Case II. $\gamma=\exp (Z)$ and $\tau=|Z|$

Case III. $\gamma=\exp (Z)$ and $\tau<|Z|$

Case IV. $\gamma=\exp \left(V+Z_{V}+Z_{V}^{\perp}\right), Z_{V}^{\perp} \neq 0$ and $\tau=\left|V+Z_{V}^{\perp}\right|$

Case V. $\gamma=\exp \left(V+Z_{V}+Z_{V}^{\perp}\right), Z_{V}^{\perp} \neq 0$ and $\tau<\left|V+Z_{V}^{\perp}\right|$.

In Cases I, II and IV we are also able to obtain a reasonable, geometric formula for the DG-multiplier. In Cases III and V, the expression of the Poincaré map is sufficiently prohibitive. We are able to compute the DGmultiplier for Case III and V only in special cases or specific examples. See Section 4 for the special case of a one-dimensional center/Heisenberg manifold, and a specific five-dimensional example.

Recall that we seek to calculate $d \mu$ from (2.3) and Lemma 2.2. For this, we must calculate $T=I-\tilde{\Phi}_{\tau *_{v \tilde{p}}}$ on a basis of $T \mathfrak{X}$ normal to $T_{v_{\tilde{p}}} \tilde{F}(\tau, \gamma)$. For $x \in G$, define $C(x, G)$ as the centralizer of $x$ in $G$. For $X \in \mathfrak{g}$, define $C(X, \mathfrak{g})$ as the centralizer of $X$ in $\mathfrak{g}$. 
We continue the notation of Section 2.

Case I: Poincaré Map. $\gamma=\exp \left(V+Z_{V}\right)$ and $\tau=|V|$ (i.e., $\left.Z_{V}^{\perp}=0\right)$.

Let $\bar{V}=V / \tau=V /|V|$. It follows from Theorem 2.12 that $\tilde{F}(\tau, \gamma)=$ $C(\gamma, G) \tilde{q} \times\{\bar{V}\}$ where $\tilde{q} \exp (V) \tilde{q}^{-1}=\gamma$. Let $v_{\tilde{p}} \in \tilde{F}(\tau, \gamma)$, so $v_{\tilde{p}}=(\tilde{p}, \bar{V})$ and $\tilde{p}=x \tilde{q}$ where $x \in C(\gamma, G)$. So that $\tilde{p} \exp (V)=\gamma \tilde{p}$. Then

$$
T_{v_{\tilde{p}}} \tilde{F}(\tau, \gamma)=C(\log \gamma, \mathfrak{g}) \times\{0\}
$$

As $\tilde{\Phi}_{\tau *_{\tilde{p}}}=\mathrm{Id}$ when restricted to $C(\log \gamma, \mathfrak{g}) \times\{0\}$, we must calculate (1) $\tilde{\Phi}_{\tau *_{\tilde{p}}}(U, 0)$ where $U \perp C(\log \gamma, \mathfrak{g})$, (2) $\tilde{\Phi}_{\tau *_{\tilde{p}}}(0, U)$ where $U \perp V$ and $U \in \mathfrak{v}$ and $(3) \tilde{\Phi}_{\tau *_{\tilde{p}}}(0, \zeta)$ where $\zeta \in \mathfrak{z}$.

For $(1)$, we let $\alpha(s)=(\tilde{p} \exp (s U), \bar{V})$, so that $a_{1}(s)=s U$ and $a_{2}(s)=\bar{V}$. Thus $Z_{0}^{s}=0, X_{2}^{s}=0$ and $X_{1}^{s}=\bar{V}$. Using $(2.21)$ we see that $\dot{X}^{0}(\tau)=0$, $-\dot{X}^{\prime} 0(\tau)+\dot{X}_{0}^{0}=0$ and $\dot{Z}^{0}(\tau)=0$, thus by $(2.18)$

$$
\left(\mathrm{Id}-\tilde{\Phi}_{\tau *_{\tilde{p}}}\right)(U, 0)=([V, U], 0) .
$$

For $(2)$, we let $\alpha(s)=(\tilde{p}, \cos s \bar{V}+\sin s U)$, so that $a_{1}(s)=e, a_{2}(s)=$ $\cos s \bar{V}+\sin s U, \dot{a}_{1}(0)=0$ and $\dot{a}_{2}(0)=U$. Here $Z_{0}^{s}=0$ so $X_{2}^{s}=0$ and $X_{1}^{s}=$ $\cos s \bar{V}+\sin s U$. By $(2.21), \dot{X}^{0}(\tau)=\tau U,-\dot{X}^{\prime}(\tau)+\dot{X}_{0}^{0}=0$ and $\dot{Z}^{0}(\tau)=0$. Thus by (2.18)

$$
\left(\mathrm{Id}-\tilde{\Phi}_{\tau *_{\tilde{p}}}\right)(0, U)=\left(-\tau U+\frac{\tau}{2}[V, U], 0\right)
$$

For $(3)$, we let $\alpha(s)=(\tilde{p}, \cos s \bar{V}+\sin s \zeta)$, so that $a_{1}(s)=e, a_{2}(s)=$ $\cos s \bar{V}+\sin s \zeta, \dot{a}_{1}(0)=0$ and $\dot{a}_{2}(0)=\zeta$. Here $Z_{0}^{s}=\sin s \zeta$ and $\dot{Z}_{0}^{0}=\zeta$. Let $\bar{V}=X_{1}+\sum_{m \in \mathcal{M}} \xi_{m}$, where $X_{1} \in \operatorname{ker} j(\zeta)$ and $\xi_{m} \in W_{m}(\zeta)$ for $m \in$ $\mathcal{M}(\zeta)$. Thus $X_{0}^{s}=\cos s X_{1}+\sum_{m \in \mathcal{M}} \cos s \xi_{m}, X_{1}^{s}=\cos s X_{1}$ and $\dot{X}_{1}^{0}=0$. Note that $\xi_{m}^{s}=\cos s \xi_{m}$, so that $\xi_{m}^{0}=\xi_{m}, \dot{\xi}_{m}^{0}=0, \Xi_{m}^{0}=j(\zeta) \xi_{m} / \theta_{m}(\zeta)$ and $\dot{\Xi}_{m}^{0}=0$. Also, $\vartheta_{m}^{s}=\sin s \vartheta_{m}(\zeta)$, so that $\vartheta_{m}^{0}=0, \dot{\vartheta}_{m}^{0}=\vartheta_{m}, N=\mathcal{M}$ and $M=\emptyset$. Finally, $\left.\frac{d}{d s}\right|_{0}\left(J_{s} \xi_{n}^{s}\right)=j(\zeta) \xi_{n}$ for $n \in N$. Plugging these values into (2.21), we have $\dot{X}^{0}(\tau)=\frac{\tau^{2}}{2} j(\zeta) \bar{V},-\dot{X}^{\prime 0}(\tau)+\dot{X}_{0}^{0}=-\tau j(\zeta) \bar{V}$ and $\dot{Z}^{0}(\tau)=$ $\tau \zeta+\frac{\tau}{12}[V, j(\zeta) V]$. Thus by $(2.18)$

$$
\left(\operatorname{Id}-\tilde{\Phi}_{\tau *_{\tilde{p}}}\right)(0, \zeta)=\left(-\frac{\tau}{2} j(\zeta) V-\tau \zeta+\frac{\tau}{6}[V, j(\zeta) V],-j(\zeta) V\right) .
$$

Case I: DG-Multiplier. We must now wisely choose bases $\mathcal{E}, \mathcal{V}$ and $\mathcal{F}$ so that $d \mu$ can be expressed in terms of $\tau, \gamma$ and $v_{\tilde{p}}$. We continue the notation of Lemma 2.2. 
Let $\left\{\bar{V}, Y_{1}, \ldots, Y_{a}, \zeta_{1}, \ldots, \zeta_{b}, \zeta_{b+1}, \ldots, \zeta_{c}\right\}$ be an orthonormal basis of $C(\log \gamma, \mathfrak{g})$ such that $\mathfrak{z}=\operatorname{span}_{\mathbb{R}}\left\{\zeta_{1}, \ldots, \zeta_{c}\right\},[V, \mathfrak{g}]=\operatorname{span}_{\mathbb{R}}\left\{\zeta_{1}, \ldots, \zeta_{b}\right\}, \zeta_{j} \perp$ $[V, \mathfrak{g}]$ for $j=b+1, \ldots, c$ and $Y_{i} \perp \mathfrak{z}, i=1, \ldots, a$. Let $C(\log \gamma, \mathfrak{g})^{\perp}$ be the orthogonal complement of $C(\log \gamma, \mathfrak{g})$ in $\mathfrak{g}$. Let $\left\{Y_{1}^{\perp}, \ldots, Y_{b}^{\perp}\right\}$ be an orthonormal basis of $C(\log \gamma, \mathfrak{g})^{\perp}$. The set $\left\{\left[V, Y_{1}^{\perp}\right], \ldots,\left[V, Y_{b}^{\perp}\right]\right\}$ forms a basis of $[V, \mathfrak{g}]$, so the dimension of $[V, \mathfrak{g}]$ is equal to the dimension of $C(\log \gamma, \mathfrak{g})^{\perp}$.

We set

$$
\mathcal{E}=\left\{(\bar{V}, 0),\left(Y_{1}, 0\right), \ldots,\left(Y_{a}, 0\right),\left(\zeta_{1}, 0\right), \ldots,\left(\zeta_{c}, 0\right),(0, \bar{V})\right\}
$$

Then using Theorem 2.20 we compute

$$
\mathcal{F}=\left\{(0, \bar{V}),\left(0, Y_{1}\right), \ldots,\left(0, Y_{a}\right),\left(0, \zeta_{1}\right), \ldots,\left(0, \zeta_{c}\right),(-\bar{V}, 0)\right\}
$$

We set

$$
\begin{aligned}
\mathcal{V}= & \left\{\left(Y_{1}^{\perp}, 0\right), \ldots,\left(Y_{b}^{\perp}, 0\right),\left(0, Y_{1}^{\perp}\right), \ldots,\left(0, Y_{b}^{\perp}\right),\left(0, Y_{1}\right), \ldots,\left(0, Y_{a}\right)\right. \\
& \left.\left(0, \zeta_{1}\right), \ldots,\left(0, \zeta_{c}\right)\right\}
\end{aligned}
$$

We must calculate $T \mathcal{V} \wedge \mathcal{F}$ as a multiple of $\mathcal{V} \wedge \mathcal{E}$, up to sign. Now $T \mathcal{V} \wedge \mathcal{F}$ equals

$$
\begin{aligned}
& T\left(Y_{1}^{\perp}, 0\right) \wedge \cdots \wedge T\left(Y_{b}^{\perp}, 0\right) \wedge T\left(0, Y_{1}^{\perp}\right) \wedge \cdots \wedge T\left(0, Y_{b}^{\perp}\right) \\
& \wedge T\left(0, Y_{1}\right) \wedge \cdots \wedge T\left(0, Y_{a}\right) \\
& \wedge T\left(0, \zeta_{1}\right) \wedge \cdots \wedge T\left(0, \zeta_{c}\right) \wedge(0, \bar{V}) \wedge\left(0, Y_{1}\right) \wedge \cdots \wedge\left(0, Y_{a}\right) \\
& \wedge\left(0, \zeta_{1}\right) \wedge \cdots \wedge\left(0, \zeta_{c}\right) \wedge(\bar{V}, 0) .
\end{aligned}
$$

From $(\mathrm{I}-1), T\left(Y_{\delta}^{\perp}, 0\right)=\left(\left[V, Y_{\delta}^{\perp}\right], 0\right), \delta=1, \ldots, b$. Let

$$
M_{\delta, \beta}=\left\langle\zeta_{\beta},\left[V, Y_{\delta}^{\perp}\right]\right\rangle
$$

for $\beta, \delta=1, \ldots, b$. Then $\left[V, Y_{\delta}^{\perp}\right]=\sum_{\beta=1}^{b} M_{\delta, \beta} \zeta_{\beta}$. Thus $T\left(Y_{1}^{\perp}, 0\right) \wedge \ldots \wedge$ $T\left(Y_{b}^{\perp}, 0\right)=\operatorname{det}(M)\left(\zeta_{1}, 0\right) \wedge \ldots \wedge\left(\zeta_{b}, 0\right)$. We now must compute $T \mathcal{V} \wedge \mathcal{F}=$

$$
\begin{aligned}
& \operatorname{det}(M)\left(\zeta_{1}, 0\right) \wedge \cdots \wedge\left(\zeta_{b}, 0\right) \wedge T\left(0, Y_{1}^{\perp}\right) \wedge \cdots \wedge T\left(0, Y_{b}^{\perp}\right) \\
& \wedge T\left(0, Y_{1}\right) \wedge \cdots \wedge T\left(0, Y_{a}\right) \\
& \wedge T\left(0, \zeta_{1}\right) \wedge \cdots \wedge T\left(0, \zeta_{c}\right) \wedge(0, \bar{V}) \wedge\left(0, Y_{1}\right) \wedge \cdots \wedge\left(0, Y_{a}\right) \\
& \wedge\left(0, \zeta_{1}\right) \cdots \wedge\left(0, \zeta_{c}\right) \wedge(\bar{V}, 0) .
\end{aligned}
$$


By $(\mathrm{I}-2), T\left(0, Y_{\delta}^{\perp}\right)=\left(-\tau Y_{\delta}^{\perp}+\frac{\tau}{2}\left[V, Y_{\delta}^{\perp}\right], 0\right), \delta=1, \ldots, b$, and $T\left(0, Y_{\alpha}\right)=$ $\left(-\tau Y_{\alpha}, 0\right), \alpha=1, \ldots, a$. Since $\left[V, Y_{\delta}^{\perp}\right] \in \operatorname{span}\left\{\zeta_{1}, \ldots, \zeta_{b}\right\}$, and since $T\left(0, Y_{\delta}^{\perp}\right)$ is then wedged with $\left(\zeta_{1}, 0\right) \wedge \cdots \wedge\left(\zeta_{b}, 0\right)$, we have up to $\operatorname{sign} T \mathcal{V} \wedge \mathcal{F}=$

$$
\begin{aligned}
& \operatorname{det}(M)\left(\zeta_{1}, 0\right) \wedge \cdots \wedge\left(\zeta_{b}, 0\right) \wedge \tau^{b}\left(Y_{1}^{\perp}, 0\right) \wedge \cdots \wedge\left(Y_{b}^{\perp}, 0\right) \\
& \wedge \tau^{a}\left(Y_{1}, 0\right) \wedge \cdots \wedge\left(Y_{a}, 0\right) \\
& \wedge T\left(0, \zeta_{1}\right) \wedge \cdots \wedge T\left(0, \zeta_{c}\right) \wedge(0, \bar{V}) \wedge\left(0, Y_{1}\right) \wedge \cdots \wedge\left(0, Y_{1}\right) \\
& \wedge\left(0, \zeta_{1}\right) \wedge \cdots \wedge\left(0, \zeta_{c}\right) \wedge(\bar{V}, 0) .
\end{aligned}
$$

By $(\mathrm{I}-3), T\left(0, \zeta_{\beta}\right)=\left(\frac{-\tau}{2} j\left(\zeta_{\beta}\right) V-\tau \zeta_{\beta}+\frac{\tau}{6}\left[V, j\left(\zeta_{b}\right) V\right],-j\left(\zeta_{b}\right) V\right)$, for $\beta=$ $1, \ldots, b$ and $T\left(0, \zeta_{\beta}\right)=\left(-\tau \zeta_{\beta}, 0\right)$, for $\beta=b+1, \ldots, c$. Since $j\left(\zeta_{b}\right) V \in$ $\operatorname{span}\left\{Y_{1}^{\perp}, \ldots, Y_{b}^{\perp}, Y_{1}, \ldots, Y_{a}\right\}$, and since $T\left(0, \zeta_{\beta}\right)$ is then wedged with $\left(Y_{1}^{\perp}, 0\right)$ $\wedge \cdots \wedge\left(Y_{a}, 0\right)$, we can ignore the contribution of $j\left(\zeta_{\beta}\right) V$ in the left component, $\beta=1, \ldots, b$. Likewise, $\zeta_{\beta}$ and $\left[V, j\left(\zeta_{\beta}\right) V\right]$ in the left component are included in spans of other elements, for $\beta=1, \ldots, b$; the only contribution of $T\left(0, \zeta_{\beta}\right)$ to $T \mathcal{V} \wedge \mathcal{F}$ is $\left(0, j\left(\zeta_{\beta}\right) V\right)$, for $\beta=1, \ldots, b$.

Now $j\left(\zeta_{\beta}\right) V=\sum_{\delta=1}^{b} M_{\delta, \beta} Y_{\delta}^{\perp}$. So we have $T \mathcal{V} \wedge \mathcal{F}=$

$$
\begin{aligned}
& \operatorname{det}(M) \tau^{a+b}\left(\zeta_{1}, 0\right) \wedge \cdots \wedge\left(\zeta_{b}, 0\right) \wedge\left(Y_{1}^{\perp}, 0\right) \wedge \cdots \wedge\left(Y_{b}^{\perp}, 0\right) \\
& \wedge\left(Y_{1}, 0\right) \wedge \cdots \wedge\left(Y_{a}, 0\right) \\
& \wedge \tau^{c-b} \operatorname{det}(M)\left(0, Y_{1}^{\perp}\right) \wedge \cdots \wedge\left(0, Y_{b}^{\perp}\right) \wedge\left(\zeta_{b+1}, 0\right) \wedge \cdots \wedge\left(\zeta_{c}, 0\right) \\
& \wedge(0, \bar{V}) \wedge\left(0, Y_{1}\right) \wedge \cdots \wedge\left(0, Y_{1}\right) \wedge\left(0, \zeta_{1}\right) \wedge \cdots \wedge\left(0, \zeta_{c}\right) \wedge(\bar{V}, 0)
\end{aligned}
$$

In conclusion, the DG-multiplier for $d \mu$ when $\gamma=\exp (V)$ and $\tau=|V|$ is:

$$
\frac{1}{\tau^{(\operatorname{dim} C(\gamma, G)-1) / 2}|\operatorname{det} M|},
$$

where $M$ is defined in (I-M).

Case II: Poincaré Map. $\gamma=\exp (Z)$ and $\tau=|Z|$

Let $\bar{Z}=Z / \tau=Z /|Z|$. By Theorem 2.12, $\tilde{F}(\tau, \gamma)=G \times\{\bar{Z}\}$. Let $v_{\tilde{p}} \in \tilde{F}(\tau, \gamma)$, so $v_{\tilde{p}}=(\tilde{p}, \bar{Z})$ where $\tilde{p} \in G$. Then

$$
T_{v_{\tilde{p}}} \tilde{F}(\tau, \gamma)=\mathfrak{g} \times\{0\} .
$$

So $\tilde{\Phi}_{\tau *_{\tilde{p}}}=\operatorname{Id}$ when restricted to $\mathfrak{g} \times\{0\}$. We must calculate $(1) \tilde{\Phi}_{\tau *_{\tilde{p}}}(0, U)$ where $U \in \mathfrak{v}$ and $(2) \tilde{\Phi}_{\tau *_{\tilde{p}}}(0, \zeta)$ where $\zeta \in \mathfrak{z}$.

For $(1)$, we let $\alpha(s)=(\tilde{p}, \sin s U+\cos s \bar{Z})$, so that $a_{1}(s)=e, a_{2}(s)=$ $\sin s U+\cos s \bar{Z}, \dot{a}_{1}(0)=0$ and $\dot{a}_{2}(0)=U$. Here $Z_{0}^{s}=\cos s \bar{Z}$, so $\vartheta_{m}^{s}=$ 
$\vartheta_{m}\left(Z_{0}^{s}\right)=\cos s \vartheta_{m}(\bar{Z})$, and $\dot{\vartheta}_{m}^{0}=0$ for $m \in \mathcal{M}(Z)$. Also, $\vartheta_{m}^{0}=0$ if and only if $\vartheta_{m}(\bar{Z})=0$, so $N=\emptyset$ and $M=\mathcal{M}$. Let

$$
U=X_{1}+\sum_{m \in \mathcal{M}} \xi_{m}
$$

where $X_{1} \in \operatorname{ker} j(Z)$ and $\xi_{m} \in W_{m}(Z)$. Let $\vartheta_{m}=\vartheta_{m}(\bar{Z})=\vartheta_{m}(Z) / \tau$ and $\Xi_{m}=j(\bar{Z}) \xi_{m} / \vartheta_{m}$. Then $X_{1}^{s}=\sin s X_{1}$ and $X_{2}^{s}=\sum_{m \in \mathcal{M}} \sin s \xi_{m}$, so that $\xi_{m}^{s}=\sin s \xi_{m}$. From this we have $X_{1}^{0}=0, \xi_{m}^{0}=0, \Xi_{m}^{0}=0, \dot{Z}_{0}^{0}=0, \dot{X}_{1}^{0}=$ $X_{1}, \dot{\xi}_{m}^{0}=\xi_{m}$ and $\dot{\Xi}_{m}^{0}=\Xi_{m}$. This implies $M^{\prime}=\emptyset$ and $M^{\prime \prime}=\mathcal{M}$. Plugging these into $(2.21)$ we have $\dot{X}^{0}(\tau)=\tau X_{1}+\sum_{m \in \mathcal{M}} \tau \frac{\left(1-\cos \left(\vartheta_{m}(Z)\right)\right)}{\vartheta_{m}(Z)} \Xi_{m}+$ $\sum_{m \in \mathcal{M}} \tau \frac{\sin \left(\vartheta_{m}(Z)\right)}{\vartheta_{m}(Z)} \xi_{m}, \quad-\dot{X}^{\prime} 0(\tau)+\dot{X}_{0}^{0}=-\sum_{m \in \mathcal{M}} \sin \left(\vartheta_{m}(Z)\right) \Xi_{m}+\sum_{m \in \mathcal{M}}$ $\left(1-\cos \left(\vartheta_{m}(Z)\right)\right) \xi_{m}$ and $\dot{Z}^{0}(\tau)=0$. By $(2.18)$

$$
\begin{aligned}
\left(\mathrm{Id}-\tilde{\Phi}_{\tau *_{\tilde{p}}}\right)(0, U)= & \left(-\tau X_{1}+\sum_{m \in \mathcal{M}} \tau \frac{\left(\cos \left(\vartheta_{m}(Z)\right)-1\right)}{\vartheta_{m}(Z)} \Xi_{m}\right. \\
& -\sum_{m \in \mathcal{M}} \tau \frac{\sin \left(\vartheta_{m}(Z)\right)}{\vartheta_{m}(Z)} \xi_{m}, \\
& \left.-\sum_{m \in \mathcal{M}} \sin \left(\vartheta_{m}(Z)\right) \Xi_{m}+\sum_{m \in \mathcal{M}}\left(1-\cos \left(\vartheta_{m}(Z)\right)\right) \xi_{m}\right),
\end{aligned}
$$

where $X_{1}$ and $\xi_{m}$ are defined in (II- $U$ ).

For $(2)$, we let $\alpha(s)=(\tilde{p}, \sin s \zeta+\cos s \bar{Z})$, so that $a_{1}(s)=e, a_{2}(s)=$ $\sin s \zeta+\cos s \bar{Z}, \dot{a}_{1}(0)=0$ and $\dot{a}_{2}(0)=\zeta$. Here $Z_{0}^{s}=\sin s \zeta+\cos s \bar{Z}$, so $\dot{Z}_{0}^{0}=$ $\zeta$. Also $X_{0}^{s} \equiv 0$, so $X_{1}^{s} \equiv 0$ and $\xi_{m}^{s} \equiv 0$ for $m \in \mathcal{M}$. From this we have $X_{1}^{0}=0, \xi_{m}^{0}=0, \Xi_{m}^{0}=0, \dot{X}_{1}^{0}=0, \dot{\xi}_{m}^{0}=0$ and $\dot{\Xi}_{m}^{0}=0$ for $m \in \mathcal{M}$. One easily computes that $\dot{X}^{0}(\tau)=0,-\dot{X}^{0}(\tau)+\dot{X}_{0}^{0}=0$ and $\dot{Z}^{0}(\tau)=\tau \zeta$. Thus

$$
\left(\operatorname{Id}-\tilde{\Phi}_{\tau *_{\tilde{p}}}\right)(0, \zeta)=(-\tau \zeta, 0) .
$$

Case II: DG-Multiplier. Let $\left\{\xi_{0}^{1}, \ldots, \xi_{0}^{a_{1}}\right\}$ be an orthonormal basis of $\operatorname{ker} j(Z)$. For $m \in \mathcal{M}$, let $\xi_{m}^{1}$ be a unit vector of $W_{m}(Z)$. Let $\Xi_{m}^{1}=$ $j(Z) \xi_{m}^{1} / \vartheta_{m}(Z)$. One easily checks that $\xi_{m}^{1}$ and $\Xi_{m}^{1}$ are orthogonal unit vectors in $W_{m}(Z)$. Let $\xi_{m}^{2}$ be a unit vector in $W_{m}(Z)$ that is orthogonal to both $\xi_{m}^{1}$ and $\Xi_{m}^{1}$. Proceed until we have $\left\{\xi_{m}^{1}, \Xi_{m}^{1}, \xi_{m}^{2}, \Xi_{m}^{2}, \ldots, \xi_{m}^{a_{m}}, \Xi_{m}^{a_{m}}\right\}$, an orthonormal basis of $W_{m}(Z)$ such that $\Xi_{m}^{\alpha}=j(Z) \xi_{m}^{\alpha} / \vartheta_{m}(Z), \alpha=1, \ldots, a_{m}$. Let $\left\{\bar{Z}, \zeta_{1}, \ldots, \zeta_{b}\right\}$ be an orthonormal basis of $\mathfrak{z}$. 
We set

$$
\mathcal{E}=\left\{\left(\xi_{0}^{1}, 0\right), \ldots,\left(\Xi_{\mu}^{a_{\mu}}, 0\right),(\bar{Z}, 0),\left(\zeta_{1}, 0\right), \ldots,\left(\zeta_{b}, 0\right),(0, \bar{Z})\right\}
$$

By Theorem 2.20,

$$
\mathcal{F}=\left\{\left(0, \xi_{0}^{1}\right), \ldots,\left(0, \Xi_{\mu}^{a_{\mu}}\right),(0, \bar{Z}),\left(0, \zeta_{1}\right), \ldots,\left(0, \zeta_{b}\right),(-\bar{Z}, 0)\right\}
$$

We set

$$
\mathcal{V}=\left\{\left(0, \xi_{0}^{1}\right), \ldots,\left(0, \Xi_{\mu}^{a_{\mu}}\right),\left(0, \zeta_{1}\right), \ldots,\left(0, \zeta_{b}\right)\right\}
$$

We must calculate $T \mathcal{V} \wedge \mathcal{F}$ as a multiple of $\mathcal{V} \wedge \mathcal{E}$, up to sign. We have

$$
\begin{aligned}
T \mathcal{V} \wedge \mathcal{F}= & T\left(0, \xi_{0}^{1}\right) \wedge \cdots \wedge T\left(0, \Xi_{\mu}^{a_{\mu}}\right) \wedge T\left(0, \zeta_{1}\right) \wedge \cdots \wedge T\left(0, \zeta_{b}\right) \\
& \wedge\left(0, \xi_{0}^{1}\right) \wedge \cdots \wedge\left(0, \Xi_{\mu}^{a_{\mu}}\right) \wedge(0, \bar{Z}) \wedge\left(0, \zeta_{1}\right) \wedge \cdots \wedge\left(0, \zeta_{b}\right) \wedge(-\bar{Z}, 0)
\end{aligned}
$$

By $\quad(\mathrm{II}-1) \quad T\left(0, \xi_{0}^{1}\right) \wedge \cdots \wedge T\left(0, \xi_{0}^{a_{0}}\right)= \pm \tau^{a_{0}}\left(\xi_{0}^{1}, 0\right) \wedge \cdots \wedge\left(\xi_{0}^{a_{0}}, 0\right), \quad$ and from (II-2), $T\left(0, \zeta_{1}\right) \wedge \cdots \wedge T\left(0, \zeta_{b}\right)= \pm \tau^{b}\left(\zeta_{1}, 0\right) \wedge \cdots \wedge\left(\zeta_{b}, 0\right)$. Now $T\left(0, \xi_{m}^{\alpha}\right)$ $=\left(-c_{m} \tau \Xi_{m}^{\alpha}-s_{m} \tau \xi_{m}^{\alpha}, *\right)$, where $c_{m}=\left(1-\cos \left(\vartheta_{m}(Z)\right)\right) / \vartheta_{m}(Z)$ and $s_{m}=$ $\sin \left(\vartheta_{m}(Z)\right) / \vartheta_{m}(Z)$. The right-hand component of $T\left(0, \xi_{m}^{\alpha}\right)$ is contained in $W_{m}(Z)$, and as in Case I, because the result is ultimately wedged with $\left(0, \xi_{1}^{1}\right) \wedge \cdots \wedge\left(0, \Xi_{\mu}^{a_{\mu}}\right)$, the right-hand component contributes nothing to the final wedge product. Note that $j(Z) \Xi_{m}^{\alpha} / \vartheta_{m}(Z)=-\xi_{m}^{\alpha}$. So, $T\left(0, \Xi_{m}^{\alpha}\right)=$ $\left(-s_{m} \tau \Xi_{m}^{\alpha}+c_{m} \tau \xi_{m}^{\alpha}, *\right)$, and the right-hand component of $T\left(0, \Xi_{m}^{\alpha}\right)$ also lies in $W_{m}(Z)$. Thus the contribution of $T\left(0, \xi_{m}^{\alpha}\right) \wedge T\left(0, \Xi_{m}^{\alpha}\right)$ to $T \mathcal{V} \wedge \mathcal{F}$ is $\tau^{2}\left(s_{m}^{2}+c_{m}^{2}\right)\left(\xi_{m}^{\alpha}, 0\right) \wedge\left(\Xi_{m}^{\alpha}, 0\right)$.

Putting this altogether, we conclude that the DG-multiplier for $d \mu$ when $\gamma=\exp (Z)$ and $\tau=|Z|$ is

$$
\tau^{-(\operatorname{dim} \mathfrak{g}-1) / 2} \prod_{m \in \mathcal{M}(Z)}\left(\frac{2\left(1-\cos \left(\vartheta_{m}(Z)\right)\right.}{\vartheta_{m}(Z)^{2}}\right)^{-\operatorname{dim} W_{m}(Z) / 4}
$$

Note that by the Clean Intersection Hypothesis (Theorem 2.14), $\left(1-\cos \left(\tau \vartheta_{m}(Z)\right)\right) \neq 0$ for all $m \in \mathcal{M}$ and for all $Z \in \log \mathfrak{g} \cap \mathfrak{z}$.

Case III: Poincaré Map. $\gamma=\exp (Z)$ and $\tau<|Z|$.

By Theorem 2.11, the periods of $\gamma$ with $\tau<|Z|$ are precisely

$$
\left\{\frac{|Z|}{\left|K\left(X_{2}+Z_{0}\right)\right|}: X_{2}+Z_{0} \text { satisfy (i)-(iv) below }\right\}
$$


Given $Z_{0} \in \mathfrak{z}$ and $X_{2}=\sum_{m \in \mathcal{M}\left(Z_{0}\right)} \xi_{m} \in \mathfrak{v}$, where $\xi_{m} \in W_{m}\left(Z_{0}\right)$, the conditions referred to above are the following:

(i) $\left|X_{2}+Z_{0}\right|=1$,

(ii) $X_{2} \perp \operatorname{ker} j\left(Z_{0}\right), X_{2} \neq 0$,

(iii) $Z \in \operatorname{span}_{\mathbb{R}^{+}}\left\{K\left(X_{2}+Z_{0}\right)\right\}$,

(iv) for all $m \in \mathcal{M}$ such that $\xi_{m} \neq 0, \frac{|Z| \vartheta_{m}\left(Z_{0}\right)}{2 \pi\left|K\left(X_{2}+Z_{0}\right)\right|} \in \mathbb{Z}$.

Let $\tau=\frac{|Z|}{K\left(\hat{X}_{2}+\hat{Z}_{0}\right)}$ where $\hat{X}_{2}+\hat{Z}_{0}$ satisfies (i)-(iv) above. Then by Theorem 2.12

$$
\tilde{F}(\tau, \gamma)=\left\{v_{\tilde{p}}: v=X_{2}+Z_{0}: X_{2}+Z_{0} \text { satisfy (a)-(d) below }\right\} .
$$

Given $\tilde{p} \in G, Z_{0} \in \mathfrak{z}$ and $X_{2}=\sum_{m \in \mathcal{M}\left(Z_{0}\right)} \xi_{m} \in \mathfrak{v}$, where $\xi_{m} \in W_{m}\left(Z_{0}\right)$, the conditions referred to above are the following:

(a) $\left|X_{2}+Z_{0}\right|=1$,

(b) $X_{2} \perp \operatorname{ker} j\left(Z_{0}\right), X_{2} \neq 0$,

(c) $K\left(X_{2}+Z_{0}\right)=\frac{1}{\tau} Z$ and

(d) for all $m \in \mathcal{M}\left(Z_{0}\right)$ such that $\xi_{m} \neq 0, \vartheta_{m}\left(Z_{0}\right) \in \frac{2 \pi}{\tau} \mathbb{Z}^{+}$.

Let $v_{\tilde{p}} \in \tilde{F}(\tau, \gamma)$, so $v_{\tilde{p}}=\left(\tilde{p}, X_{2}+Z_{0}\right)$ where $\tilde{p} \in G$ and $v=X_{2}+Z_{0}$ satisfies $(a)-(d)$ above. Note that since there is no restriction on $\tilde{p}$ in $\tilde{F}(\tau, \gamma)$, $\mathfrak{g} \times\{0\} \subset T_{v_{\tilde{p}}} \tilde{F}(\tau, \gamma)$, so $\tilde{\Phi}_{\tau *_{\tilde{p}}}=\mathrm{Id}$ when restricted to $\mathfrak{g} \times\{0\}$. Elements not in $T_{v_{\tilde{p}}} \tilde{F}(\tau, \gamma)$ are spanned by vectors of the following type: (1) $\tilde{\Phi}_{\tau *_{\tilde{p}}}$ $\left(0, v^{\perp}\right)$ where $v^{\perp} \in \operatorname{span}_{\mathbb{R}}\left\{X_{2}, Z_{0}\right\}, v^{\perp} \perp v,(2) \tilde{\Phi}_{\tau *_{\tilde{p}}}(0, U)$ where $U \in \mathfrak{v}, U \perp$ $v,(3) \tilde{\Phi}_{\tau *_{\tilde{p}}}(0, \zeta)$ where $\zeta \in \mathfrak{z}, \zeta \perp v$.

For (1), let $\bar{X}_{2}=X_{2} /\left|X_{2}\right|$ and $\bar{Z}_{0}=Z_{0} /\left|Z_{0}\right|$. As $\left|X_{2}+Z_{0}\right|=1$, there exists $\beta$ so that $v=\cos \beta \bar{X}_{2}+\sin \beta \bar{Z}_{0}$. In particular, $\cos \beta=\left|X_{2}\right|$ and $\sin \beta=\left|Z_{0}\right|$. Recall that $X_{2}=\sum_{m \in \mathcal{M}} \xi_{m}$ where $\xi_{m} \in W_{m}\left(Z_{0}\right)$. Let $\bar{X}_{2}=$ $\sum_{m \in \mathcal{M}} \bar{\xi}_{m}$, where $\bar{\xi}_{m} \in W_{m}\left(Z_{0}\right)$, so that $\bar{\xi}_{m}=\xi_{m} /\left|X_{2}\right|$. For $m \in \mathcal{M}\left(Z_{0}\right)$, let $\bar{\vartheta}_{m}=\vartheta_{m}\left(\bar{Z}_{0}\right), \bar{J}=j\left(\bar{Z}_{0}\right)$ and $\bar{\Xi}_{m}=\bar{J} \xi_{m}^{-} / \bar{\vartheta}_{m}$. Set $v^{\perp}=-\sin \beta \bar{X}_{2}+\cos \beta \bar{Z}_{0}$. Now let $\alpha(s)=\left(\tilde{p}, \cos (\beta+s) \bar{X}_{2}+\sin (\beta+s) \bar{Z}_{0}\right)$, so that $a_{1}(s)=e, a_{2}(s)=$ $\cos (\beta+s) \bar{X}_{2}+\sin (\beta+s) \bar{Z}_{0}, \quad \dot{a}_{1}(0)=0$ and $\dot{a}_{2}(0)=v^{\perp}$. Here $Z_{0}^{s}=$ $\sin (\beta+s) \bar{Z}_{0}$, so $\vartheta_{m}^{s}=\vartheta_{m}\left(Z_{0}^{s}\right)=\sin (\beta+s) \bar{\vartheta}_{m}$ and $\dot{\vartheta}_{m}^{0}=\cos \beta \bar{\vartheta}_{m}$ for $m \in$ $\mathcal{M}$. Also, as $\sin \beta \neq 0, \vartheta_{m}^{0}=0$ if and only if $\bar{\vartheta}_{m}=0$, so $N=\emptyset$ and $M=\mathcal{M}$. Then $X_{1}^{s}=0, X_{2}^{s}=\cos (\beta+s) \bar{X}_{2}$ so that $\xi_{m}^{s}=\cos (\beta+s) \bar{\xi}_{m}$ and $\Xi_{m}^{s}=$ $\cos (\beta+s) \bar{\Xi}_{m}$. From this we have $X_{1}^{0}=0, \xi_{m}^{0}=\cos \beta \bar{\xi}_{m}, \Xi_{m}^{0}=\cos \beta \bar{\Xi}_{m}$, 
$\dot{Z}_{0}^{0}=\cos \beta \bar{Z}_{0}$ and $\dot{X}_{1}^{0}=0$. Also, $\dot{\xi}_{m}^{0}=-\sin \beta \bar{\xi}_{m}$ and $\dot{\Xi}_{m}^{0}=-\sin \beta \bar{\Xi}_{m}$. This implies that if $\xi_{m}^{0}=0$ then $\dot{\xi}_{m}^{0}=0$, so that the terms with $m \in M^{\prime \prime}$ contribute nothing to the following calculations. Plugging these into $(2.21)$ we have $\dot{X}^{0}(\tau)=\tau \cos \beta \cot \beta \bar{X}_{2},-\dot{X}^{\prime 0}(\tau)+\dot{X}_{0}^{0}=-\tau \cos ^{2} \beta \bar{J} \bar{X}_{2}$ and $\dot{Z}^{0}(\tau)=$ $\tau \cos \beta \bar{Z}_{0}+\tau \cos \beta \csc ^{2} \beta \sum_{m \in \mathcal{M}}\left[\bar{\xi}_{m}, \bar{J}^{-1} \bar{\xi}_{m}\right]$. By (2.18),

$$
\begin{aligned}
\left(\operatorname{Id}-\tilde{\Phi}_{\tau *_{\tilde{p}}}\right)\left(0, v^{\perp}\right)= & \left(\operatorname{Id}-\tilde{\Phi}_{\tau *_{\tilde{p}}}\right)\left(0,-\frac{\left|Z_{0}\right|}{\left|X_{2}\right|} X_{2}+\frac{\left|X_{2}\right|}{\left|Z_{0}\right|} Z_{0}\right) \\
= & \left(-\tau \frac{\left|X_{2}\right|}{\left|Z_{0}\right|} X_{2}-\tau \frac{\left|X_{2}\right|}{\left|Z_{0}\right|} Z_{0}-\frac{\tau}{\left|X_{2}\right|\left|Z_{0}\right|} \sum_{m \in \mathcal{M}}\left[\xi_{m}, J^{-1} \xi_{m}\right],\right. \\
(\text { III-1) } & \left.-\tau \frac{\left|X_{2}\right|}{\left|Z_{0}\right|} J X_{2}\right) .
\end{aligned}
$$

For $(2)$, we let $\alpha(s)=\left(\tilde{p}, \sin s U+\cos s\left(X_{2}+Z_{0}\right)\right)$, so that $a_{1}(s)=e$, $a_{2}(s)=\sin s U+\cos s\left(X_{2}+Z_{0}\right), \quad \dot{a}_{1}(0)=0$ and $\dot{a}_{2}(0)=U$. Recall that $X_{2}=\sum_{m \in \mathcal{M}} \xi_{m}$, where $\xi_{m} \in W_{m}\left(Z_{0}\right)$ and let $\vartheta_{m}=\vartheta_{m}\left(Z_{0}\right)$, let $\Xi_{m}=$ $j\left(Z_{0}\right) \xi_{m} / \vartheta_{m}$ for $m \in \mathcal{M}$. Let

$$
U=X_{1}^{u}+\sum_{m \in M} \xi_{m}^{u} \quad \text { and } \quad \Xi_{m}^{u}=\frac{j\left(Z_{0}\right) \xi_{m}^{u}}{\vartheta_{m}\left(Z_{0}\right)}
$$

where $X_{1}^{u} \in \operatorname{ker} j\left(Z_{0}\right)$ and $\xi_{m}^{u} \in W_{m}\left(Z_{0}\right)$ for $m \in \mathcal{M}$. Here $Z_{0}^{s}=\cos s Z_{0}$, so $\vartheta_{m}^{s}=\vartheta_{m}\left(Z_{0}^{s}\right)=\cos s \vartheta_{m}$, and $\dot{\vartheta}_{m}^{0}=0$ for $m \in \mathcal{M}$. Also, $\vartheta_{m}^{0}=0$ if and only if $\vartheta_{m}=0$, so $N=\emptyset$ and $M=\mathcal{M}$. Now $X_{1}^{s}=\sin s X_{1}^{u}$ and $X_{2}^{s}=\sum_{m \in \mathcal{M}}\left(\cos s \xi_{m}+\sin s \xi_{m}^{u}\right)$ so that $\xi_{m}^{s}=\cos s \xi_{m}+\sin s \xi_{m}^{u}$ and $\Xi_{m}^{s}=$ $j\left(Z_{0}^{s}\right) \xi_{m}^{s} / \vartheta_{m}^{s}=\cos s \Xi_{m}+\sin s \Xi_{m}^{u}$. From this we have $X_{1}^{0}=0, X_{2}^{0}=X_{0}$ and $\xi_{m}^{0}=\xi_{m}, \quad \Xi_{m}^{0}=\Xi_{m}, \quad \dot{Z}_{0}^{0}=0, \quad \dot{X}_{1}^{0}=X_{1}^{u}, \quad \dot{\xi}_{m}^{0}=\xi_{m}^{u}$ and $\dot{\Xi}_{m}^{0}=\Xi_{m}^{u}$. Note that $\xi_{m}$ and $\xi_{m}^{u}$ are not, in general, related so that $M^{\prime \prime}$ is a consideration in what follows. Plugging these into (2.21) we have $\dot{X}^{0}(\tau)=\tau X_{1}^{u}+\sum_{m \in \mathcal{M}} \frac{\left(1-\cos \left(\tau \vartheta_{m}\right)\right)}{\vartheta_{m}} \Xi_{m}^{u}+\sum_{m \in \mathcal{M}} \frac{\sin \left(\tau \vartheta_{m}\right)}{\vartheta_{m}} \xi_{m}^{u}, \quad-\dot{X}^{\prime} 0(\tau)+$ $\dot{X}_{0}^{0}=-\sum_{m \in \mathcal{M}} \sin \left(\tau \vartheta_{m}\right) \Xi_{m}^{u}+\sum_{m \in \mathcal{M}}\left(1-\cos \left(\tau \vartheta_{m}\right)\right) \xi_{m}^{u}$, and

$$
\begin{aligned}
\dot{Z}^{0}(\tau)= & -\sum_{m \in \mathcal{M}} \frac{\tau}{\vartheta_{m}}\left[X_{1}^{u}, \Xi_{m}\right]+\sum_{m \in \mathcal{M}} \frac{\tau}{2 \vartheta_{m}}\left(\left[\xi_{m}^{u}, \Xi_{m}\right]+\left[\xi_{m}, \Xi_{m}^{u}\right]\right) \\
& +\sum_{m, h \in \mathcal{M}, m \neq h} \frac{\vartheta_{h}\left(1-\cos \left(\tau \vartheta_{m}\right)\right)}{\vartheta_{m}\left(\vartheta_{m}^{2}-\vartheta_{h}^{2}\right)}\left[\Xi_{m}^{u}, \Xi_{h}\right] \\
& -\sum_{m, h \in \mathcal{M}, m \neq h} \frac{\sin \left(\tau \vartheta_{m}\right)}{\vartheta_{m}^{2}-\vartheta_{h}^{2}}\left[\Xi_{m}^{u}, \xi_{h}\right]
\end{aligned}
$$




$$
\begin{aligned}
& +\sum_{m, h \in \mathcal{M}, m \neq h} \frac{\vartheta_{h} \sin \left(\tau \vartheta_{m}\right)}{\vartheta_{m}\left(\vartheta_{m}^{2}-\vartheta_{h}^{2}\right)}\left[\xi_{m}^{u}, \Xi_{h}\right] \\
& +\sum_{m, h \in \mathcal{M}, m \neq h} \frac{\left(1-\cos \left(\tau \vartheta_{m}\right)\right)}{\vartheta_{m}^{2}-\vartheta_{h}^{2}}\left[\xi_{m}^{u}, \xi_{h}\right] .
\end{aligned}
$$

Thus

$$
\begin{aligned}
\left(\operatorname{Id}-\tilde{\Phi}_{\tau *_{\tilde{p}}}\right)(0, U)= & \left(-\tau X_{1}^{u}+\sum_{m \in \mathcal{M}} \frac{\left(\cos \left(\tau \vartheta_{m}\right)-1\right)}{\vartheta_{m}} \Xi_{m}^{u}-\sum_{m \in \mathcal{M}} \frac{\sin \left(\tau \vartheta_{m}\right)}{\vartheta_{m}} \xi_{m}^{u}\right. \\
& -\tau\left[X_{1}^{u}, J^{-1} X_{2}\right]-\sum_{m \in \mathcal{M}} \frac{\tau}{2 \vartheta_{m}}\left(\left[\xi_{m}^{u}, \Xi_{m}\right]+\left[\xi_{m}, \Xi_{m}^{u}\right]\right) \\
& +\sum_{m, h \in \mathcal{M}, m \neq h} \frac{\vartheta_{h}\left(\cos \left(\tau \vartheta_{m}\right)-1\right)}{\vartheta_{m}\left(\vartheta_{m}^{2}-\vartheta_{h}^{2}\right)}\left[\Xi_{m}^{u}, \Xi_{h}\right] \\
& +\sum_{m, h \in \mathcal{M}, m \neq h} \frac{\sin \left(\tau \vartheta_{m}\right)}{\vartheta_{m}^{2}-\vartheta_{h}^{2}}\left[\Xi_{m}^{u}, \xi_{h}\right] \\
& -\sum_{m, h \in \mathcal{M}, m \neq h} \frac{\vartheta_{h} \sin \left(\tau \vartheta_{m}\right)}{\vartheta_{m}\left(\vartheta_{m}^{2}-\vartheta_{h}^{2}\right)}\left[\xi_{m}^{u}, \Xi_{h}\right] \\
& +\sum_{m, h \in \mathcal{M}, m \neq h} \frac{\left(\cos \left(\tau \vartheta_{m}\right)-1\right)}{\vartheta_{m}^{2}-\vartheta_{h}^{2}}\left[\xi_{m}^{u}, \xi_{h}\right], \\
& \left.-\sum_{m \in \mathcal{M}} \sin \left(\tau \vartheta_{m}\right) \Xi_{m}^{u}+\sum_{m \in \mathcal{M}}\left(1-\cos \left(\tau \vartheta_{m}\right)\right) \xi_{m}^{u}\right),
\end{aligned}
$$

where $\vartheta_{m}=\vartheta_{m}\left(Z_{0}\right)$ and $\xi_{m}^{u}$ and $\Xi_{m}^{u}$ are given in (III- $U$ ).

For $(3)$, we let $\alpha(s)=\left(\tilde{p}, \cos s\left(X_{2}+Z_{0}\right)+\sin s \zeta\right)$, so that $a_{1}(s)=e$, $a_{2}(s)=\cos s\left(X_{2}+Z_{0}\right)+\sin s \zeta, \dot{a}_{1}(0)=0$ and $\dot{a}_{2}(0)=\zeta$. Recall that $X_{2}=$ $\sum_{m \in \mathcal{M}} \xi_{m}$, where $\xi_{m} \in W_{m}\left(Z_{0}\right)$, and let $\vartheta_{m}=\vartheta_{m}\left(Z_{0}\right)$ and $\Xi_{m}=$ $j\left(Z_{0}\right) \xi_{m} / \vartheta_{m}$. Here $Z_{0}^{s}=\cos s Z_{0}+\sin s \zeta$, so $\vartheta_{m}^{s}=\vartheta_{m}\left(Z_{0}^{s}\right)$ and $\vartheta_{m}^{0}=\vartheta_{m}$ for $m \in \mathcal{M}$, but $\dot{\vartheta}_{m}^{0}$ cannot, in general, be simplified. Let $X_{1}^{s}$ denote the component of $\cos s X_{2}$ in $\operatorname{ker} j\left(Z_{0}^{s}\right)$. Note that $X_{1}^{0}=0$ and $\dot{X}_{1}^{0} \in \operatorname{ker} j\left(Z_{0}\right)$. Let $\xi_{m}^{s}$ denote the component of $\cos s X_{2}$ in $W_{m}\left(Z_{0}^{s}\right)$. Let $\Xi_{m}^{s}$ denote $j\left(Z_{0}^{s}\right) \xi_{m}^{s} / \vartheta_{m}^{s}$. Note that if $Z_{0} \notin \mathcal{U}$ (see Definition 2.18), then $\vartheta_{m}^{s}-\vartheta_{h}^{s} \rightarrow 0$ need not imply that $m=h$, and $\vartheta_{m}^{s} \rightarrow 0$ need not imply $\vartheta_{m}=0$. Finally, $\xi_{m}^{0}=0$ need not imply $\dot{\xi}_{m}^{0}=0$. Thus, there is very little simplification that can be 
accomplished from (2.21). We write

(III-3)

$$
\begin{aligned}
& \left(\mathrm{Id}-\tilde{\Phi}_{\tau *_{\tilde{p}}}\right)(0, \zeta) \\
& =\left(-\tau\left(\dot{X}_{1}^{0}+\sum_{n \in N} \dot{\xi}_{n}^{0}\right)-\sum_{m \in M^{\prime}}\left(\frac{\tau \dot{\vartheta}_{m}^{0}}{\vartheta_{m}^{0}}\right) \xi_{m}^{0}\right. \\
& -\left.\sum_{n \in N} \frac{\tau^{2}}{2} \frac{d}{d s}\right|_{0}\left(J_{s} \xi_{n}^{s}\right)+\sum_{m \in M^{\prime \prime}} \frac{\cos \left(\tau \vartheta_{m}^{0}\right)-1}{\vartheta_{m}^{0}} \dot{\Xi}_{m}^{0}-\sum_{m \in M^{\prime \prime}} \frac{\sin \left(\tau \vartheta_{m}^{0}\right)}{\vartheta_{m}^{0}} \dot{\xi}_{m}^{0} \\
& -\tau \zeta+\sum_{m \in M^{\prime}} \frac{\tau}{\vartheta_{m}^{0}}\left[\dot{X}_{1}^{0}+\sum_{n \in N} \dot{\xi}_{n}^{0}, \Xi_{m}^{0}\right]-\sum_{\substack{m \in M, h \in M^{\prime} \\
\vartheta_{m}^{0}=\vartheta_{h}^{0}}} \frac{\tau}{2 \vartheta_{m}^{0}}\left(\left[\dot{\xi}_{m}^{0}, \Xi_{h}^{0}\right]+\left[\xi_{h}^{0}, \dot{\Xi}_{m}^{0}\right]\right) \\
& -\sum_{m \in M^{\prime}, n \in N} \frac{\tau^{2}}{2 \vartheta_{m}^{0}}\left[\Xi_{m}^{0},\left.\frac{d}{d s}\right|_{0}\left(J_{s} \xi_{n}^{s}\right)\right]+\sum_{m \in M^{\prime}, n \in N} \frac{\tau}{\vartheta_{m}^{0}{ }^{2}}\left[\xi_{m}^{0},\left.\frac{d}{d s}\right|_{0}\left(J_{s} \xi_{n}^{s}\right)\right] \\
& -\sum_{\substack{m, h \in M^{\prime} \\
\vartheta_{m}^{0} \neq \vartheta_{h}^{0}}} \frac{\tau\left(\dot{\vartheta}_{h}^{0} \vartheta_{m}^{0}-\vartheta_{h}^{0} \dot{\vartheta}_{m}^{0}\right)}{\vartheta_{h}^{0}\left(\vartheta_{m}^{0}{ }^{2}-\vartheta_{h}^{0}\right)}\left[\Xi_{m}^{0}, \xi_{h}^{0}\right] \\
& -\sum_{\substack{m, h \in M^{\prime} \\
\vartheta_{m}^{0}=\vartheta_{h}^{0}}} \frac{\tau\left(3 \dot{\vartheta}_{h}^{0}+\dot{\vartheta}_{m}^{0}\right)}{4 \vartheta_{m}^{0}{ }^{2}}\left[\Xi_{m}^{0}, \xi_{h}^{0}\right]-\sum_{\substack{m, h \in M^{\prime} \\
\vartheta_{m}^{0}=\vartheta_{h}^{0}}} \frac{\tau^{2}\left(\dot{\vartheta}_{m}^{0}-\dot{\vartheta}_{h}^{0}\right)}{8 \vartheta_{m}^{0}}\left(\left[\Xi_{m}^{0}, \Xi_{h}^{0}\right]+\left[\xi_{m}^{0}, \xi_{h}^{0}\right]\right) \\
& +\sum_{\substack{m \in M^{\prime \prime}, h \in M^{\prime} \\
\vartheta_{m}^{0} \neq \vartheta_{h}^{0}}} \frac{\vartheta_{h}^{0}\left(\cos \left(\tau \vartheta_{m}^{0}\right)-1\right)}{\vartheta_{m}^{0}\left(\vartheta_{m}^{0}{ }^{2}-\vartheta_{h}^{0^{2}}\right)}\left[\dot{\Xi}_{m}^{0}, \Xi_{h}^{0}\right]+\sum_{\substack{m \in M^{\prime \prime}, h \in M^{\prime} \\
\vartheta_{m}^{0} \neq \vartheta_{h}^{0}}} \frac{\sin \left(\tau \vartheta_{m}^{0}\right)}{\vartheta_{m}^{0}{ }^{2}-\vartheta_{h}^{0^{2}}}\left[\dot{\Xi}_{m}^{0}, \xi_{h}^{0}\right] \\
& -\sum_{\substack{m \in M^{\prime \prime}, h \in M^{\prime} \\
\vartheta_{m}^{0} \neq \vartheta_{h}^{0}}} \frac{\vartheta_{h}^{0} \sin \left(\tau \vartheta_{m}^{0}\right)}{\vartheta_{m}^{0}\left(\vartheta_{m}^{0}{ }^{2}-\vartheta_{h}^{0^{2}}\right)}\left[\dot{\xi}_{m}^{0}, \Xi_{h}^{0}\right]+\sum_{\substack{m \in M^{\prime \prime}, h \in M^{\prime} \\
\vartheta_{m}^{0} \neq \vartheta_{h}^{0}}} \frac{\cos \left(\tau \vartheta_{m}^{0}\right)-1}{\vartheta_{m}^{0}-\vartheta_{h}^{0^{2}}}\left[\dot{\xi}_{m}^{0}, \xi_{h}^{0}\right], \\
& -\sum_{m \in M^{\prime}} \tau \dot{\vartheta}_{m}^{0} \Xi_{m}^{0}-\left.\sum_{n \in N} \tau \frac{d}{d s}\right|_{0}\left(J_{s} \xi_{n}^{s}\right)-\sum_{m \in M^{\prime \prime}} \sin \left(\tau \vartheta_{m}^{0}\right) \dot{\Xi}_{m}^{0} \\
& \left.+\sum_{m \in M^{\prime \prime}}\left(1-\cos \left(\tau \vartheta_{m}^{0}\right)\right) \dot{\xi}_{m}^{0}\right)
\end{aligned}
$$

Case III: DG-Multiplier. Given (III-3), we cannot hope to compute a closed form that will apply to all two-step nilmanifolds for the DG-multiplier in this case. However, it is easier than it might appear to apply these computations 
to the case of a one-dimensional center/Heisenberg manifold (see Section 4.1) and a particular example (see Section 4.2).

Case IV: The Poincaré Map. $\gamma=\exp \left(V+Z_{V}+Z_{V}^{\perp}\right), V \neq 0, Z_{V}^{\perp} \neq 0$ and $\tau=\left|V+Z_{V}^{\perp}\right|$.

Let $\bar{V}=V /|V|$ and $\bar{Z}_{V}^{\perp}=Z_{V}^{\perp} /\left|Z_{V}^{\perp}\right|$ and $\overline{V+Z_{V}^{\perp}}=\left(V+Z_{V}^{\perp}\right) /\left|V+Z_{V}^{\perp}\right|$. By Theorem 2.12, $\tilde{F}(\tau, \gamma)=C(\gamma, G) \tilde{q} \times\left\{\overline{V+Z_{V}^{\perp}}\right\}$ where $\tilde{q} \exp \left(V+Z_{V}^{\perp}\right) \tilde{q}^{-1}$ $=\gamma$. Let $v_{\tilde{p}} \in \tilde{F}(\tau, \gamma)$, so $v_{\tilde{p}}=\left(\tilde{p}, \overline{V+Z_{V}^{\perp}}\right)$ and $\tilde{p}=x \tilde{q}$ where $x \in C(\gamma, G)$. So that $\tilde{p} \exp \left(V+Z_{V}^{\perp}\right)=\gamma \tilde{p}$. Then

$$
T_{v_{\tilde{p}}} \tilde{F}(\tau, \gamma)=C(\log \gamma, \mathfrak{g}) \times\{0\} .
$$

As $\tilde{\Phi}_{\tau *_{\tilde{p}}}=\mathrm{Id}$ when restricted to $C(\log \gamma, \mathfrak{g}) \times\{0\}$, we must calculate (1) $\tilde{\Phi}_{\tau *_{\tilde{p}}}(U, 0) \quad$ where $U \perp C(\log \gamma, \mathfrak{g}), \quad(2) \tilde{\Phi}_{\tau *_{\tilde{p}}}\left(0, v^{\perp}\right)$ where $v^{\perp} \in$ $\operatorname{span}_{\mathbb{R}}\left\{V, Z_{V}^{\perp}\right\}, v^{\perp} \perp v,(3) \tilde{\Phi}_{\tau *_{\tilde{p}}}(0, U)$ where $U \in \mathfrak{v}, U \perp v$, and $(4) \tilde{\Phi}_{\tau *_{\tilde{p}}}(0, \zeta)$ where $\zeta \in \mathfrak{z}, \zeta \perp v$.

For $(1)$, we let $\alpha(s)=\left(\tilde{p} \exp (s U), \overline{V+Z_{V}^{\perp}}\right)$, so that $a_{1}(s)=s U$ and $a_{2}(s)=\overline{V+Z_{V}^{\perp}}, \dot{a}_{1}(0)=U$ and $\dot{a}_{2}(0)=0$. Thus $Z_{0}^{s}=Z_{V}^{\perp} / \tau, X_{2}^{s}=0$ and $X_{1}^{s}=V / \tau$. So $\dot{Z}_{0}^{0}=0$ and $\dot{X}_{1}^{0}=0$. Using $(2.21)$, we obtain $\dot{X}^{0}(\tau)=0$, $-\dot{X}^{\prime} 0(\tau)+\dot{X}_{0}^{0}=0$ and $\dot{Z}^{0}(\tau)=0$. Using (2.18), we have

$$
\left(\operatorname{Id}-\tilde{\Phi}_{\tau *_{\tilde{p}}}\right)(U, 0)=([V, U], 0)
$$

Note that $[V, U] \neq 0$ since $U \perp C(\log \gamma, \mathfrak{g})$.

For (2), as $\left|\overline{V+Z_{V}^{\perp}}\right|=1$, there exists $\beta$ so that $\overline{V+Z_{V}^{\perp}}=\cos \beta \bar{V}+$ $\sin \beta \bar{Z}_{V}^{\perp}$. Set $v^{\perp}=-\sin \beta \bar{V}+\cos \beta \bar{Z}_{V}^{\perp}$. Now let $\alpha(s)=(\tilde{p}, \cos (\beta+s) \bar{V}+$ $\left.\sin (\beta+s) \bar{Z}_{V}^{\perp}\right), \quad$ so that $a_{1}(s)=e, \quad a_{2}(s)=\cos (\beta+s) \bar{V}+\sin (\beta+s) \bar{Z}_{V}^{\perp}$, $\dot{a}_{1}(0)=0$ and $\dot{a}_{2}(0)=v^{\perp}$. Here $Z_{0}^{s}=\sin (\beta+s) \bar{Z}_{V}^{\perp}, X_{1}^{s}=\cos (\beta+s) \bar{V}$ and $X_{2}^{s}=0$. From this we have $X_{1}^{0}=\cos \beta \bar{V}, \dot{X}_{1}^{0}=-\sin \beta \bar{V}, Z_{0}^{0}=\sin \beta \bar{Z}_{V}^{\perp}$ and $\dot{Z}_{0}^{0}=\cos \beta \bar{Z}_{V}^{\perp}$. Plugging these into $(2.21)$ we have $\dot{X}^{0}(\tau)=-\tau \sin \beta \bar{V}$, $-\dot{X}^{\prime} 0(\tau)+\dot{X}_{0}^{0}=0$ and $\dot{Z}^{0}(\tau)=\tau \cos \beta \bar{Z}_{V}^{\perp}$. Thus

$$
\left(\operatorname{Id}-\tilde{\Phi}_{\tau *_{\tilde{p}}}\right)\left(0, v^{\perp}\right)=\left(-\tau v^{\perp}, 0\right) .
$$

For $(3)$, we let $\alpha(s)=\left(\tilde{p}, \cos s\left(\overline{V+Z_{V}^{\perp}}\right)+\sin s U\right)$ so that $a_{1}(s)=\tilde{p}, a_{2}(s)$ $=\cos s\left(\overline{V+Z_{V}^{\perp}}\right)+\sin s U, \dot{a}_{1}(0)=0$ and $\dot{a}_{2}(0)=U$. Here $Z_{0}^{s}=\cos s Z_{V}^{\perp} / \tau$, so $\vartheta_{m}^{s}=\cos s \vartheta_{m}\left(Z_{V}^{\perp}\right) / \tau$, and $\dot{\vartheta}_{m}^{0}=0$ for $m \in \mathcal{M}\left(Z_{V}^{\perp}\right)$. Also, $\vartheta_{m}^{0}=0$ if and only if $\vartheta_{m}\left(Z_{V}^{\perp}\right)=0$, so $N=\emptyset$ and $M=\mathcal{M}$. Let

$$
U=X_{1}^{u}+\sum_{m \in \mathcal{M}} \xi_{m}^{u} \quad \text { and } \quad \Xi_{m}^{u}=j\left(Z_{V}^{\perp}\right) \xi_{m}^{u} / \vartheta_{m}\left(Z_{V}^{\perp}\right)
$$


where $X_{1}^{u} \in \operatorname{ker} j\left(Z_{V}^{\perp}\right)$ and $\xi_{m}^{u} \in W_{m}\left(Z_{V}^{\perp}\right)$. Denote $\vartheta_{m}=\vartheta_{m}\left(Z_{V}^{\perp}\right) / \tau$. Then $X_{1}^{s}=\sin s X_{1}^{u}+\cos s V / \tau$ and $X_{2}^{s}=\sum_{m \in \mathcal{M}} \sin s \xi_{m}^{u}$, so that $\xi_{m}^{s}=\sin s \xi_{m}^{u}$ and $\Xi_{m}^{s}=\sin s \Xi_{m}^{u}$ for $m \in \mathcal{M}$. From this we have $X_{1}^{0}=V / \tau, \xi_{m}^{0}=0, \Xi_{m}^{0}=$ $0, \dot{Z}_{0}^{0}=0, \dot{X}_{1}^{0}=X_{1}^{u}, \dot{\xi}_{m}^{0}=\xi_{m}^{u}$ and $\dot{\Xi}_{m}^{0}=\Xi_{m}^{u}$, for $m \in \mathcal{M}$. This implies $M^{\prime}=\emptyset$ and $M^{\prime \prime}=\mathcal{M}$. Plugging these into (2.21) we have $\dot{X}^{0}(\tau)=\tau X_{1}^{u}+$ $\sum_{m \in \mathcal{M}} \frac{\left(1-\cos \left(\tau \vartheta_{m}\right)\right)}{\vartheta_{m}} \Xi_{m}^{u}+\sum_{m \in \mathcal{M}} \frac{\sin \left(\tau \vartheta_{m}\right)}{\vartheta_{m}} \xi_{m}^{u}, \quad-\dot{X}^{\prime 0}(\tau)+\dot{X}_{0}^{0}=-\sum_{m \in \mathcal{M}}$ $\sin \left(\tau \vartheta_{m}\right) \Xi_{m}^{u}+\sum_{m \in \mathcal{M}}\left(1-\cos \left(\tau \vartheta_{m}\right)\right) \xi_{m}^{u}$ and $\dot{Z}^{0}(\tau)=[V, A]$ where

$$
\begin{aligned}
A= & \sum_{m \in M} \frac{2 \sin \left(\tau \vartheta_{m}\right)-\tau \vartheta_{m}\left(\cos \left(\tau \vartheta_{m}\right)+1\right)}{2 \tau \vartheta_{m}^{2}} \Xi_{m}^{u} \\
& +\sum_{m \in M} \frac{2\left(\cos \left(\tau \vartheta_{m}\right)-1\right)+\tau \vartheta_{m} \sin \left(\tau \vartheta_{m}\right)}{2 \tau \vartheta_{m}^{2}} \xi_{m}^{u}
\end{aligned}
$$

Thus

$$
\begin{aligned}
\left(\operatorname{Id}-\tilde{\Phi}_{\tau *_{\tilde{p}}}\right)(0, U)= & \left(-\tau X_{1}^{u}+\sum_{m \in M} \tau \frac{\left(\cos \left(\vartheta_{m}\left(Z_{V}^{\perp}\right)\right)-1\right)}{\vartheta_{m}\left(Z_{V}^{\perp}\right)} \Xi_{m}^{u}\right. \\
& +\sum_{m \in M} \tau \frac{-\sin \left(\vartheta_{m}\left(Z_{V}^{\perp}\right)\right)}{\vartheta_{m}\left(Z_{V}^{\perp}\right)} \xi_{m}^{u} \\
+ & {\left[V, \frac{\tau}{2} X_{1}^{u}+\sum_{m \in M} \tau \frac{-\sin \left(\vartheta_{m}\left(Z_{V}^{\perp}\right)\right)+\vartheta_{m}\left(Z_{V}^{\perp}\right)}{\vartheta_{m}\left(Z_{V}^{\perp}\right)^{2}} \Xi_{m}^{u}\right.} \\
& \left.+\sum_{m \in M} \tau \frac{\left(1-\cos \left(\vartheta_{m}\left(Z_{V}^{\perp}\right)\right)\right)}{\vartheta_{m}\left(Z_{V}^{\perp}\right)^{2}} \xi_{m}^{u}\right] \\
& \left.-\sum_{m \in M} \sin \left(\vartheta_{m}\left(Z_{V}^{\perp}\right)\right) \Xi_{m}^{u}+\sum_{m \in M}\left(1-\cos \left(\vartheta_{m}\left(Z_{V}^{\perp}\right)\right)\right) \xi_{m}^{u}\right),
\end{aligned}
$$

where $X_{1}^{u}, \xi_{m}^{u}$ and $\Xi_{m}^{u}$ are defined in (IV-U).

For $(4)$, we let $\alpha(s)=\left(\tilde{p}, \cos s\left(\overline{V+Z_{V}^{\perp}}\right)+\sin s \zeta\right)$, so that $a_{1}(s)=\tilde{p}, a_{2}(s)=\cos s\left(\overline{V+Z_{V}^{\perp}}\right)+\sin s \zeta, \dot{a}_{1}(0)=0$ and $\dot{a}_{2}(0)=\zeta$. Here $Z_{0}^{s}=\cos s Z_{V}^{\perp} / \tau+\sin s \zeta$, so that $\vartheta_{m}^{s}=\vartheta_{m}\left(Z_{0}^{s}\right)$ cannot be simplified without additional information on the curves $\vartheta_{m}\left(Z_{0}^{s}\right)$. Note that $\vartheta_{m}^{0}=\frac{1}{\tau} \vartheta_{m}\left(Z_{V}^{\perp}\right)$. If $Z_{V}^{\perp} \notin \mathcal{U}$ (see Definition 2.18), then it is possible that $N \neq \emptyset$ and it is also possible that $\vartheta_{m}^{s}-\vartheta_{h}^{s} \rightarrow 0$ as $s \rightarrow 0$. Here $X_{1}^{s}$ represents the component of $\frac{1}{\tau} V$ in $\operatorname{ker} j\left(Z_{0}^{s}\right)$ and $\xi_{m}^{s}$ represents the component of $\frac{1}{\tau} V$ in $W_{m}\left(Z_{0}^{s}\right)$. Also, $\Xi_{m}^{s}=j\left(Z_{0}^{s}\right) \xi_{m}^{s} / \vartheta_{m}^{s}$. Note that since $V \in \operatorname{ker} j\left(Z_{V}^{\perp}\right)$, we must have $\xi_{m}^{0}=0$ for $m \notin N$, thus $M^{\prime}=\emptyset$. Also, $\dot{X}_{1}^{0}+\sum_{n \in N} \dot{\xi}_{n}^{0}+\sum_{m \in M^{\prime \prime}} \dot{\xi}_{m}^{0}=0$ and $\dot{Z}_{0}^{0}=\zeta$. Note that $\xi_{m}^{0}=0$ implies $\dot{\xi}_{m}^{0} \in W_{m}\left(Z_{V}^{\perp}\right)$ for $m \in M^{\prime \prime}$. Plugging 
these into (2.21) we have $\dot{X}^{0}(\tau)=\sum_{m \in M^{\prime \prime}} \tau\left(1-\cos \left(\vartheta_{m}\left(Z_{V}^{\perp}\right)\right)\right) / \vartheta_{m}\left(Z_{V}^{\perp}\right) \dot{\Xi}_{m}^{0}+$ $\sum_{m \in M^{\prime \prime}} \tau\left(\frac{\sin \left(\vartheta_{m}\left(Z_{V}^{\perp}\right)\right)}{\vartheta_{m}\left(Z_{V}^{\perp}\right)}-1\right) \dot{\xi}_{m}^{0}+\left.\sum_{n \in N} \frac{\tau^{2}}{2} \frac{d}{d s}\right|_{0}\left(J_{s} \xi_{n}^{s}\right)$ and $-\dot{X}^{\prime 0}(\tau)+\dot{X}_{0}^{0}=$ $-\sum_{m \in M^{\prime \prime}} \sin \left(\vartheta_{m}\left(Z_{V}^{\perp}\right)\right) \dot{\Xi}_{m}^{0}+\sum_{m \in M^{\prime \prime}}\left(1-\cos \left(\vartheta_{m}\left(Z_{V}^{\perp}\right)\right)\right) \dot{\xi}_{m}^{0}-\left.\sum_{n \in N} \tau \frac{d}{d s}\right|_{0}\left(J_{s} \xi_{n}^{s}\right)$ and $\dot{Z}^{0}(\tau)=\tau \zeta+[V, B]$ where

$$
\begin{aligned}
B= & \sum_{m \in M^{\prime \prime}} \tau \frac{2 \sin \left(\vartheta_{m}\left(Z_{V}^{\perp}\right)\right)-\vartheta_{m}\left(Z_{V}^{\perp}\right)\left(\cos \left(\vartheta_{m}\left(Z_{V}^{\perp}\right)\right)+1\right)}{2 \vartheta_{m}\left(Z_{V}^{\perp}\right)^{2}} \dot{\Xi}_{m}^{0} \\
& +\sum_{m \in M^{\prime \prime}} \tau \frac{2\left(\cos \left(\vartheta_{m}\left(Z_{V}^{\perp}\right)\right)-1\right)+\vartheta_{m}\left(Z_{V}^{\perp}\right) \sin \left(\vartheta_{m}\left(Z_{V}^{\perp}\right)\right)}{2 \vartheta_{m}\left(Z_{V}^{\perp}\right)^{2}} \dot{\xi}_{m}^{0} \\
& +\left.\sum_{n \in N} \frac{\tau^{2}}{12} \frac{d}{d s}\right|_{0}\left(J_{s} \xi_{n}^{s}\right) .
\end{aligned}
$$

Thus

$$
\begin{aligned}
\left(\operatorname{Id}-\tilde{\Phi}_{\tau *_{\tilde{p}}}\right)(0, \zeta)= & \left(\sum_{m \in M^{\prime \prime}} \tau \frac{\cos \left(\vartheta_{m}\left(Z_{V}^{\perp}\right)\right)-1}{\vartheta_{m}\left(Z_{V}^{\perp}\right)} \dot{\Xi}_{m}^{0}\right. \\
& +\sum_{m \in M^{\prime \prime}} \tau\left(1-\frac{\sin \left(\vartheta_{m}\left(Z_{V}^{\perp}\right)\right)}{\vartheta_{m}\left(Z_{V}^{\perp}\right)}\right) \dot{\xi}_{m}^{0} \\
& -\left.\sum_{n \in N} \frac{\tau^{2}}{2} \frac{d}{d s}\right|_{0}\left(J_{s} \xi_{n}^{s}\right)-\tau \zeta+\frac{\tau}{2}\left[V, \dot{X}_{1}^{0}+\sum_{n \in N} \dot{\xi}_{n}^{0}\right] \\
& +\left[V, \tau \sum_{m \in M^{\prime \prime}} \frac{-\sin \left(\vartheta_{m}\left(Z_{V}^{\perp}\right)\right)+\vartheta_{m}\left(Z_{V}^{\perp}\right)}{\vartheta_{m}\left(Z_{V}^{\perp}\right)^{2}}\right] \\
& +\left[V, \sum_{m \in M^{\prime \prime}} \tau \frac{\left(1-\cos \left(\vartheta_{m}\left(Z_{V}^{\perp}\right)\right)\right)}{\vartheta_{m}\left(Z_{V}^{\perp}\right)^{2}} \dot{\xi}_{m}^{0}+\left.\sum_{n \in N} \frac{\tau^{2}}{6} \frac{d}{d s}\right|_{0}\left(J_{s} \xi_{n}^{s}\right)\right] \\
& -\sum_{m \in M^{\prime \prime}} \sin \left(\vartheta_{m}\left(Z_{V}^{\perp}\right)\right) \dot{\Xi}_{m}^{0}+\sum_{m \in M^{\prime \prime}}\left(1-\cos \left(\vartheta_{m}\left(Z_{V}^{\perp}\right)\right)\right) \dot{\xi}_{m}^{0} \\
& \left.-\left.\sum_{n \in N} \tau \frac{d}{d s}\right|_{0}\left(J_{s} \xi_{n}^{s}\right)\right) .
\end{aligned}
$$

Case IV: The DG-Multiplier. Let $\left\{\bar{V}, \xi_{0}^{1}, \ldots, \xi_{0}^{a_{1}}\right\}$ be an orthonormal basis of $\operatorname{ker} j\left(Z_{V}^{\perp}\right)$. For $m \in \mathcal{M}$ let $\xi_{m}^{1}$ be a unit vector of $W_{m}\left(Z_{V}^{\perp}\right)$. Let $\Xi_{m}^{1}=$ $j\left(Z_{V}^{\perp}\right) \xi_{m}^{1} / \vartheta_{m}\left(Z_{V}^{\perp}\right)$. One easily checks that $\xi_{m}^{1}$ and $\Xi_{m}^{1}$ are orthogonal unit vectors in $W_{m}\left(Z_{V}^{\perp}\right)$. Let $\xi_{m}^{2}$ be a unit vector in $W_{m}\left(Z_{V}^{\perp}\right)$ that is orthogonal 
to both $\xi_{m}^{1}$ and $\Xi_{m}^{1}$. Proceed until we have $\left\{\xi_{m}^{1}, \Xi_{m}^{1}, \xi_{m}^{2}, \Xi_{m}^{2}, \ldots, \xi_{m}^{a_{m}}, \Xi_{m}^{a_{m}}\right\}$, an orthonormal basis of $W_{m}\left(Z_{V}^{\perp}\right)$ such that $\Xi_{m}^{\alpha}=j\left(Z_{V}^{\perp}\right) \xi_{m}^{\alpha} / \vartheta_{m}\left(Z_{V}^{\perp}\right), \alpha=$ $1, \ldots, a_{m}$. We use the convention that if $m \notin \mathcal{M}, \xi_{m}^{\alpha}=0$ and $\Xi_{m}^{\alpha}=0$. Let $\left\{\bar{Z}_{V}^{\perp}, \zeta_{1}, \ldots, \zeta_{c}\right\}$ be an orthonormal basis of $\mathfrak{z}$ such that $\left\{\zeta_{1}, \ldots, \zeta_{b}\right\}$ is a basis of $[V, \mathfrak{g}]$. Now, it is possible that $\xi_{m}^{\alpha} \in C(V, \mathfrak{g})$ and $\Xi_{m}^{\alpha} \notin C(V, \mathfrak{g})$. Thus, for $m \in \mathcal{M}$ and $\alpha=1, \ldots, a_{m}$ we let $\xi_{m}^{\alpha, \perp}$ denote the projection of $\xi_{m}^{\alpha}$ onto $C(V, \mathfrak{g})^{\perp}$, the orthogonal complement of $C(V, \mathfrak{g})$ in $\mathfrak{g}$. Define $\Xi_{m}^{\alpha, \perp}$ analogously. We will need a second basis of $\mathfrak{v}$ as follows. Let $Y_{1}^{\perp}, \ldots, Y_{b}^{\perp}$ be an orthonormal basis of $C(V, \mathfrak{g})^{\perp}$ and let $\bar{V}, Y_{1}, \ldots, Y_{d}$ be an orthonormal basis of $\mathfrak{v} \cap C(V, \mathfrak{g})$ such that $Y_{\delta} \perp v, \delta=1, \ldots, d$.

We set

$$
\mathcal{E}=\left\{\left(Y_{1}, 0\right), \ldots,\left(Y_{d}, 0\right),(v, 0),\left(v^{\perp}, 0\right),\left(\zeta_{1}, 0\right), \ldots,\left(\zeta_{c}, 0\right),(0, v)\right\} .
$$

By Theorem 2.20

$$
\mathcal{F}=\left\{\left(0, Y_{1}\right), \ldots,\left(0, Y_{d}\right),(0, v),\left(0, v^{\perp}\right),\left(0, \zeta_{1}\right), \ldots,\left(0, \zeta_{c}\right),(-v, 0)\right\} .
$$

We set

$$
\mathcal{V}=\left\{\left(Y_{1}^{\perp}, 0\right), \ldots,\left(Y_{b}^{\perp}, 0\right),\left(0, \xi_{0}^{1}\right), \ldots,\left(0, \Xi_{\mu}^{a_{\mu}}\right),\left(0, v^{\perp}\right),\left(0, \zeta_{1}\right), \ldots,\left(0, \zeta_{c}\right)\right\}
$$

Recall that $T=I-\tilde{\Phi}_{\tau *_{\tilde{p}}}$.

Now $T\left(Y_{\beta}^{\perp}, 0\right)=\left(\left[V, Y_{\beta}^{\perp}\right], 0\right)$. Let $M$ be a matrix whose entries are defined by

$$
M_{\delta, \beta}=<\zeta_{\beta},\left[V, Y_{\delta}^{\perp}\right]>
$$

$\beta, \delta=1, \ldots, b$. So

$$
T\left(Y_{1}^{\perp}, 0\right) \wedge \cdots \wedge T\left(Y_{b}^{\perp}, 0\right)= \pm \operatorname{det} M\left(\zeta_{1}, 0\right) \wedge \cdots \wedge\left(\zeta_{b}, 0\right) .
$$

We may now omit elements in $[V, \mathfrak{g}]$ in the left hand component in the remaining values. By $(\mathrm{IV}-2) T\left(0, v^{\perp}\right)=\left(-\tau v^{\perp}, 0\right)$ and by $(\mathrm{IV}-4) T\left(0, \zeta_{\delta}\right)=$ $\left(-\tau \zeta_{\delta}, 0\right)$ for $\delta=b+1, \ldots, c$. To see this note that $\zeta_{\delta} \perp[V, \mathfrak{g}]$, which implies $X_{1}^{s}=V / \tau$ and $X_{2}^{s}=0$ when applying $(\mathrm{IV}-4)$. So

$$
\begin{aligned}
& T\left(0, v^{\perp}\right) \wedge T\left(0, \zeta_{b+1}\right) \wedge \cdots \wedge T\left(0, \zeta_{c}\right)= \pm \tau^{c-b+1}\left(v^{\perp}, 0\right) \\
& \wedge\left(\zeta_{b+1}, 0\right) \wedge \cdots \wedge\left(\zeta_{c}, 0\right) .
\end{aligned}
$$

By $(\mathrm{IV}-3) T\left(0, \xi_{0}^{\alpha}\right)=\left(-\tau \xi_{0}^{\alpha}+[V, \star], 0\right)$, so the contribution of $T\left(0, \xi_{0}^{1}\right)$ $\wedge \cdots \wedge T\left(0, \xi_{0}^{a_{0}}\right)$ to $T \mathcal{V} \wedge \mathcal{F}$ is $\pm \tau^{a_{0}}\left(\xi_{0}^{1}, 0\right) \wedge \cdots \wedge\left(\xi_{0}^{a_{0}}, 0\right)$. For $m \in \mathcal{M}$ we 
have

$$
\begin{aligned}
& T\left(0, \xi_{m}^{\alpha}\right) \\
& =\left(-s_{m} \tau \xi_{m}^{\alpha}-c_{m} \tau \Xi_{m}^{\alpha}+[V, \star],\left(1-\cos \left(\tau \vartheta_{m}\left(Z_{V}^{\perp}\right)\right) \xi_{m}^{\alpha}-\sin \left(\tau \vartheta_{m}\left(Z_{V}^{\perp}\right)\right) \Xi_{m}^{\alpha}\right)\right.
\end{aligned}
$$

and

$$
\begin{aligned}
& T\left(0, \Xi_{m}^{\alpha}\right) \\
& =\left(c_{m} \tau \xi_{m}^{\alpha}-s_{m} \tau \Xi_{m}^{\alpha}+[V, \star], \sin \left(\tau \vartheta_{m}\left(Z_{V}^{\perp}\right)\right) \xi_{m}^{\alpha}+\left(1-\cos \left(\tau \vartheta_{m}\left(Z_{V}^{\perp}\right)\right)\right) \Xi_{m}^{\alpha}\right),
\end{aligned}
$$

where $c_{m}=\left(1-\cos \left(\tau \vartheta_{m}\left(Z_{V}^{\perp}\right)\right)\right) / \vartheta_{m}\left(Z_{V}^{\perp}\right)$ and $s_{m}=\sin \left(\tau \vartheta_{m}\left(Z_{V}^{\perp}\right)\right) / \vartheta_{m}\left(Z_{V}^{\perp}\right)$.

The contribution of $\mathcal{F}$ means that $\left(0, Y_{1}\right) \wedge \cdots \wedge\left(0, Y_{d}\right)$ is wedged with every term in $T \mathcal{V} \wedge \mathcal{F}$. Thus elements in $\mathfrak{v}$ in the right-hand components may be projected onto $C(V, \mathfrak{g})^{\perp}$, and we may ignore further values $[V, \star]$ as they contribute nothing to $T \mathcal{V} \wedge \mathcal{F}$. Likewise, we may replace $\xi_{m}^{\alpha}$ and $\Xi_{m}^{\alpha}$ with $\xi_{m}^{\alpha, \perp}$ and $\Xi_{m}^{\alpha, \perp}$ in the right-hand component. Thus, the contribution of $T\left(0, \xi_{m}^{\alpha}\right) \wedge T\left(0, \Xi_{m}^{\alpha}\right)$ to $T \mathcal{V} \wedge \mathcal{F}$ is

$$
\pm \frac{2\left(1-\cos \left(\tau \vartheta_{m}\left(Z_{V}^{\perp}\right)\right)\right) \tau^{2}}{\vartheta_{m}\left(Z_{V}^{\perp}\right)^{2}}\left(\xi_{m}^{\alpha}, \frac{\vartheta_{m}\left(Z_{V}^{\perp}\right)}{\tau} \Xi_{m}^{\alpha, \perp}\right) \wedge\left(\Xi_{m}^{\alpha}, \frac{-\vartheta_{m}\left(Z_{V}^{\perp}\right)}{\tau} \xi_{m}^{\alpha, \perp}\right)
$$

Now,

$$
\begin{aligned}
T\left(0, \zeta_{\beta}\right) & =\left(\tau \sum_{m \in M}\left(\left(1-s_{m}\right) \dot{\xi}_{m, \beta}^{0}-c_{m} \dot{\Xi}_{m, \beta}^{0}\right)-\tau \zeta_{\beta}+[V, \star]\right. \\
& -\left.\sum_{n \in N} \frac{\tau^{2}}{2} \frac{d}{d s}\right|_{0}\left(J_{s} \xi_{n, \beta}^{s}\right), \sum_{m \in M}\left(1-\cos \left(\vartheta_{m}\left(Z_{V}^{\perp}\right)\right)\right) \dot{\xi}_{m, \beta}^{0} \\
& \left.-\sin \left(\vartheta_{m}\left(Z_{V}^{\perp}\right)\right) \dot{\Xi}_{m, \beta}^{0}-\left.\sum_{n \in N} \tau \frac{d}{d s}\right|_{\left.\right|_{0}}\left(J_{s} \xi_{n, \beta}^{s}\right)\right)
\end{aligned}
$$

where $\xi_{m, \beta}^{s}$ is the component of $\frac{1}{\tau} V$ in $W_{m}\left(\cos s \frac{1}{\tau} Z_{V}^{\perp}+\sin s \zeta_{\beta}\right)$ and $\dot{\xi}_{m, \beta}=$ $\left.\frac{d}{d s}\right|_{0} \xi_{m, \beta}^{s}$ and $\dot{\Xi}_{m, \beta}=j\left(Z_{V}^{\perp}\right) \dot{\xi}_{m, \beta} / \vartheta_{m}\left(Z_{V}^{\perp}\right)$. As $\xi_{m, \beta}^{0}=0$ for all $m, \beta$, we have $\dot{\xi}_{m, \beta}^{0}, \dot{\Xi}_{m, \beta}^{0} \in W_{m}\left(Z_{V}^{\perp}\right)$ for all $m \in M$. By writing $\dot{\xi}_{m, \beta}^{0}$ and $\dot{\Xi}_{m, \beta}^{0}$ as linear combinations of the $\xi_{m}^{\alpha}$ and $\Xi_{m}^{\alpha}$, and by row reducing with the elements in $T\left(0, \xi_{m}^{\alpha}\right) \wedge T\left(0, \Xi_{m}^{\alpha}\right)$, above, we see that the contribution of $T\left(0, \zeta_{\beta}\right)$ to $T \mathcal{V} \wedge \mathcal{F}$ is

$$
\left(\tau \sum_{m \in M} \dot{\xi}_{m, \beta}^{0}-\left.\sum_{n \in N} \frac{\tau^{2}}{2} \frac{d}{d s}\right|_{\left.\right|_{0}}\left(J_{s} \xi_{n, \beta}^{s}\right),-\left.\sum_{n \in N} \tau \frac{d}{d s}\right|_{\left.\right|_{0}}\left(J_{s} \xi_{n, \beta}^{s}\right)\right)
$$


Let $U_{\beta}=\sum_{m \in M} \dot{\xi}_{m, \beta}^{0}$ and $J_{\beta}=\left.\sum_{n \in N} \frac{d}{d s}\right|_{0} J_{s} \xi_{n, \beta}^{s}$.

We now use the fact that both $\left\{\bar{V}, \xi_{0}^{1}, \ldots, \Xi_{\mu}^{a_{\mu}}\right\}$ and $\left\{\bar{V}, Y_{1}^{\perp}, \ldots, Y_{b}^{\perp}\right.$, $\left.Y_{1}, \ldots, Y_{d}\right\}$ are orthonormal bases of $\mathfrak{v}$. Let $\hat{M}$ be the matrix whose $\beta-\delta$ entry is

$$
\begin{aligned}
\hat{M}_{\beta, \delta}= & \sum_{m \in M} \sum_{\alpha=1}^{a_{m}}\left(\left\langle-\tau J_{\beta}, Y_{\delta}^{\perp}\right\rangle-\left\langle\frac{\vartheta_{m}}{\tau} \Xi_{m}^{\alpha, \perp}, Y_{\delta}^{\perp}\right\rangle\left\langle\tau U_{\beta}-\frac{\tau^{2}}{2} J_{\beta}, \xi_{m}^{\alpha}\right\rangle\right. \\
(\mathrm{IV}-\hat{M}) & \left.+\left\langle\frac{\vartheta_{m}}{\tau} \xi_{m}^{\alpha, \perp}, Y_{\delta}^{\perp}\right\rangle\left\langle\tau U_{\beta}-\frac{\tau^{2}}{2} J_{\beta}, \Xi_{m}^{\alpha}\right\rangle\right),
\end{aligned}
$$

$\beta, \delta=1, \ldots, b$. Then, putting everything above together, we obtain a DG-multiplier of

$$
|\operatorname{det} M \operatorname{det} \hat{M}|^{-1 / 2} \tau^{-(\operatorname{dim} \mathfrak{g}-\operatorname{dim}[V, \mathfrak{g}]-1) / 2} \times
$$

$$
\prod_{m \in \mathcal{M}\left(Z_{V}^{\perp}\right)}\left(\frac{2\left(1-\cos \left(\vartheta_{m}\left(Z_{V}^{\perp}\right)\right)\right.}{\vartheta_{m}\left(Z_{V}^{\perp}\right)^{2}}\right)^{-\operatorname{dim} W_{m}\left(Z_{V}^{\perp}\right) / 4}
$$

where $M$ and $\hat{M}$ are defined in $(\mathrm{IV}-M)$ and $(\mathrm{IV}-\hat{M})$, above.

Note that the values of $\hat{M}, U_{\beta}, J_{\beta}$ depend on the chosen curves, but the final expression (IV-DG) will not.

Case V: Poincaré Map. $\gamma=\exp \left(V+Z_{V}+Z_{V}^{\perp}\right), V \neq 0, Z_{V}^{\perp} \neq 0$ and $\tau<$ $\left|V+Z_{V}^{\perp}\right|$.

By Theorem 2.11, the periods $\tau$ of $\gamma$ with $\tau<\left|V+Z_{V}^{\perp}\right|$ are precisely

$$
\left\{\sqrt{|V|^{2}+\frac{\left|Z_{V}^{\perp}\right|^{2}}{\left|K_{V}^{\perp}\left(X_{2}+Z_{0}\right)\right|^{2}}}: X_{2}+Z_{0} \text { satisfy (i)-(iv) below }\right\} .
$$

Given $Z_{0} \in \mathfrak{z}$ and $X_{2}=\sum_{m \in \mathcal{M}} \xi_{m} \in \mathfrak{v}$, where $\xi_{m} \in W_{m}\left(Z_{0}\right)$, the conditions referred to above are the following:

(i) $\left|X_{2}+Z_{0}\right|=1$

(ii) $V \in \operatorname{ker} j\left(Z_{0}\right)$ and $X_{2} \perp \operatorname{ker} j\left(Z_{0}\right), X_{2} \neq 0$

(iii) $Z_{V}^{\perp} \in \operatorname{span}_{\mathbb{R}^{+}}\left\{K_{V}^{\perp}\left(X_{2}+Z_{0}\right)\right\}$

(iv) for all $m \in \mathcal{M}\left(Z_{0}\right)$ such that $\xi_{m} \neq 0, \frac{\left|Z_{V}^{\perp}\right| \vartheta_{m}\left(Z_{0}\right)}{2 \pi\left|K_{V}^{\perp}\left(X_{2}+Z_{0}\right)\right|} \in \mathbb{Z}$. 
Let $\tau_{2}=\frac{|Z|}{\left|K_{V}^{\perp}\left(\hat{X}_{0}+\hat{Z}_{0}\right)\right|}$ where $\hat{X}_{0}+\hat{Z}_{0}$ satisfies (i)-(iv) above. Let $\tau=$ $\sqrt{|V|^{2}+\tau_{2}^{2}}$. Then by Theorem 2.12

$$
\begin{aligned}
\tilde{F}(\tau, \gamma)= & \left\{v_{\tilde{p}}: v=\frac{1}{\tau}\left(V+\tau_{2}\left(X_{2}+Z_{0}\right)\right), \tilde{p} \quad\right. \text { and } \\
& \left.X_{2}+Z_{0} \text { satisfy (a)-(e) below }\right\} .
\end{aligned}
$$

Given $\tilde{p} \in G, Z_{0} \in \mathfrak{z}$ and $X_{2}=\sum_{m \in \mathcal{M}} \xi_{m} \in \mathfrak{v}$, where $\xi_{m} \in W_{m}\left(Z_{0}\right)$, the conditions referred to above are the following:

(a) $\left|X_{2}+Z_{0}\right|=1$,

(b) $V \in \operatorname{ker} j\left(Z_{0}\right)$ and $X_{2} \perp \operatorname{ker} j\left(Z_{0}\right), X_{2} \neq 0$,

(c) $K_{V}^{\perp}\left(X_{2}+Z_{0}\right)=\frac{1}{\tau_{2}} Z_{V}^{\perp}$,

(d) for all $m \in \mathcal{M}\left(Z_{0}\right)$ such that $\xi_{m} \neq 0, \frac{\tau_{2}}{2 \pi} \vartheta_{m}\left(Z_{0}\right) \in \mathbb{Z}$ and

(e) $[V, \log \tilde{p}]+Z_{V}=\left[V, j\left(Z_{0}\right)^{-1} X_{2}\right]+\tau_{2} K_{V}\left(X_{2}+Z_{0}\right)$.

Let $v_{\tilde{p}} \in \tilde{F}(\tau, \gamma)$, so $v_{\tilde{p}}=\left(\tilde{p}, X_{0}+Z_{0}\right)$ where $v=\frac{1}{\tau}\left(V+\tau_{2}\left(X_{2}+Z_{0}\right)\right)$ where $\tilde{p}$ and $X_{2}+Z_{0}$ satisfy $(a)-(e)$ above. Note that $\tilde{p}=x \tilde{q} a$, where $x \in C(\gamma, G), \tilde{q}^{-1} \gamma \tilde{q}=\exp \left(V+Z_{V}^{\perp}\right)$ and $a \in G$. Also note by condition (e) that if $(x \tilde{q} a, v) \in \tilde{F}(\tau, \gamma)$, then $(y \tilde{q} a, v) \in \tilde{F}(\tau, \gamma)$ for all $y \in C(\gamma, G)$, thus $C(\log \gamma, \mathfrak{g}) \times\{0\} \subset T_{v_{\tilde{p}}} \tilde{F}(\tau, \gamma)$. (See Section 4.2 for an example where this containment is proper.) So $\tilde{\Phi}_{\tau *_{v}}=\mathrm{Id}$ when restricted to $C(\log \gamma, \mathfrak{g}) \times\{0\}$. We must calculate $(1) \tilde{\Phi}_{\tau *_{\tilde{p}}}(U, 0)$ where $U \perp C(\log \gamma, \mathfrak{g}),(2) \tilde{\Phi}_{\tau *_{\tilde{p}}}\left(0, v_{1}^{\perp}\right)$ where $v_{1}^{\perp} \in \operatorname{span}_{\mathbb{R}}\left\{X_{2}, Z_{0}\right\}, v_{1}^{\perp} \perp v,(3) \tilde{\Phi}_{\tau *_{\tilde{p}}}\left(0, v_{2}^{\perp}\right)$ where $v_{2}^{\perp} \in \operatorname{span}_{\mathbb{R}}\{V$, $\left.X_{2}, Z_{0}\right\}, v_{2}^{\perp} \perp v, v_{2}^{\perp} \not \perp V$, (4) $\tilde{\Phi}_{\tau *_{\tilde{p}}}(0, U)$ where $U \in \mathfrak{v}, U \perp \operatorname{span}\left\{v, X_{2}\right\}$ and (5) $\tilde{\Phi}_{\tau *_{\tilde{p}}}(0, \zeta)$ where $\zeta \in \mathfrak{z}, \zeta \perp v$.

For $(1)$, we let $\alpha(s)=(\tilde{p} \exp (s U), v)$, so $a_{1}(s)=s U, a_{2}(s)=v, \dot{a}_{1}(0)=$ $U$, and $\dot{a}_{2}(0)=0$. Recall that $X_{2}=\sum_{m \in \mathcal{M}} \xi_{m}$, where $\xi_{m} \in W_{m}\left(Z_{0}\right)$. Then $X_{1}^{s}=V / \tau, X_{2}^{s}=\frac{\tau_{2}}{\tau} X_{2}, Z_{0}^{s}=\frac{\tau_{2}}{\tau} Z_{0}$ and $\vartheta_{m}^{s}=\vartheta_{m}\left(Z_{0}^{s}\right)=\frac{\tau_{2}}{\tau} \vartheta_{m}\left(Z_{0}\right)$. So $\dot{X}_{1}^{0}=$ $0, \dot{X}_{2}^{0}=0, \dot{\xi}_{m}^{0}=0, \dot{\Xi}_{m}^{0}=0, \dot{\vartheta}_{m}^{0}=0$ and $\dot{Z}_{0}^{0}=0$ for $m \in \mathcal{M}$. Also, $\vartheta_{m}^{0}=0$ if and only if $\vartheta_{m}\left(Z_{0}\right)=0$, so $N=\emptyset$, and by $(2.21)$ we have $\dot{X}^{0}(\tau)=0$, $-\dot{X}^{\prime} 0(\tau)+\dot{X}_{0}^{0}=0$ and $\dot{Z}^{0}(\tau)=0$. Thus by $(2.18)$

$$
\left(\mathrm{Id}-\tilde{\Phi}_{\tau *_{\tilde{p}}}\right)(U, 0)=([V, U], 0)
$$

For (2), let $\bar{X}_{2}=X_{2} /\left|X_{2}\right|$ and $\bar{Z}_{0}=Z_{0} /\left|Z_{0}\right|$. As $\left|X_{2}+Z_{0}\right|=1$, there exists $\beta_{1}$ so that $X_{2}+Z_{0}=\cos \beta_{1} \bar{X}_{2}+\sin \beta_{1} \bar{Z}_{0}$. That is, $\cos \beta_{1}=\left|X_{2}\right|$ and $\sin \beta_{1}=\left|Z_{0}\right|$. Recall that $X_{2}=\sum_{m \in \mathcal{M}} \xi_{m}$ where $\xi_{m} \in W_{m}\left(Z_{0}\right)$, and let 
$\bar{X}_{2}=\sum_{m \in \mathcal{M}} \bar{\xi}_{m}$, where $\bar{\xi}_{m} \in W_{m}\left(Z_{0}\right)$, so $\bar{\xi}_{m}=\xi_{m} /\left|X_{2}\right|$. Let $\bar{\vartheta}_{m}=\vartheta_{m}\left(\bar{Z}_{0}\right)$ and let $\bar{J}=j\left(\bar{Z}_{0}\right)$. Let $\bar{\Xi}_{m}=\bar{J} \xi_{m} / \bar{\vartheta}_{m}$. Set $v_{1}^{\perp}=-\sin \beta_{1} \bar{X}_{2}+\cos \beta_{1} \bar{Z}_{0}$. Now let $\alpha(s)=\left(\tilde{p}, \frac{1}{\tau} V+\frac{\tau_{2}}{\tau}\left(\cos \left(\beta_{1}+s\right) \bar{X}_{2}+\sin \left(\beta_{1}+s\right) \bar{Z}_{0}\right)\right)$, so that $a_{1}(s)=$ $e, a_{2}(s)=\frac{1}{\tau} V+\frac{\tau_{2}}{\tau}\left(\cos \left(\beta_{1}+s\right) \bar{X}_{2}+\sin \left(\beta_{1}+s\right) \bar{Z}_{0}\right), \dot{a}_{1}(0)=0$ and $\dot{a}_{2}(0)=$ $\frac{\tau_{2}}{\tau} v_{1}^{\perp}$. Here $Z_{0}^{s}=\frac{\tau_{2}}{\tau} \sin \left(\beta_{1}+s\right) \bar{Z}_{0}$, so $\vartheta_{m}^{s}=\vartheta_{m}\left(Z_{0}^{s}\right)=\frac{\tau_{2}}{\tau} \sin \left(\beta_{1}+s\right) \bar{\vartheta}_{m}$, and $\dot{\vartheta}_{m}^{0}=\frac{\tau_{2}}{\tau} \cos \beta_{1} \bar{\vartheta}_{m}$ for $m \in \mathcal{M}$. Also, as $\sin \beta_{1} \neq 0, \vartheta_{m}^{0}=0$ if and only if $\bar{\vartheta}_{m}=0$, so $N=\emptyset$ and $M=\mathcal{M}$. Then $X_{1}^{s}=V / \tau, X_{2}^{s}=\frac{\tau_{2}}{\tau} \cos \left(\beta_{1}+s\right) \bar{X}_{2}$ so that $\xi_{m}^{s}=\frac{\tau_{2}}{\tau} \cos \left(\beta_{1}+s\right) \bar{\xi}_{m}$ and $\Xi_{m}^{s}=\frac{\tau_{2}}{\tau} \cos \left(\beta_{1}+s\right) \bar{\Xi}_{m}$. From this we have $X_{1}^{0}=0, \xi_{m}^{0}=\frac{\tau_{2}}{\tau} \cos \beta_{1} \bar{\xi}_{m}, \Xi_{m}^{0}=\frac{\tau_{2}}{\tau} \cos \beta_{1} \bar{\Xi}_{m}, \dot{Z}_{0}^{0}=\frac{\tau_{2}}{\tau} \cos \beta_{1} \bar{Z}_{0}$ and $\dot{X}_{1}^{0}=$ 0. Also, $\dot{\xi}_{m}^{0}=-\frac{\tau_{2}}{\tau} \sin \beta_{1} \bar{\xi}_{m}$ and $\dot{\Xi}_{m}^{0}=-\frac{\tau_{2}}{\tau} \sin \beta_{1} \bar{\Xi}_{m}$. This implies that if $\xi_{m}^{0}=0$ then $\dot{\xi}_{m}^{0}=0$, so that the terms with $m \in M^{\prime \prime}$ contribute nothing to the following calculations. Plugging these into $(2.21)$ we have $\dot{X}^{0}(\tau)=$ $\tau_{2} \cos \beta_{1} \cot \beta_{1} \bar{X}_{2},-\dot{X}^{\prime} 0(\tau)+\dot{X}_{0}^{0}=-\frac{\tau_{2}^{2}}{\tau} \cos ^{2} \beta_{1} \bar{J}_{2}$ and

$$
\begin{aligned}
\dot{Z}^{0}(\tau)= & \tau_{2} \cos \beta_{1} \bar{Z}_{0}+\left[V,-\left(2 \cot ^{2} \beta_{1}+1\right) \bar{J}^{-1} \bar{X}_{2}+\frac{\tau_{2}}{2} \cot \beta_{1} \cos \beta_{1} \bar{X}_{2}\right] \\
& +\tau_{2} \cos \beta_{1} \csc ^{2} \beta_{1} \sum_{m \in \mathcal{M}}\left[\bar{\xi}_{m}, \bar{J}^{-1} \bar{\xi}_{m}\right] .
\end{aligned}
$$

After normalizing by $\tau_{2} / \tau$ and substituting we have

$$
\begin{aligned}
\left(\operatorname{Id}-\tilde{\Phi}_{\tau *_{\tilde{p}}}\right)\left(0, v_{1}^{\perp}\right)= & \left(\operatorname{Id}-\tilde{\Phi}_{\tau *_{\tilde{p}}}\right)\left(0,-\frac{\left|Z_{0}\right|}{\left|X_{2}\right|} X_{2}+\frac{\left|X_{2}\right|}{\left|Z_{0}\right|} Z_{0}\right) \\
= & \left(-\tau \frac{\left|X_{2}\right|}{\left|Z_{0}\right|} X_{2}-\tau \frac{\left|X_{2}\right|}{\left|Z_{0}\right|} Z_{0}-\frac{\tau}{\left|X_{2}\right|\left|Z_{0}\right|} \sum_{m \in \mathcal{M}}\left[\xi_{m}, J^{-1} \xi_{m}\right]\right. \\
(\mathrm{V}-2) \quad & \left.+\frac{\tau}{\tau_{2}} \frac{\left(1+\left|X_{2}\right|^{2}\right)}{\left|X_{2}\right|\left|Z_{0}\right|}\left[V, J^{-1} X_{2}\right],-\tau_{2} \frac{\left|X_{2}\right|}{\left|Z_{0}\right|} J X_{2}\right)
\end{aligned}
$$

For (3), let $\bar{V}=V /|V|$. As $|v|=1$, there exists $\beta_{2}$ so that $v=\cos \beta_{2} \bar{V}+$ $\sin \beta_{2}\left(X_{2}+Z_{0}\right)$. In particular, $\cos \beta_{2}=|V| / \tau$ and $\sin \beta_{2}=\frac{\tau_{2}}{\tau}$. Recall that $X_{2}=\sum_{m \in \mathcal{M}} \xi_{m}$, where $\xi_{m} \in W_{m}\left(Z_{0}\right)$. Let $\vartheta_{m}=\vartheta_{m}\left(Z_{0}\right)$ and let $J=j\left(Z_{0}\right)$. Let $\Xi_{m}=J \xi_{m} / \vartheta_{m}$. Set $v_{2}^{\perp}=-\sin \beta_{2} \bar{V}+\cos \beta_{2}\left(X_{2}+Z_{0}\right)$. Now let $\alpha(s)=$ $\left(\tilde{p}, \cos \left(\beta_{2}+s\right) \bar{V}+\sin \left(\beta_{2}+s\right)\left(X_{2}+Z_{0}\right)\right), \quad$ so that $a_{1}(s)=e, \quad a_{2}(s)=$ $\cos \left(\beta_{2}+s\right) \bar{V}+\sin \left(\beta_{2}+s\right)\left(X_{2}+Z_{0}\right), \dot{a}_{1}(0)=0$ and $\dot{a}_{2}(0)=v_{2}^{\perp}$. Here $Z_{0}^{s}=$ $\sin \left(\beta_{2}+s\right) \bar{Z}_{0}$, so $\vartheta_{m}^{s}=\vartheta_{m}\left(Z_{0}^{s}\right)=\sin \left(\beta_{2}+s\right) \vartheta_{m}$, and $\dot{\vartheta}_{m}^{0}=\cos \beta_{2} \vartheta_{m}$ for $m \in$ $\mathcal{M}$. Also, as $\sin \beta_{2} \neq 0, \vartheta_{m}^{0}=0$ if and only if $\vartheta_{m}=0$, so $N=\emptyset$ and $M=\mathcal{M}$. Then $X_{1}^{s}=\cos \left(\beta_{2}+s\right) \bar{V}, X_{2}^{s}=\sin \left(\beta_{2}+s\right) X_{2}$ so that $\xi_{m}^{s}=\sin \left(\beta_{2}+s\right) \xi_{m}$ and $\Xi_{m}^{s}=\sin \left(\beta_{2}+s\right) \Xi_{m}$. From this we have $X_{1}^{0}=\cos \beta_{2} \bar{V}, \xi_{m}^{0}=\sin \beta_{2} \xi_{m}$, $\Xi_{m}^{0}=\sin \beta_{2} \Xi_{m}, \dot{Z}_{0}^{0}=\cos \beta_{2} Z_{0}$ and $\dot{X}_{1}^{0}=-\sin \beta_{2} \bar{V}$. Also, $\dot{\xi}_{m}^{0}=\cos \beta_{2} \xi_{m}$ and $\dot{\Xi}_{m}^{0}=\cos \beta_{2} \Xi_{m}$. This implies that if $\xi_{m}^{0}=0$ then $\dot{\xi}_{m}^{0}=0$, so that the 
terms with $m \in M^{\prime \prime}$ contribute nothing to the following calculations. Plugging these into $(2.21)$ we have $\dot{X}^{0}(\tau)=-\tau \sin \beta_{2} \bar{V}+\tau \cos \beta_{2} X_{2},-\dot{X}^{\prime 0}(\tau)+$ $\dot{X}_{0}^{0}=-\tau \sin \beta_{2} \cos \beta_{2} J X_{2} \quad$ and $\quad \dot{Z}^{0}(\tau)=\tau \cos \beta_{2} Z_{0}-\left(\tau \sin \beta_{2} /|V|+\right.$ $\left.\cot \beta_{2}\right)\left[V, J^{-1} X_{2}\right]+\frac{\tau \cos \beta_{2}}{2}\left[V, X_{2}\right]$. By $(2.18)$

$$
\begin{aligned}
& \left(\operatorname{Id}-\tilde{\Phi}_{\tau *_{\tilde{p}}}\right)\left(0, v_{2}^{\perp}\right)=\left(\operatorname{Id}-\tilde{\Phi}_{\tau *_{\tilde{p}}}\right)\left(0,-\frac{\tau_{2}}{\tau|V|} V+\frac{|V|}{\tau}\left(X_{2}+Z_{0}\right)\right) \\
& =\left(-\tau v_{2}^{\perp}+\frac{\tau^{2}}{\tau_{2}|V|}\left[V, J^{-1} X_{2}\right],-\frac{\tau_{2}}{\tau}|V| J X_{2}\right)
\end{aligned}
$$

For $(4)$, we let $\alpha(s)=(\tilde{p}, \sin s U+\cos s v)$, so that $a_{1}(s)=e, a_{2}(s)=$ $\sin s U+\cos s v, \dot{a}_{1}(0)=0$ and $\dot{a}_{2}(0)=U$. Recall that $X_{2}=\sum_{m \in M} \xi_{m}$, where $\xi_{m} \in W_{m}\left(Z_{0}\right)$. Let $\vartheta_{m}=\vartheta_{m}\left(Z_{0}\right)$, and let $\Xi_{m}=j\left(Z_{0}\right) \xi_{m} / \vartheta_{m}$. Let

$$
U=X_{1}^{u}+\sum_{m \in M} \xi_{m}^{u} \quad \text { and } \quad \Xi_{m}^{u}=j\left(Z_{0}\right) \xi_{m}^{u} / \vartheta_{m}
$$

where $X_{1}^{u} \in \operatorname{ker} j\left(Z_{0}\right)$ and $\xi_{m}^{u} \in W_{m}\left(Z_{0}\right)$, for $m \in \mathcal{M}\left(Z_{0}\right)$. Here $Z_{0}^{s}=$ $\cos s \frac{\tau_{2}}{\tau} Z_{0}$, so $\vartheta_{m}^{s}=\vartheta_{m}\left(Z_{0}^{s}\right)=\cos s \frac{\tau_{2}}{\tau} \vartheta_{m}$, and $\dot{\vartheta}_{m}^{0}=0$ for $m \in \mathcal{M}$. Also, $\vartheta_{m}^{0}=0$ if and only if $\vartheta_{m}=0$, so $N=\emptyset$ and $M=\mathcal{M}$. Now $X_{1}^{s}=\cos s \frac{\tau_{2}}{\tau^{2}} V+$ $\sin s X_{1}^{u}$ and $X_{2}^{s}=\sum_{m \in \mathcal{M}}\left(\cos s \frac{\tau_{2}}{\tau} \xi_{m}+\sin s \xi_{m}^{u}\right)$ so that $\xi_{m}^{s}=\cos s \frac{\tau_{2}}{\tau} \xi_{m}+$ $\sin s \xi_{m}^{u}$, and $\Xi_{m}^{s}=\cos s \frac{\tau_{2}}{\tau} \Xi_{m}+\sin s \Xi_{m}^{u}$. From this we have $X_{1}^{0}=\frac{1}{\tau} V, X_{2}^{0}=$ $\frac{\tau_{2}}{\tau} X_{2}$ and $\xi_{m}^{0}=\frac{\tau_{2}}{\tau} \xi_{m}, \Xi_{m}^{0}=\frac{\tau_{2}}{\tau} \Xi_{m}, \dot{Z}_{0}^{0}=0, \dot{X}_{1}^{0}=X_{1}^{u}, \dot{\xi}_{m}^{0}=\xi_{m}^{u}$ and $\dot{\Xi}_{m}^{0}=$ $\Xi_{m}^{u}$. Note that $\xi_{m}$ and $\xi_{m}^{u}$ are not, in general, related so that $M^{\prime \prime}$ is a consideration in what follows. Plugging these into $(2.21)$ we have $\dot{X}^{0}(\tau)=$ $\tau X_{1}^{u}+\sum_{m \in \mathcal{M}} \frac{\tau\left(1-\cos \left(\tau_{2} \vartheta_{m}\right)\right)}{\tau_{2} \vartheta_{m}} \Xi_{m}^{u}+\sum_{m \in \mathcal{M}} \frac{\tau \sin \left(\tau_{2} \vartheta_{m}\right)}{\tau_{2} \vartheta_{m}} \xi_{m}^{u}, \quad-\dot{X}^{\prime}(\tau)+\dot{X}_{0}^{0}=$ $-\sum_{m \in \mathcal{M}} \sin \left(\tau_{2} \vartheta_{m}\right) \Xi_{m}^{\tau_{2}}+\sum_{m \in \mathcal{M}}\left(1-\cos \left(\tau_{2} \vartheta_{m}\right)\right)^{u} \xi_{m}^{u}$, and

$$
\begin{aligned}
\dot{Z}^{0}(\tau)= & -\sum_{m \in M^{\prime}} \frac{\tau}{\vartheta_{m}}\left[X_{1}^{u}, \Xi_{m}\right]+\sum_{m \in \mathcal{M}} \frac{\tau}{2 \vartheta_{m}}\left(\left[\xi_{m}^{u}, \Xi_{m}\right]+\left[\xi_{m}, \Xi_{m}^{u}\right]\right) \\
& +\sum_{\substack{m \in M^{\prime \prime}, h \in M^{\prime} \\
m \neq h}} \frac{\tau \vartheta_{h}\left(1-\cos \left(\tau_{2} \vartheta_{m}\right)\right)}{\tau_{2} \vartheta_{m}\left(\vartheta_{m}^{2}-\vartheta_{h}^{2}\right)}\left[\Xi_{m}^{u}, \Xi_{h}\right] \\
& -\sum_{\substack{m \in M^{\prime \prime}, h \in M^{\prime} \\
m \neq h}} \frac{\tau \sin \left(\tau_{2} \vartheta_{m}\right)}{\tau_{2}\left(\vartheta_{m}^{2}-\vartheta_{h}^{2}\right)}\left[\Xi_{m}^{u}, \xi_{h}\right] \\
& +\sum_{\substack{m \in M^{\prime \prime}, h \in M^{\prime} \\
m \neq h}} \frac{\tau \vartheta_{h} \sin \left(\tau_{2} \vartheta_{m}\right)}{\tau_{2} \vartheta_{m}\left(\vartheta_{m}^{2}-\vartheta_{h}^{2}\right)}\left[\xi_{m}^{u}, \Xi_{h}\right]
\end{aligned}
$$




$$
\begin{aligned}
& +\sum_{\substack{m \in M^{\prime \prime}, h \in M^{\prime} \\
m \neq h}} \frac{\tau\left(1-\cos \left(\tau_{2} \vartheta_{m}\right)\right)}{\tau_{2}\left(\vartheta_{m}^{2}-\vartheta_{h}^{2}\right)}\left[\xi_{m}^{u}, \xi_{h}\right] \\
& +\left[V, \sum_{m \in \mathcal{M}} \frac{2 \tau \sin \left(\tau_{2} \vartheta_{m}\right)-\tau_{2} \tau \vartheta_{m}\left(1+\cos \left(\tau_{2} \vartheta_{m}\right)\right)}{2 \tau_{2}^{2} \vartheta_{m}^{2}} \Xi_{m}^{u}\right] \\
& +\left[V, \sum_{m \in \mathcal{M}} \frac{2 \tau\left(\cos \left(\tau_{2} \vartheta_{m}\right)-1\right)+\tau_{2} \tau \vartheta_{m} \sin \left(\tau_{2} \vartheta_{m}\right)}{2 \tau_{2}^{2} \vartheta_{m}^{2}} \xi_{m}^{u}\right]
\end{aligned}
$$

Thus

$$
\begin{aligned}
&(\mathrm{Id}\left.-\tilde{\Phi}_{\tau *_{v_{\tilde{p}}}}\right)(0, U) \\
&=\left(-\tau X_{1}^{u}+\sum_{m \in \mathcal{M}} \frac{\tau\left(\cos \left(\tau_{2} \vartheta_{m}\right)-1\right)}{\tau_{2} \vartheta_{m}} \Xi_{m}^{u}\right. \\
&-\sum_{m \in \mathcal{M}} \frac{\tau \sin \left(\tau_{2} \vartheta_{m}\right)}{\tau_{2} \vartheta_{m}} \xi_{m}^{u}+\sum_{m \in M^{\prime}} \frac{\tau}{\vartheta_{m}}\left[X_{1}^{u}, \Xi_{m}\right] \\
&-\sum_{m \in \mathcal{M}} \frac{\tau}{2 \vartheta_{m}}\left(\left[\xi_{m}^{u}, \Xi_{m}\right]+\left[\xi_{m}, \Xi_{m}^{u}\right]\right) \\
&+\sum_{m \in M^{\prime \prime}, h \in M^{\prime}} \frac{\tau \vartheta_{h}\left(\cos \left(\tau_{2} \vartheta_{m}\right)-1\right)}{\tau_{2} \vartheta_{m}\left(\vartheta_{m}^{2}-\vartheta_{h}^{2}\right)}\left[\Xi_{m}^{u}, \Xi_{h}\right]+\sum_{\substack{m \in M^{\prime \prime}, h \in M^{\prime} \\
m \neq h}} \frac{\tau \sin \left(\tau_{2} \vartheta_{m}\right)}{\tau_{2}\left(\vartheta_{m}^{2}-\vartheta_{h}^{2}\right)}\left[\Xi_{m}^{u}, \xi_{h}\right] \\
&-\sum_{\substack{m \in M^{\prime \prime}, h \in M^{\prime} \\
m \neq h}} \frac{\tau \vartheta_{h} \sin \left(\tau_{2} \vartheta_{m}\right)}{\tau_{m}\left(\vartheta_{m}^{2}-\vartheta_{h}^{2}\right)}\left[\xi_{m}^{u}, \Xi_{h}\right]+\sum_{\substack{m \in M^{\prime \prime}, h \in M^{\prime} \\
m \neq h}} \frac{\tau_{2}\left(\cos \left(\tau_{2} \vartheta_{m}\right)-1\right)}{\tau_{2}\left(\vartheta_{m}^{2}-\vartheta_{h}^{2}\right)}\left[\xi_{m}^{u}, \xi_{h}\right] \\
&+\left[V, \frac{\tau}{2} X_{1}^{u}+\sum_{m \in \mathcal{M}} \frac{\tau \tau_{2} \vartheta_{m}-\tau \sin \left(\tau_{2} \vartheta_{m}\right)}{\tau_{2}^{2} \vartheta_{m}^{2}} \underset{\substack{u \\
m}}{\sum_{m \in \mathcal{M}}} \frac{\tau\left(1-\cos \left(\tau_{2} \vartheta_{m}\right)\right)}{\tau_{2}^{2} \vartheta_{m}^{2}} \xi_{m}^{u}\right] \\
&\left.-\sum_{m \in \mathcal{M}} \sin \left(\tau_{2} \vartheta_{m}\right) \Xi_{m}^{u}+\sum_{m \in \mathcal{M}}\left(1-\cos \left(\tau_{2} \vartheta_{m}\right)\right) \xi_{m}^{u}\right) . \\
&
\end{aligned}
$$

For $(5)$, we let $\alpha(s)=(\tilde{p}, \cos s v+\sin s \zeta)$, so that $a_{1}(s)=e, a_{2}(s)=$ $\cos s v+\sin s \zeta, \dot{a}_{1}(0)=0$ and $\dot{a}_{2}(0)=\zeta$. Recall that $X_{2}=\sum_{m \in M} \xi_{m}$, where $\xi_{m} \in W_{m}\left(Z_{0}\right)$ and let $\vartheta_{m}=\vartheta_{m}\left(Z_{0}\right)$ and $\Xi_{m}=j\left(Z_{0}\right) \xi_{m} / \vartheta_{m}$. Here $Z_{0}^{s}=$ $\cos s \frac{\tau_{2}}{\tau} Z_{0}+\sin s \zeta$, so $\vartheta_{m}^{s}=\vartheta_{m}\left(Z_{0}^{s}\right)$ and $\vartheta_{m}^{0}=\frac{\tau_{2}}{\tau} \vartheta_{m}$ for $m \in \mathcal{M}$ but $\dot{\vartheta}_{m}^{0}$ cannot, in general, be simplified. Let $\xi_{m}^{s}$ denote the component of $\cos s\left(\frac{1}{\tau} V+\right.$ $\left.\frac{\tau_{2}}{\tau} X_{2}\right)$ in $W_{m}\left(Z_{0}^{s}\right)$. Let $\Xi_{m}^{s}$ denote $j\left(Z_{0}^{s}\right) \xi_{m}^{s} / \vartheta_{m}^{s}$. Note that if $Z_{0} \notin \mathcal{U}$ (see Proposition 2.19), then $\vartheta_{m}^{s}-\vartheta_{h}^{s} \rightarrow 0$ need not imply that $m=h$, and $\vartheta_{m}^{s} \rightarrow 0$ 
need not imply $\vartheta_{m}=0$. Finally, $\xi_{m}^{0}=0$ need not imply $\dot{\xi}_{m}^{0}=0$. Thus, there is very little simplification that can be accomplished. Thus, we may only write the following:

$$
\begin{aligned}
& \left(\mathrm{Id}-\tilde{\Phi}_{\tau *_{\tilde{p}}}\right)(0, \zeta) \\
& =\left(-\tau\left(\dot{X}_{1}^{0}+\sum_{n \in N} \dot{\xi}_{n}^{0}\right)-\sum_{m \in M^{\prime}}\left(\frac{\tau \dot{\vartheta}_{m}^{0}}{\vartheta_{m}^{0}}\right) \xi_{m}^{0}\right. \\
& -\left.\sum_{n \in N} \frac{\tau^{2}}{2} \frac{d}{d s}\right|_{0}\left(J_{s} \xi_{n}^{s}\right)+\sum_{m \in M^{\prime \prime}} \frac{\cos \left(\tau \vartheta_{m}^{0}\right)-1}{\vartheta_{m}^{0}} \dot{\Xi}_{m}^{0}-\sum_{m \in M^{\prime \prime}} \frac{\sin \left(\tau \vartheta_{m}^{0}\right)}{\vartheta_{m}^{0}} \dot{\xi}_{m}^{0} \\
& -\tau \zeta+\sum_{m \in M^{\prime}} \frac{\tau}{\vartheta_{m}^{0}}\left[\dot{X}_{1}^{0}+\sum_{n \in N} \dot{\xi}_{n}^{0}, \Xi_{m}^{0}\right]-\sum_{\substack{m \in M, h \in M^{\prime} \\
\vartheta_{m}^{0}=\vartheta_{h}^{0}}} \frac{\tau}{2 \vartheta_{m}^{0}}\left(\left[\dot{\xi}_{m}^{0}, \Xi_{h}^{0}\right]+\left[\xi_{h}^{0}, \dot{\Xi}_{m}^{0}\right]\right) \\
& -\sum_{m \in M^{\prime}, n \in N} \frac{\tau^{2}}{2 \vartheta_{m}^{0}}\left[\Xi_{m}^{0},\left.\frac{d}{d s}\right|_{0}\left(J_{s} \xi_{n}^{s}\right)\right]+\sum_{m \in M^{\prime}, n \in N} \frac{\tau}{\vartheta_{m}^{0}{ }^{2}}\left[\xi_{m}^{0},\left.\frac{d}{d s}\right|_{0}\left(J_{s} \xi_{n}^{s}\right)\right] \\
& -\sum_{\substack{m, h \in M^{\prime} \\
\vartheta_{m}^{0} \neq \vartheta_{h}^{0}}} \frac{\tau\left(\dot{\vartheta}_{h}^{0} \vartheta_{m}^{0}-\vartheta_{h}^{0} \dot{\vartheta}_{m}^{0}\right)}{\vartheta_{h}^{0}\left(\vartheta_{m}^{0}-\vartheta_{h}^{0}\right)}\left[\Xi_{m}^{0}, \xi_{h}^{0}\right] \\
& -\sum_{\substack{m, h \in M^{\prime} \\
\vartheta_{m}^{0}=\vartheta_{h}^{0}}} \frac{\tau\left(3 \dot{\vartheta}_{h}^{0}+\dot{\vartheta}_{m}^{0}\right)}{4 \vartheta_{m}^{0}{ }^{2}}\left[\Xi_{m}^{0}, \xi_{h}^{0}\right]-\sum_{\substack{m, h \in M^{\prime} \\
\vartheta_{m}^{0}=\vartheta_{h}^{0}}} \frac{\tau^{2}\left(\dot{\vartheta}_{m}^{0}-\dot{\vartheta}_{h}^{0}\right)}{8 \vartheta_{m}^{0}}\left(\left[\Xi_{m}^{0}, \Xi_{h}^{0}\right]+\left[\xi_{m}^{0}, \xi_{h}^{0}\right]\right) \\
& +\sum_{\substack{m \in M^{\prime \prime}, h \in M^{\prime} \\
\vartheta_{m}^{0} \neq \vartheta_{h}^{0}}} \frac{\vartheta_{h}^{0}\left(\cos \left(\tau \vartheta_{m}^{0}\right)-1\right)}{\vartheta_{m}^{0}\left(\vartheta_{m}^{0}-\vartheta_{h}^{0^{2}}\right)}\left[\dot{\Xi}_{m}^{0}, \Xi_{h}^{0}\right]+\sum_{\substack{m \in M^{\prime \prime}, h \in M^{\prime} \\
\vartheta_{m}^{0} \neq \vartheta_{h}^{0}}} \frac{\sin \left(\tau \vartheta_{m}^{0}\right)}{\vartheta_{m}^{0}-\vartheta_{h}^{0}}\left[\dot{\Xi}_{m}^{0}, \xi_{h}^{0}\right] \\
& -\sum_{\substack{m \in M^{\prime \prime}, h \in M^{\prime} \\
\vartheta_{m}^{0} \neq \vartheta_{h}^{0}}} \frac{\vartheta_{h}^{0} \sin \left(\tau \vartheta_{m}^{0}\right)}{\vartheta_{m}^{0}\left(\vartheta_{m}^{0}{ }^{2}-\vartheta_{h}^{0}\right)}\left[\dot{\xi}_{m}^{0}, \Xi_{h}^{0}\right]+\sum_{\substack{m \in M^{\prime \prime}, h \in M^{\prime} \\
\vartheta_{m}^{0} \neq \vartheta_{h}^{0}}} \frac{\cos \left(\tau \vartheta_{m}^{0}\right)-1}{\vartheta_{m}^{0}-\vartheta_{h}^{0^{2}}}\left[\dot{\xi}_{m}^{0}, \xi_{h}^{0}\right] \\
& +\left[V, \frac{\tau}{2}\left(\dot{X}_{1}^{0}+\sum_{n \in N} \dot{\xi}_{n}^{0}\right)-\sum_{m \in M^{\prime}} \frac{2 \dot{\vartheta}_{m}^{0}}{\vartheta_{m}^{0}{ }^{2}} \Xi_{m}^{0}\right. \\
& \left.+\sum_{m \in M^{\prime}} \frac{1}{\vartheta_{m}^{0}} \dot{\Xi}_{m}^{0}+\left.\frac{\tau^{2}}{6} \sum_{n \in N} \frac{d}{d s}\right|_{0}\left(J_{s} \xi_{n}^{s}\right)\right]
\end{aligned}
$$




$$
\begin{aligned}
& +\left[V, \sum_{m \in M^{\prime \prime}} \frac{\tau \vartheta_{m}^{0}-\sin \left(\tau \vartheta_{m}^{0}\right)}{\tau \vartheta_{m}^{0}{ }^{2}} \dot{\Xi}_{m}^{0}+\sum_{m \in M^{\prime \prime}} \frac{1-\cos \left(\tau \vartheta_{m}^{0}\right)}{\left.\tau \vartheta_{m}^{0} \dot{\xi}_{m}^{0}\right]}\right. \\
& -\sum_{m \in M^{\prime}} \tau \dot{\vartheta}_{m}^{0} \Xi_{m}^{0}-\left.\sum_{n \in N} \tau \frac{d}{d s}\right|_{0}\left(J_{s} \xi_{n}^{s}\right)-\sum_{m \in M^{\prime \prime}} \sin \left(\tau \vartheta_{m}^{0}\right) \dot{\Xi}_{m}^{0} \\
& \left.+\sum_{m \in M^{\prime \prime}}\left(1-\cos \left(\tau \vartheta_{m}^{0}\right)\right) \dot{\xi}_{m}^{0}\right) .
\end{aligned}
$$

Case V: DG-Multiplier. As is clear from formula (V-5), a tractable closed form for the DG-multiplier that will work for general two-step nilmanifolds is not plausible. Section 4.2 applies the formulas in Case $\mathrm{V}$ to a specific five-dimensional example.

\section{Special cases and examples}

\subsection{One-dimensional center/Heseinberg manifolds}

We consider the special case where the two-step nilpotent Lie group $G$ has a one-dimensional center; i.e., $G$ is a Heisenberg Lie group. Here we need only Cases I, II and III. To see this, if $V \notin \mathfrak{z}$, then $[V, \mathfrak{g}]=\mathfrak{z}$, and so $Z_{V}^{\perp}=0$ for all $V \neq 0$. With this assumption, Case III becomes tractable, as formula (III-3) never applies.

Let $n=\operatorname{dim}(G)$. Note that $\operatorname{dim} \mathfrak{z}=1$ implies $\operatorname{ker} j(Z)=\{0\}$ for all $Z \in$ $\mathfrak{z}, Z \neq 0$. Let $Z$ be a unit vector in $\mathfrak{z}$.

Case I: Let $\gamma=\exp \left(V^{*}+Z^{*}\right)$, with $V^{*} \neq 0$. Then by Theorem 2.11 $\tau=\left|V^{*}\right|>0$. Recall from (I-DG) that the DG-multiplier is

$$
\frac{1}{\tau^{(\operatorname{dim} C(\gamma, G)-1) / 2}|\operatorname{det} M|},
$$

where $M=\left\langle Z,\left[V, Y^{\perp}\right]\right\rangle$, and $Y^{\perp}$ is a unit normal to $C(\log \gamma, \mathfrak{g})$. One easily computes that $Y^{\perp}=j(Z) V^{*} /\left|j(Z) V^{*}\right|$. To see this, note that $[V, Y]=0$ if and only if $\left\langle Z,\left[V^{*}, Y\right]\right\rangle=0$ if and only if $\left\langle j(Z) V^{*}, Y\right\rangle=0$. This also implies that $\operatorname{dim} C(\gamma, G)=n-1$. Finally, $|\operatorname{det} M|=\left|\left\langle Z,\left[V^{*}, j(Z) V^{*}\right]\right\rangle\right| /\left|j(Z) V^{*}\right|$ $=\left|j(Z) V^{*}\right|$. Thus, the DG-multiplier simplifies to

$$
\frac{1}{\left|V^{*}\right|^{(n-2) / 2}\left|j(Z) V^{*}\right|} .
$$


Case II: Let $\gamma=\exp \left(Z^{*}\right)$, with $\tau=\left|Z^{*}\right|>0$. Recall from (II-DG) that the DG-multiplier in this case is

$$
\tau^{-(n-1) / 2} \prod_{m=1}^{\mu}\left(\frac{2\left(1-\cos \vartheta_{m}\left(Z^{*}\right)\right)}{\vartheta_{m}\left(Z^{*}\right)^{2}}\right)^{-\operatorname{dim} W_{m}\left(Z^{*}\right) / 4} .
$$

Because $\mathfrak{z}$ is one-dimensional, the eigenvalues of $j\left(Z^{*}\right)$ are of the form $\vartheta_{m}\left(Z^{*}\right)=\left|Z^{*}\right| \vartheta_{m}(Z)=\left|Z^{*}\right| t_{m}, m=1, \ldots, \mu$. The multiplier for Case II thus reduces to

$$
\left|Z^{*}\right|^{n-1} 2^{-(n-1) / 4} \prod_{m=1}^{\mu}\left(\frac{1-\cos \left(\left|Z^{*}\right| t_{m}\right)}{t_{m}^{2}}\right)^{-\operatorname{dim} W_{m}(Z) / 4} .
$$

Case III: Let $\gamma=\exp \left(Z^{*}\right)$, with $0<\tau<\left|Z^{*}\right|$.

Recall that $\vartheta_{m}\left(Z^{*}\right)=\left|Z^{*}\right| t_{m}, m=1, \ldots, \mu$ and $Z=Z^{*} /\left|Z^{*}\right|$. By $[12$, 14,26], or Theorem 2.11, the periods of $\gamma$ are of the form

$$
\left\{\sqrt{\frac{4 \pi k_{m}}{t_{m}^{2}}\left(t_{m}\left|Z^{*}\right|-\pi k_{m}\right)}, 1 \leq k_{m}<\frac{t_{m}\left|Z^{*}\right|}{2 \pi}, k_{m} \in \mathbb{Z}\right\} .
$$

Since $\tau$ is a period of $\gamma$, there exists $m_{0} \in\{1, \ldots, \mu\}$ and $k_{m_{0}} \in \mathbb{Z}^{+}$such that $2 \pi k_{m_{0}}<t_{m_{0}}\left|Z^{*}\right|$ and such that $\tau^{2}=2\left(\frac{2 \pi k_{m_{0}}}{t_{m_{0}}}\right)\left|Z^{*}\right|-\left(\frac{2 \pi k_{m_{0}}}{t_{m_{0}}}\right)^{2}$. Let

$$
M_{\tau}=\left\{m \in \mathcal{M}: \exists k_{m} \in \mathbb{Z}^{+}, \frac{k_{m}}{t_{m}}=\frac{\left|Z^{*}\right|-\sqrt{\left|Z^{*}\right|^{2}-\tau^{2}}}{2 \pi}\right\} .
$$

One easily checks that if $m \in M_{\tau}$, then $k_{m_{0}} / t_{m_{0}}=k_{m} / t_{m}$. We set

$$
\mathfrak{v}_{\tau}=\oplus_{m \in M_{\tau}} W_{m}(Z), \quad \mathfrak{v}_{\tau}^{\perp}=\oplus_{m \notin M_{\tau}} W_{m}(Z) .
$$

Let $z_{0}=\sqrt{\frac{\pi k_{m}}{t_{m}\left|Z^{*}\right|-\pi k_{m}}}$, and $x_{2}=\sqrt{1-z_{0}^{2}}$. Then by Definition 2.7

$$
\tilde{F}(\tau, \gamma)=\left\{\left(\tilde{p}, X_{2}+Z_{0}\right): Z_{0}=z_{0} Z, X_{2} \in \mathfrak{v}_{\tau},\left|X_{2}\right|=x_{2}\right\} .
$$

Let $v_{\tilde{p}} \in \tilde{F}(\tau, \gamma)$. Then

$$
T_{v_{\tilde{p}}} \hat{F}(\tau, \gamma)=\operatorname{span}\left\{\left(U^{\prime}, 0\right),\left(0, U^{\prime \prime}\right),(0, v): U^{\prime} \in \mathfrak{g}, U^{\prime \prime} \in \mathfrak{v}_{\tau}, U^{\prime \prime} \perp v\right\} .
$$

To calculate the wave invariant, we need only (1) $\tilde{\Phi}_{\tau *_{v_{\tilde{p}}}}\left(0, v^{\perp}\right)$ where $v^{\perp} \in \operatorname{span}\left\{X_{2}, Z_{0}\right\}, v \perp v^{\perp}$, and (2) $\tilde{\Phi}_{\tau * v_{\tilde{p}}}(0, U), U \in \mathfrak{v}, U \perp \mathfrak{v}_{\tau}$. 
For all $m \notin M_{\tau}$, let $\xi_{m}^{1}$ be a unit vector of $W_{m}(Z)$. Let $\Xi_{m}^{1}=$ $j(Z) \xi_{m}^{1} / \vartheta_{m}(Z)$. One easily checks that $\xi_{m}^{1}$ and $\Xi_{m}^{1}$ are orthogonal unit vectors in $W_{m}(Z)$. Let $\xi_{m}^{2}$ be a unit vector in $W_{m}(Z)$ that is orthogonal to both $\xi_{m}^{1}$ and $\Xi_{m}^{1}$. Proceed until we have $\left\{\xi_{m}^{1}, \Xi_{m}^{1}, \xi_{m}^{2}, \Xi_{m}^{2}, \ldots, \xi_{m}^{a_{m}}, \Xi_{m}^{a_{m}}\right\}$, an orthonormal basis of $W_{m}(Z)$ such that $\Xi_{m}^{\alpha}=j(Z) \xi_{m}^{\alpha} / \vartheta_{m}(Z), \alpha=1, \ldots, a_{m}$. We now have

$$
\left\{\xi_{m}^{\alpha}, \Xi_{m}^{\alpha}: m \notin M_{\tau}, \alpha=1, \ldots, a_{m}\right\}
$$

an (ordered) basis of $\mathfrak{v}_{\tau}^{\perp}$. Let $\left\{E_{1}, \cdots, E_{n}\right\}$ be any (ordered) orthonormal basis of $\mathfrak{g}$, and let $\left\{\bar{X}_{2}, Y_{1}, \ldots, Y_{d}\right\}$ be any (ordered) orthonormal basis of $\mathfrak{v}_{\tau}$, where $\bar{X}_{2}=X_{2} /\left|X_{2}\right|$.

We set

$$
\mathcal{E}=\left\{\left(E_{1}, 0\right), \ldots,\left(E_{n}, 0\right),\left(0, Y_{1}\right), \ldots,\left(0, Y_{d}\right),(0, v)\right\}
$$

Then

$$
\begin{aligned}
\mathcal{F}= & \left\{\left(0, E_{1}\right), \ldots,\left(0, E_{n}\right),\left(-Y_{1},-j\left(Z_{0}\right) Y_{1}\right), \ldots,\left(-Y_{d},-j\left(Z_{0}\right) Y_{d}\right),\right. \\
& \left.\left(-v,-j\left(Z_{0}\right) v\right)\right\}
\end{aligned}
$$

We set

$$
\mathcal{V}=\left\{\left(0, \xi_{m}^{\alpha}\right),\left(0, \Xi_{m}^{\alpha}\right),\left(0, v^{\perp}\right): m=1, \ldots, \mu, m \notin M_{\tau}, \alpha=1, \ldots, a_{m}\right\} .
$$

We now calculate $T \mathcal{V} \wedge \mathcal{F}$ as a multiple of $\mathcal{V} \wedge \mathcal{E}$. Note that since $\mathfrak{z}$ is one-dimensional, $\left[\xi_{m}^{\alpha}, X_{2}\right]=0$ since $X_{2} \in \mathfrak{v}_{\tau}$ and $\xi_{m}^{\alpha} \perp \mathfrak{v}_{\tau}$. By (III-2),

$$
T\left(0, \xi_{m}^{\alpha}\right)=\left(-s_{m} \xi_{m}^{\alpha}-c_{m} \Xi_{m}^{\alpha},\left(1-\cos \left(\tau \vartheta_{m}\right)\right) \xi_{m}^{\alpha}-\sin \left(\tau \vartheta_{m}\right) \Xi_{m}^{\alpha}\right)
$$

and

$$
T\left(0, \Xi_{m}^{\alpha}\right)=\left(c_{m} \xi_{m}^{\alpha}-s_{m} \Xi_{m}^{\alpha}, \sin \left(\tau \vartheta_{m}\right) \xi_{m}^{\alpha}+\left(1-\cos \left(\tau \vartheta_{m}\right)\right) \Xi_{m}^{\alpha}\right)
$$

Here $c_{m}=\left(1-\cos \left(\tau \vartheta_{m}\right)\right) / \vartheta_{m}$ and $s_{m}=\sin \left(\tau \vartheta_{m}\right) / \vartheta_{m}$, and recall that $\vartheta_{m}=$ $t_{m}\left|Z_{0}\right|$. Since $T\left(0, \xi_{m}^{\alpha}\right) \wedge T\left(0, \Xi_{m}^{\alpha}\right)$ is wedged with $\left(0, E_{1}\right) \wedge \cdots \wedge\left(0, E_{n}\right)$, the contribution of $T\left(0, \xi_{m}^{\alpha}\right) \wedge T\left(0, \Xi_{m}^{\alpha}\right)$ to $T \mathcal{V} \wedge \mathcal{F}$ is $\left(s_{m}^{2}+c_{m}^{2}\right)\left(\xi_{m}^{\alpha}, 0\right) \wedge\left(\Xi_{m}^{\alpha}, 0\right)$. Note that $\left(s_{m}^{2}+c_{m}^{2}\right)=2\left(1-\cos \left(\tau \vartheta_{m}\right)\right) / \vartheta_{m}^{2}$.

Now $\tau=\sqrt{4 \pi k_{m_{0}} / t_{m_{0}}^{2}} \sqrt{t_{m_{0}}|Z|-\pi k_{m_{0}}}$, and after plugging this into the value for $\left|Z_{0}\right|$ from above, we see that $\tau \vartheta_{m}=\tau t_{m}\left|Z_{0}\right|=2 \pi k_{m_{0}} t_{m} / t_{m_{0}}$. Now, by the definition of $M_{\tau}$, for $m \notin M_{\tau}, \tau \vartheta_{m} \notin 2 \pi \mathbb{Z}^{+}$. Thus, for $m \notin M_{\tau},(1-$ $\left.\cos \left(\tau \vartheta_{m}\right)\right) \neq 0$. Finally, by (III-1), $T\left(0, v^{\perp}\right)=\left(\tau \frac{\left|X_{2}\right|^{2}}{\left|Z_{0}\right|^{2}} v^{\perp},-\tau \frac{\left|X_{2}\right|}{\left|Z_{0}\right|} j\left(Z_{0}\right) X_{2}\right)$. 
Putting this altogether, we see that the DG-multiplier for Case III is

$$
\tau^{-1 / 2}\left|Z_{0}\right|\left|X_{2}\right|^{-1} \prod_{m \notin M_{\tau}}\left(\frac{2\left(1-\cos \left(\tau t_{m}\left|Z_{0}\right|\right)\right)}{t_{m}^{2}\left|Z_{0}\right|^{2}}\right)^{-\operatorname{dim} W_{m}(Z) / 4}
$$

where $\left|Z_{0}\right|=\frac{1}{\tau}\left(\left|Z^{*}\right|-\sqrt{\left|Z^{*}\right|^{2}-\tau^{2}}\right)$ and $\left|X_{2}\right|=\sqrt{1-\left|Z_{0}\right|^{2}}$.

Remark 4.1. (Example 2.15, continued) Note that in the case of a onedimensional center, the DG-multiplier in Case II and III depends only on the length $\tau$, the value $\left|Z^{*}\right|$ and on the eigenvalues of $j(Z)$ and the dimensions of the invariant subspaces of $j(Z)$, for $Z$ a unit vector in $\mathfrak{z}$. In Example 2.15, we argued that the wave invariants, not just the DG-multiplier, associated to the central periods were equal for the Heisenberg manifolds considered there. We provide details of this argument now. Note that the only other consideration in the calculation of the wave trace is $\operatorname{Vol}(\Gamma \backslash \tilde{F}(\tau, \gamma))$ and $\operatorname{dim} \tilde{F}(\tau, \gamma)$. We already showed that $\operatorname{dim} \tilde{F}(\tau, \gamma)$ is equal for the pairs of examples considered in Example 2.15. The central periods correspond to Cases II and III. In Case II, $\Gamma \backslash \tilde{F}(\tau, \gamma)=\Gamma \backslash H_{n} \times\{Z\}$. The volume of this will be equal in the pairs of examples, because isospectral manifolds must have the same volume. For Case III, $\Gamma \backslash \tilde{F}(\tau, \gamma)=\Gamma \backslash H_{n} \times S_{x_{2}}\left(\mathfrak{v}_{\tau}\right)$, where $S_{x_{2}}\left(\mathfrak{v}_{\tau}\right)$ is the sphere of radius $x_{2}=\left|X_{2}\right|$ in $\mathfrak{v}_{\tau}$. Again, the volume in this case is equal for the pairs of examples, since $\mathfrak{v}_{\tau}, x_{2}$ and $z_{0}=\left|Z_{0}\right|$ depend only on $\tau,\left|Z^{*}\right|$, and the eigenvalues and dimensions of invariant subspaces of $j(Z)$ for $Z$ a unit vector in $\mathfrak{z}$. By Theorem 2.16, these values will be the same for isospectral Heisenberg manifolds. (The wave invariants for Case I are compared in Example 2.15.)

\subsection{Five-dimensional example of the Poincaré map}

We now include a nontrivial example in order to demonstrate explicitly why we need all five cases to compute the Poincaré map for general two step nilmanifolds.

Let $\mathfrak{g}=\operatorname{span}\left\{X, Y_{1}, Y_{2}, Z_{1}, Z_{2}\right\}$ where $\left[X, Y_{i}\right]=Z_{i}, i=1,2$ and all other basis brackets (not determined by skew-symmetry) are zero. Let $G$ be the simply connected Lie group with Lie algebra $\mathfrak{g}$. We use the inner product on $\mathfrak{g}$ for which the given basis is orthonormal. With this metric, letting $Z=z_{1} Z_{1}+z_{2} Z_{2}$,

$$
j(Z)=\left(\begin{array}{ccc}
0 & -z_{1} & -z_{2} \\
z_{1} & 0 & 0 \\
z_{2} & 0 & 0
\end{array}\right)
$$


and the eigenvalue curves of $j(Z)$ are $\Theta_{0}(Z)=0$ and $\Theta(Z)=|Z|$. The invariant subspaces associated to these eigenvalue curves are $W_{0}(Z)=$ $\operatorname{span}\left\{-z_{2} Y_{1}+z_{1} Y_{2}\right\}$ and $W(Z)=\operatorname{span}\left\{X, z_{1} Y_{1}+z_{2} Y_{2}\right\}$. Note that $\mathfrak{v}=$ $\operatorname{span}\left\{X, Y_{1}, Y_{2}\right\}$ and $\mathfrak{z}=\operatorname{span}\left\{Z_{1}, Z_{2}\right\}$.

Let $\gamma=\exp (V+Z)$ where $V \in \mathfrak{v}$ and $Z \in \mathfrak{z}$. We analyze the periods of $\gamma$ and the DG-multiplier associated to each of these periods.

We consider the five cases $\mathrm{I}-\mathrm{V}$ as listed above, as they can all occur. Case $I . V \neq 0$ and $Z_{V}^{\perp}=0$ and $\tau=|V|$.

Write $V=x X+y_{1} Y_{1}+y_{2} Y_{2}$. If $x \neq 0$, then $C(V, \mathfrak{g})=\operatorname{span}\left\{V, Z_{1}, Z_{2}\right\}$ so that $C(V, \mathfrak{g})^{\perp}=\operatorname{span}\left\{Y_{1}^{\perp}, Y_{2}^{\perp}\right\}$, where $Y_{i}^{\perp}=\left(-y_{i} X+x Y_{i}\right) / \sqrt{x^{2}+y_{i}^{2}}$, $i=1,2$. As $C(V, \mathfrak{g})^{\perp}$ is two-dimensional, we must calculate the $2 \mathrm{x} 2$ matrix $M=\left\langle Z_{i},\left[V, Y_{j}^{\perp}\right]\right\rangle$ where $i, j=1,2$. Thus

$$
M=\left(\begin{array}{cc}
\sqrt{x^{2}+y_{1}^{2}} & \frac{y_{1} y_{2}}{\sqrt{x^{2}+y_{1}^{2}}} \\
\frac{y_{1} y_{2}}{\sqrt{x^{2}+y_{2}^{2}}} & \sqrt{x^{2}+y_{2}^{2}}
\end{array}\right)
$$

We conclude that if $x \neq 0$, then by (I-DG) the DG-multiplier for Case I is

$$
\frac{1}{|V||\operatorname{det} M|} \text {. }
$$

If $x=0$, then $C(V, \mathfrak{g})=\operatorname{span}\left\{Y_{1}, Y_{2}, Z_{1}, Z_{2}\right\}$ so that $C(V, \mathfrak{g})^{\perp}=$ $\operatorname{span}\{X\}$. In this case, $M$ is a $1 \times 1$ matrix. Note that $[V, \mathfrak{g}]=$ $\operatorname{span}\left\{y_{1} Z_{1}+y_{2} Z_{2}\right\}$. Thus $|\operatorname{det} M|=\left|\left\langle\frac{1}{|V|}\left(y_{1} Z_{1}+y_{2} Z_{2}\right),[V, X]\right\rangle\right|=|V|$. We conclude that if $x=0$, then by (I-DG) the DG-multiplier for Case I is

$$
\frac{1}{|V|^{5 / 2}} \text {. }
$$

Case II. $V=0$ and $\tau=|Z|$.

Note that $\operatorname{dim} W(Z)=2$, so that by (II-DG), the DG-multiplier for Case II is

$$
\frac{1}{|Z| 2^{1 / 2}(1-\cos |Z|)^{1 / 2}} .
$$

By the Clean Intersection Hypothesis, $\cos |Z| \neq 1$.

Case III. $V=0$ and $\tau<|Z|$.

We write $Z=\Upsilon\left(z_{1} Z_{1}+z_{2} Z_{2}\right)$, with $z_{1}^{2}+z_{2}^{2}=1$ and $\Upsilon>0$. We also denote $\bar{Z}=z_{1} Z_{1}+z_{2} Z_{2}$, the unit vector in the direction $Z$. We denote $Y_{Z}=z_{1} Y_{1}+z_{2} Y_{2}$ and $Y_{Z^{\perp}}=-z_{2} Y_{1}+z_{1} Y_{2}$ and $Z^{\perp}=-z_{2} Z_{1}+z_{1} Z_{2}$. Now 
the Lie group $G$ with the given metric is an example of a two-step nilpotent Lie group that is Heisenberg-like [26]. In particular, a two-step nilpotent Lie group is Heisenberg-like if and only if all eigenvalue curves are of the form $\theta_{m}(Z)=c_{m}|Z|$, for some constant $c_{m}$. Here, the eigenvalue curves are $\Theta_{0}(Z)=0$ and $\Theta(Z)=|Z|$. In the case of Heisenberg-like metric Lie groups, the periods of elements take on a nice form [26]. In particular, the periods of $\exp (Z)$ are

$$
\left\{|Z|, \sqrt{4 \pi k(|Z|-\pi k)}, 1 \leq k<\frac{|Z|}{2 \pi}, k \in \mathbb{Z}\right\} .
$$

One checks that

$$
\begin{gathered}
\tilde{F}(\tau, \gamma)=\left\{(\tilde{p}, v): p \in G, v=X_{2}+Z_{0}, Z_{0}=z_{0} \bar{Z}\right. \\
\left.X_{2}=x_{2} U, U \in W(Z),|U|=1\right\}
\end{gathered}
$$

where $x_{2}=\sqrt{(|Z|-2 \pi k) /(|Z|-\pi k)}$ and $z_{0}=\sqrt{\pi k /(|Z|-\pi k)}$. Note that $\operatorname{ker} j(Z)=\operatorname{span}\left\{Y_{Z^{\perp}}\right\}$ and $W(Z)=\operatorname{span}\left\{X, Y_{Z}\right\}$. So that $U=x X+y Y_{Z}$, with $x^{2}+y^{2}=1$. Denote $U^{\perp}=-y X+x Y_{Z}=j(\bar{Z}) U$. Let $v_{\tilde{p}} \in \tilde{F}(\tau, \gamma)$, then

$$
T_{v_{\tilde{p}}} \tilde{F}(\tau, \gamma)=\operatorname{span}\left\{\left(U^{\prime}, U^{\prime \prime}\right): U^{\prime} \in \mathfrak{g}, U^{\prime \prime} \in W(Z), U^{\prime \prime} \perp v\right\}
$$

A basis of $\mathcal{E}$ is

$$
\mathcal{E}=\left\{(X, 0),\left(Y_{Z}, 0\right),\left(Y_{Z^{\perp}}, 0\right),(\bar{Z}, 0),\left(Z^{\perp}, 0\right),(0, v),\left(0, U^{\perp}\right)\right\}
$$

Then

$$
\mathcal{F}=\left\{(0, X),\left(0, Y_{Z}\right),\left(0, Y_{Z^{\perp}}\right),(0, \bar{Z}),\left(0, Z^{\perp}\right),\left(-v,-j\left(Z_{0}\right) v\right),\left(-U^{\perp}, z_{0} U\right)\right\}
$$

A basis of $\mathcal{V}$ is

$$
\mathcal{V}=\left\{\left(0, v^{\perp}\right),\left(0, Y_{Z^{\perp}}\right),\left(0, Z^{\perp}\right)\right\}
$$

where $v^{\perp}=-\frac{z_{0}}{x_{2}} X_{2}+\frac{x_{2}}{z_{0}} Z_{0}$.

We wish to calculate $T \mathcal{V} \wedge \mathcal{F}$ as a multiple of $\mathcal{V} \wedge \mathcal{E}$.

From (III-1), we conclude that $T\left(0, v^{\perp}\right)=\left(\frac{\tau x_{2}^{2}}{z_{0}^{2}} v^{\perp}, \star\right)$. From (III-2), we conclude that $T\left(0, Y_{Z^{\perp}}\right)=\left(-\tau Y_{Z^{\perp}}+\frac{\tau x_{2} y}{z_{0}} Z^{\perp}, \star\right)$. Here the $\star s$ refer to values that we do not need to calculate, because we will be wedging with values that will eliminate the contribution of $\star$ to $T \mathcal{V} \wedge \mathcal{F}$. 
While $\tilde{\Phi}_{\tau *_{v \tilde{p}}}\left(0, Z^{\perp}\right)$ does not depend on the curve $a_{2}(s)$, the expression for it given in (III-3) does, and its evaluation will involve more details. We continue the notation of Section 2 and Section 3 Case III(3). Recall that $a_{2}(s)=\cos s\left(x_{2} U+z_{0} \bar{Z}\right)+\sin s Z^{\perp}$, so that $Z_{0}^{s}=\cos s z_{0} \bar{Z}+\sin s Z^{\perp}$. Also, $\Theta^{s}=\left|Z_{0}^{s}\right|=\sqrt{\cos ^{2} s z_{0}^{2}+\sin ^{2} s}$. Note that $\left|Z_{0}^{s}\right|$ does not go to zero as $s$ goes to zero, so the set $N=\emptyset$. Using the subspaces $\operatorname{ker} j\left(Z_{0}^{s}\right)=$ $\operatorname{span}\left\{-\sin s Y_{Z}+z_{0} \cos s Y_{Z^{\perp}}\right\}$ and $W\left(Z_{0}^{s}\right)=\operatorname{span}\left\{X, z_{0} \cos s Y_{Z}+\sin s Y_{Z^{\perp}}\right\}$, one computes that, with $X_{0}^{s}=x_{2} \cos s U$,

$$
X_{1}^{s}=\frac{-x_{2} y \cos s \sin s}{\left(\Theta^{s}\right)^{2}}\left(-\sin s Y_{Z}+z_{0} \cos s Y_{Z^{\perp}}\right)
$$

and

$$
\xi^{s}=x x_{2} \cos s X+\frac{x_{2} y z_{0} \cos ^{2} s}{\left(\Theta^{s}\right)^{2}}\left(z_{0} \cos s Y_{Z}+\sin s Y_{Z^{\perp}}\right) .
$$

One easily checks that $\dot{\Theta^{0}}=0, X_{1}^{0}=0, \xi^{0}=x_{2} U, \dot{\xi}^{0}=\frac{y x_{2}}{z_{0}} Y_{Z^{\perp}}$ and $\dot{X}_{1}^{0}=$ $-\dot{\xi}^{0}$. Note that since $\xi^{0} \neq 0$, we have $M^{\prime \prime}=\emptyset$. Using $\Xi^{s}=j\left(Z_{0}^{s}\right) \xi^{s} / \Theta^{s}$, we have $\Xi^{0}=x_{2} U^{\perp}$, and $\dot{\Xi}^{0}=\frac{x x_{2}}{z_{0}} Y_{Z^{\perp}}$. Applying (III-3) with these values, we conclude that

$$
T\left(0, Z^{\perp}\right)=\left(\frac{\tau y x_{2}}{z_{0}} Y_{Z^{\perp}}-\frac{\tau}{2 z_{0}^{2}}\left(1+2 y^{2} x_{2}^{2}+z_{0}^{2}\right) Z^{\perp}, \star\right) .
$$

Now

$$
\begin{aligned}
T \mathcal{V} \wedge \mathcal{F} & =T\left(0, v^{\perp}\right) \wedge T\left(0, Y_{Z^{\perp}}\right) \wedge T\left(0, Z^{\perp}\right) \wedge(0, X) \wedge\left(0, Y_{Z}\right) \wedge\left(0, Y_{Z^{\perp}}\right) \\
& \wedge(0, \bar{Z}) \wedge\left(0, Z^{\perp}\right) \wedge\left(-v,-j\left(Z_{0}\right) v\right) \wedge\left(-U^{\perp}, z_{0} U\right) \\
& = \pm\left(\frac{\tau x_{2}^{2}}{z_{0}^{2}} v^{\perp}, 0\right) \wedge\left(-\tau Y_{Z^{\perp}}+\frac{\tau y x_{2}}{z_{0}} Z^{\perp}, 0\right) \\
& \wedge\left(\frac{\tau x_{2} y}{z_{0}} Y_{Z^{\perp}}-\frac{\tau}{2 z_{0}^{2}}\left(1+2 y^{2} x_{2}^{2}+z_{0}^{2}\right) Z^{\perp}, 0\right) \\
& \wedge(v, 0) \wedge\left(U^{\perp}, 0\right) \wedge(0, X) \wedge \cdots \wedge\left(0, Z^{\perp}\right) \\
& = \pm \frac{\tau^{3}\left(1-z_{0}^{4}\right)}{2 z_{0}^{2}} \mathcal{V} \wedge \mathcal{E} .
\end{aligned}
$$


We use here the fact that both $\left\{X, Y_{Z}, Y_{Z^{\perp}}, \bar{Z}, Z^{\perp}\right\}$ and $\left\{v, v^{\perp}, U^{\perp}, Y_{Z^{\perp}}, Z^{\perp}\right\}$ are orthonormal bases of $\mathfrak{g}$.

The DG-multiplier for Case III is then

$$
\frac{\sqrt{2} z_{0}}{\tau^{3 / 2} \sqrt{1-z_{0}^{4}}}
$$

Recall that $2 \pi \leq 2 \pi k<|Z|$, and $z_{0}^{2}=(\pi k) /(|Z|-\pi k)<1$.

Case $I V . V \neq 0, Z_{V}^{\perp} \neq 0, \tau=\left|V+Z_{V}^{\perp}\right|$.

For this case to occur, we must have $V=\lambda\left(y_{1} Y_{1}+y_{2} Y_{2}\right)$ and $Z_{V}^{\perp}=$ $z\left(-y_{2} Z_{1}+y_{1} z_{2}\right)$, where we assume $y_{1}^{2}+y_{2}^{2}=1$ and $\lambda>0$. We let $Y=$ $y_{1} Y_{1}+y_{2} Y_{2}$ and $Y^{\perp}=-y_{2} Y_{1}+y_{1} Y_{2}$. Define $Z_{Y}=y_{1} Z_{1}+y_{2} Z_{2}$ and $Z_{Y^{\perp}}=$ $-y_{2} Z_{1}+y_{1} Z_{2}$, so $V=\lambda Y$ and $Z_{V}^{\perp}=z Z_{Y^{\perp}}$. Note that $[V, \mathfrak{g}]=\operatorname{span}\left\{Z_{Y}\right\}$. Thus $\operatorname{ker} j\left(Z_{V}^{\perp}\right)=\operatorname{span}_{\mathbb{R}}\{V\}=\operatorname{span}\{Y\}, \quad \log C(\gamma, G)=\operatorname{span}_{\mathbb{R}}\left\{Y, Y^{\perp}, Z_{Y}\right.$, $\left.Z_{Y \perp}\right\}$ and $\log C(\gamma, G)^{\perp}=\mathbb{R} X$. In this case

$$
\tilde{F}(\tau, \gamma)=\left\{(\tilde{p}, v): \tilde{p} \in C(\gamma, G) \tilde{q}, v=\left(V+Z_{V}^{\perp}\right) / \tau\right\}
$$

We let

$$
\mathcal{E}=\left\{(Y, 0),\left(Y^{\perp}, 0\right),\left(Z_{Y}, 0\right),\left(Z_{Y^{\perp}}, 0\right),(0, v)\right\}
$$

Thus

$$
\mathcal{F}=\left\{(0, Y),\left(0, Y^{\perp}\right),\left(0, Z_{Y}\right),\left(0, Z_{Y^{\perp}}\right),(-v, 0)\right\}
$$

and we let

$$
\mathcal{V}=\left\{(X, 0),(0, X),\left(0, Y^{\perp}\right),\left(0, v^{\perp}\right),\left(0, Z_{Y}\right)\right\}
$$

We apply the above values to (IV-DG) in order to compute the DG-multiplier here.

The matrix $M$ is $1 \times 1$, so $\operatorname{det} M=\left\langle Z_{Y},[V, X]\right\rangle=-\lambda$. Because the metric Lie group is Heisenberg-like, we are in the case where $N=\emptyset$. To calculate the matrix $\hat{M}$, we must evaluate the curve $a_{2}(s)$ more closely. In the notation of Case IV(4) and (IV-DG), the curve $a_{2}(s)=\frac{\cos s}{\tau}\left(V+Z_{V}^{\perp}\right)+\sin s Z_{Y}$ produces $Z_{0}^{s}=\frac{z \cos s}{\tau} Z_{Y^{\perp}}+\sin s Z_{Y}$, so that $\Theta^{s}=\sqrt{\sin ^{2} s+z^{2} \cos ^{2} s / \tau^{2}}$. Using the subspaces $\operatorname{ker} j\left(Z_{0}^{s}\right)=\operatorname{span}\left\{-\sin s Y^{\perp}+\cos s z_{0} Y\right\}$ and $W\left(Z_{0}^{s}\right)=$ $\operatorname{span}\left\{X, z_{0} \cos s Y^{\perp}+\sin s Y\right\}$, where $z_{0}=z / \tau$, we compute

$$
\xi^{s}=\frac{\lambda \cos s \sin s}{\tau\left(\Theta^{s}\right)^{2}}\left(\sin s Y+\frac{z \cos s}{\tau} Y^{\perp}\right) .
$$

Thus $\zeta_{\beta}=Z_{Y}, Y_{\delta}^{\perp}=X, U_{\beta}=\dot{\xi}^{0}=\frac{\lambda}{z} Y^{\perp}, J_{\beta}=0, \xi^{\alpha}=X$ and $\Xi^{\alpha}=Y^{\perp}$. Plugging this into $(\mathrm{IV}-\hat{M})$ we obtain the $1 \times 1$ matrix $\hat{M}$ to be $\pm \lambda= \pm|V|$. 
We thus obtain a DG-multiplier of

$$
\tau^{-3 / 2}|V|^{-1}\left|Z_{V}^{\perp}\right| 2^{-1 / 2}\left(1-\cos \left|Z_{V}^{\perp}\right|\right)^{-1 / 2}
$$

By the Clean Intersection Hypothesis, $\left|Z_{V}^{\perp}\right|=\vartheta_{2}\left(Z_{V}^{\perp}\right) \notin 2 \pi \mathbb{Z}$.

Case $V . V \neq 0 Z_{V}^{\perp} \neq 0 \tau<\left|V+Z_{V}^{\perp}\right|$.

As $Z_{V}^{\perp} \neq 0$ we must have $V=\lambda Y$ and $Z_{V}^{\perp}=\Upsilon Z_{Y^{\perp}}$, where $Y=y_{1} Y_{1}+$ $y_{2} Y_{2}, \quad \lambda>0, Y^{\perp}=-y_{2} Y_{1}+y_{1} Y_{2}, Z_{Y^{\perp}}=-y_{2} Z_{1}+y_{1} Z_{2}$ and $y_{1}^{2}+y_{2}^{2}=1$. Note that $[V, \mathfrak{g}]=\mathbb{R} Z_{Y}$ where $Z_{Y}=y_{1} Z_{1}+y_{2} Z_{2}$. As $\tau<\left|V+Z_{V}^{\perp}\right|$, we must have $\tau=\sqrt{|V|^{2}+\tau_{2}^{2}}$ where $\tau_{2}=\sqrt{4 \pi k\left(\left|Z_{V}^{\perp}\right|-\pi k\right)}, k \in \mathbb{Z}^{+}$and $1 \leq$ $k<\left|Z_{V}^{\perp}\right| / 2 \pi$. The fact that $\tau$ must be of this form follows from the fact that $G$ is Heisenberg-like and the expression of the length spectrum given in Case V, above. See [26] for more details.

Now

$$
\begin{aligned}
\tilde{F}(\tau, \gamma)= & \left\{(\tilde{p}, v): \tilde{p} \in C(\gamma, G) \tilde{q}, v=\frac{1}{\tau} V+\frac{\tau_{2}}{\tau}\left(X_{2}+Z_{0}\right),\right. \\
& \left.X_{2}=x_{2} U, Z_{0}=z_{0} \epsilon Z_{Y^{\perp}}, U \in W\left(Z_{Y^{\perp}}\right),|U|=1\right\},
\end{aligned}
$$

where $z_{0}=\sqrt{\pi k /\left(\left|Z_{V}^{\perp}\right|-\pi k\right)}, \quad x_{2}=\sqrt{\left(\left|Z_{V}^{\perp}\right|-2 \pi k\right) /\left(\left|Z_{V}^{\perp}\right|-\pi k\right)}$ and $\epsilon=$ \pm 1 , depending on the sign of $\Upsilon$. That is, $\epsilon Z_{Y^{\perp}}=Z_{V}^{\perp} /\left|Z_{V}^{\perp}\right|$. Note that $x_{2}^{2}+z_{0}^{2}=1$.

Let $v_{\tilde{p}} \in \tilde{F}(\tau, \gamma)$, so $v=\frac{\lambda}{\tau} Y+\frac{\tau_{2}}{\tau}\left(X_{2}+Z_{0}\right), X_{2}=x_{2} U, Z_{0}=z_{0} \epsilon Z_{Y^{\perp}}$ and $U=x X+y Y^{\perp}$, where $x^{2}+y^{2}=1$.

Then

$$
T_{v_{\tilde{p}}} \tilde{F}(\tau, \gamma)=\left\{\left(U^{\prime}, U^{\prime \prime}\right): U^{\prime} \in \log C(\gamma, G), U^{\prime \prime} \in W\left(Z_{Y^{\perp}}\right), v \perp U^{\prime \prime}\right\}
$$

We have

$$
\mathcal{E}=\left\{(Y, 0),\left(Y^{\perp}, 0\right),\left(Z_{Y}, 0\right),\left(Z_{Y^{\perp}}, 0\right),(0, v),\left(0, U^{\perp}\right)\right\}
$$

where $U^{\perp}=j\left(Z_{Y^{\perp}}\right) U=-y X+x Y^{\perp}$. Using the symplectic form in Theorem 2.20, we have

$$
\begin{aligned}
\mathcal{F}= & \left\{(0, Y),\left(0, Y^{\perp}\right),\left(0, Z_{Y}\right),\left(0, Z_{Y^{\perp}}\right),\left(-v,-\frac{\tau_{2}^{2} x_{2} z_{0} \epsilon}{\tau^{2}} U^{\perp}\right),\right. \\
& \left.\left(-U^{\perp}, \frac{\tau_{2} z_{0} \epsilon}{\tau} U\right)\right\}
\end{aligned}
$$


and we choose

$$
\mathcal{V}=\left\{(X, 0),\left(0, v_{1}^{\perp}\right),\left(0, v_{2}^{\perp}\right),\left(0, Z_{Y}\right)\right\}
$$

where

$$
v_{1}^{\perp}=-z_{0} U+\epsilon x_{2} Z_{Y^{\perp}} \quad \text { and } \quad v_{2}^{\perp}=-\frac{\tau_{2}}{\tau} Y+\frac{\lambda}{\tau}\left(x_{2} U+z_{0} \epsilon Z_{Y^{\perp}}\right) .
$$

By $(\mathrm{V}-1)$,

$$
T(X, 0)=([V, X], 0)=\left(-\lambda Z_{Y}, 0\right) .
$$

Note that $[V, \mathfrak{g}]=\mathbb{R} Z_{Y}$, so we may ignore any $Z_{Y}$ and $[V, \mathfrak{g}]$ components in the left hand entry of our remaining calculations.

Because $\mathfrak{g}$ is Heisenberg-like, or by direction computation, $\left[\xi, j\left(Z_{0}\right)^{-1} \xi\right]$ $=\frac{-x_{2}^{2}}{z_{0}^{2}} Z_{0}$. Also, $j\left(Z_{0}\right) X_{2}=x_{2} z_{0} \epsilon U^{\perp}$. Using $(V-2)$, we see that

$$
\begin{aligned}
T\left(0, v_{1}^{\perp}\right) & =\left(-\tau \frac{x_{2}}{z_{0}}\left(X_{2}+Z_{0}\right)+\tau \frac{x_{2}}{z_{0}^{3}} Z_{0}+[V, \star],-\tau_{2} x_{2}^{2} \epsilon U^{\perp}\right) \\
& =\left(\tau \frac{x_{2}^{2}}{z_{0}^{2}} v_{1}^{\perp}+\star,-\tau_{2} x_{2}^{2} \epsilon U^{\perp}\right) .
\end{aligned}
$$

Using (V-3), we see that

$$
T\left(0, v_{2}^{\perp}\right)=\left(-\tau v_{2}^{\perp}+[V, \star],-\frac{\tau_{2} \lambda}{\tau} x_{2} z_{0} \epsilon U^{\perp}\right) .
$$

Finally, we use $(\mathrm{V}-5)$ to evaluate $T\left(0, Z_{Y}\right)$.

Let $a_{2}(s)=\cos s v+\sin s Z_{Y}$. Then $Z_{0}^{s}=\alpha^{s} Z_{Y}+\beta^{s} Z_{Y^{\perp}}$, where $\alpha_{s}=$ $\sin s, \beta_{s}=\cos s \tau_{2} z_{0} \epsilon / \tau$. So $\Theta^{s}=\Theta\left(Z_{0}^{s}\right)=\left|Z_{0}^{s}\right|$. Note that $\dot{Z}_{0}^{0}=Z_{Y}, \Theta^{0}=$ $\tau_{2} z_{0} \epsilon / \tau$ and $\dot{\Theta}^{0}=0$. We conclude $N=\emptyset$ when applying (V-5).

Now $a_{2}(s)=X_{1}^{s}+\xi^{s}+Z_{0}^{s}$, where $X_{1}^{s} \in \operatorname{ker} j\left(Z_{0}^{s}\right)$ and $\xi^{s} \in W\left(Z_{0}^{s}\right)$ for all $s$. We have $\operatorname{ker} j\left(Z_{0}^{s}\right)=\operatorname{span}\left\{\beta_{s} Y-\alpha_{s} Y^{\perp}\right\}$ and $W\left(Z_{0}^{s}\right)=\operatorname{span}\left\{X, \alpha_{s} Y+\right.$ $\left.\beta_{s} Y^{\perp}\right\}$. One easily calculates

$$
X_{1}^{s}=\frac{\cos s}{\tau\left(\Theta^{s}\right)^{2}}\left(\beta_{s} \lambda-\alpha_{s} \tau_{2} x_{2} y\right)\left(\beta_{s} Y-\alpha_{s} Y^{\perp}\right)
$$

and

$$
\xi^{s}=\frac{x x_{2} \tau_{2} \cos s}{\tau} X+\frac{\cos s}{\tau\left(\Theta^{s}\right)^{2}}\left(\alpha_{s} \lambda+\beta_{s} \tau_{2} x_{2} y\right)\left(\alpha_{s} Y+\beta_{s} Y^{\perp}\right)
$$

Note that since $\xi^{0} \neq 0, \quad M^{\prime \prime}=\emptyset . \quad$ Thus $X_{1}^{0}=\frac{\lambda}{\tau} Y, \quad \xi^{0}=\frac{x_{2} \tau_{2}}{\tau} U, \quad \dot{\xi}^{0}=$ $\epsilon\left(\frac{y x_{2}}{z_{0}} Y+\frac{\lambda}{\tau_{2} z_{0}} Y^{\perp}\right)$ and $\dot{X}_{1}^{0}=-\dot{\xi}_{0} . \quad$ As $\quad \Xi^{s}=j\left(Z_{0}^{s}\right) \xi^{s} / \Theta^{s}, \quad \Xi^{0}=\frac{\tau_{2} x_{2} \epsilon}{\tau} U^{\perp}$ 
and $\dot{\Xi}^{0}=\left(\frac{-\lambda}{\tau_{2} z_{0}}\right) X+\left(\frac{x x_{2}}{z_{0}}\right) Y$. Applying (V-5) we compute

$$
\begin{aligned}
T\left(0, Z_{Y}\right) & =\left(\frac{\tau \epsilon}{z_{0}}\left(y x_{2} Y+\frac{\lambda}{\tau_{2}} Y^{\perp}\right)-\frac{2 y x_{2} \lambda \tau}{\tau_{2} z_{0}^{2}} Z_{Y^{\perp}}+\star Z_{Y}, 0\right) \\
& =\left(\frac{\tau \lambda y \epsilon\left(z_{0}^{2}-2\right)}{\tau_{2} z_{0}^{2}} v_{1}^{\perp}-\frac{\tau^{2} y \epsilon x_{2}}{\tau_{2} z_{0}} v_{2}^{\perp}+\frac{x \lambda \tau \epsilon}{\tau_{2} z_{0}} U^{\perp}, 0\right) .
\end{aligned}
$$

Recall that we seek to calculate $T \mathcal{V} \wedge \mathcal{F}=$

$$
\begin{aligned}
T(X, 0) & \wedge T\left(0, v_{1}^{\perp}\right) \wedge T\left(0, v_{2}^{\perp}\right) \wedge T\left(0, Z_{Y}\right) \\
& \wedge(0, Y) \wedge\left(0, Y^{\perp}\right) \wedge\left(0, Z_{Y}\right) \wedge\left(0, Z_{Y^{\perp}}\right) \\
& \wedge\left(-v,-\frac{\tau_{2}^{2} x_{2} z_{0}}{\tau^{2}} U^{\perp}\right) \wedge\left(-U^{\perp}, \frac{\tau_{2} z_{0}}{\tau} U\right)
\end{aligned}
$$

as a multiple of $\mathcal{V} \wedge \mathcal{E}$. First note that $\left\{X, Y, Y^{\perp}, Z_{Y}, Z_{Y^{\perp}}\right\}$ and $\left\{v, v_{1}^{\perp}, v_{2}^{\perp}\right.$, $\left.U^{\perp}, Z_{Y}\right\}$ are both orthonormal bases of $\mathfrak{g}$, so we may use either when calculating the wedge product.

Now

$$
\begin{aligned}
T \mathcal{V} \wedge \mathcal{F}= & \pm\left(-\lambda Z_{Y}, 0\right) \wedge\left(\frac{\tau x_{2}^{2}}{z_{0}^{2}} v_{1}^{\perp}, \tau_{2} x_{2}^{2} y \epsilon X\right) \wedge\left(-\tau v_{2}^{\perp}, \frac{\tau_{2} \lambda y x_{2} z_{0} \epsilon}{\tau} X\right) \\
& \wedge\left(\frac{-y \lambda \tau \epsilon}{\tau_{2}}\left(\frac{1+x_{2}^{2}}{z_{0}^{2}}\right) v_{1}^{\perp}-\frac{\tau^{2} y \epsilon x_{2}}{\tau_{2} z_{0}} v_{2}^{\perp}+\frac{x \lambda \tau \epsilon}{\tau_{2} z_{0}} U^{\perp}, 0\right) \\
& \wedge(0, Y) \wedge\left(0, Y^{\perp}\right) \wedge\left(0, Z_{Y}\right) \wedge\left(0, Z_{Y^{\perp}}\right) \\
& \wedge\left(-v, \frac{\tau_{2}^{2} x_{2} z_{0} y \epsilon}{\tau^{2}} X\right) \wedge\left(-U^{\perp}, \frac{\tau_{2} z_{0} x \epsilon}{\tau} X\right) .
\end{aligned}
$$

We use here the fact that since we wedge with $(0, Y) \wedge\left(0, Y^{\perp}\right) \wedge\left(0, Z_{Y}\right) \wedge$ $\left(0, Z_{Y^{\perp}}\right)$, only the coefficient of $X$ matters in the right-hand components.

Writing everything as a multiple of

$$
\begin{aligned}
\mathcal{V} \wedge \mathcal{E}= \pm(v, 0) & \wedge\left(v_{1}^{\perp}, 0\right) \wedge\left(v_{2}^{\perp}, 0\right) \wedge\left(U^{\perp}, 0\right) \wedge(0, X) \\
& \wedge\left(Z_{Y}, 0\right) \wedge(0, Y) \wedge\left(0, Y^{\perp}\right) \wedge\left(0, Z_{Y}\right) \wedge\left(0, Z_{Y^{\perp}}\right)
\end{aligned}
$$

we have a DG-Multiplier of

$$
\sqrt{\frac{1}{\lambda|\operatorname{det} A|}}=\frac{z_{0}}{\tau x_{2} \lambda}
$$


where

$$
A=\left(\begin{array}{ccccc}
0 & \frac{\tau x_{2}^{2}}{z_{0}^{2}} & 0 & 0 & \tau_{2} x_{2}^{2} \epsilon y \\
0 & 0 & -\tau & 0 & \frac{\tau_{2} \lambda y x_{2} z_{0} \epsilon}{\tau} \\
0 & \frac{-y \lambda \tau \epsilon\left(1+x_{2}^{2}\right)}{z_{0}^{2} \tau_{2}} & \frac{-\tau^{2} \epsilon x_{2} y}{z_{0} \tau_{2}} & \frac{x \lambda \tau \epsilon}{\tau_{2} z_{0}} & 0 \\
1 & 0 & 0 & 0 & \frac{-\tau_{2}^{2} x_{2} z_{0} y \epsilon}{\tau^{2}} \\
0 & 0 & 0 & 1 & \frac{-\tau_{2}^{2} z_{0} x \epsilon}{\tau}
\end{array}\right)
$$

Note that $0<x_{2}<1$ and $\lambda>0$.

\section{Conclusions}

The wave invariant calculations, particularly for the isospectral Heisenberg manifolds, show that for a given length $\tau$ in the length spectrum, both the number of free homotopy classes containing a closed geodesic of length $\tau$ and the volume of the initial velocities producing these closed geodesics are of paramount importance when computing the wave invariants. One might hope to adjust the definition of multiplicity to include the volumes of the $F(\tau, c)$ toward showing that all examples of isospectral (nil)manifolds must have the same length spectrum with this new multiplicity. However, by (2.17), the only multiplicity or weighting of each length that assures equality is precisely the weighting given by the wave trace.

\section{Acknowledgment}

The author wishes to thank Alejandro Uribe for inspiring conversations about and invaluable references for this paper.

\section{References}

[1] P. Anselone, Collectively compact operator approximation theory and applications to integral equations, Prentice Hall, Englewood Cliffs, NJ, 1971.

[2] M. Berger, P. Gauduchon and E. Mazet, Le spectre d'une variété Riemannienne, Lecture notes in mathematics, vol. 194, SpringerVerlag, Berlin and New York, 1971. 
[3] R. Brummelhuis, T. Paul, and A. Uribe, Spectral estimates around a critical level, Duke Math. J. 78 (1995), $477-530$.

[4] I. Chavel, Eigenvalues in Riemannian geometry, Including Chapter XI by Burton Randol. With an appendix by Jozef Dodziuk. Pure and applied mathematics, vol. 115, Academic Press, Orlando, FL, 1984 .

[5] Y. Colin de Verdière, Spectre du laplacien et longueurs des géodésiques périodiques, I-II, Compos. Math 27 (1973), 83-106, 159-184.

[6] J.H. Conway and J.P. Rossetti, Hearing the platycosms, Math. Res. Lett. 13 (no. 2-3) (2006), 475-494.

[7] J.H. Conway and J.P. Rossetti, Describing the platycosms (electronic preprint), arXiv:math/0311476v1 [math.DG].

[8] D. DeTurck and C.S. Gordon, Isospectral deformations I: Riemannian structures on two-step nilspaces, Comm. Pure Appl. Math. 40 (1987), $367-387$.

[9] D. DeTurck and C.S. Gordon, Isospectral deformations II: trace formulas, metrics, and potentials, Comm. Pure Appl. Math. 42 (1989), 1067-1095.

[10] J.J. Duistermaat, On the Morse index in variational calculus, Adv. Math. 21 (1976), 173-195.

[11] J.J. Duistermaat and V.W. Guillemin, The spectrum of positive elliptic operators and periodic bicharacteristics, Invent. Math. 29 (1975), 39-79.

[12] P. Eberlein, Geometry of two-step nilpotent groups with a left invariant metric, Ann. Sci. École Norm. Sup. (4)27, (1994), 611-660.

[13] P. Eberlein, Left invariant geometry of Lie groups, Cubo 6 (2004), 427-510.

[14] C.S. Gordon, The Laplace spectra versus the length spectra of Riemannian manifolds. Nonlinear problems in geometry (Mobile, Ala., 1985), 63-80, Contemp. Math. 51, Amer. Math. Soc., Providence, RI, 1986.

[15] C.S. Gordon, Riemannian manifolds isospectral on functions but not on 1-forms, J. Diff. Geom. 24 (1986), 79-96. 
[16] C.S. Gordon, Isospectral closed Riemannian manifolds which are not locally isometric, J. Differ. Geom. 37 (1993), 639-649.

[17] C.S. Gordon and E.N. Wilson, Isospectral deformations of compact solvmanifolds, J. Differ. Geom. 19 (1984), 241-256.

[18] C.S. Gordon and E.N. Wilson, The spectrum of the Laplacian on Riemannian Heisenberg manifolds, Michigan Math. J. 33 (1986), 253-271.

[19] C.S. Gordon and E.N. Wilson, Continuous families of isospectral Riemannian manifolds which are not locally isometric, J. Differ. Geom. 47 (1997), 504-529.

[20] R. Gornet, Equivalence of quasi-regular representations of two and three-step nilpotent Lie groups, J. Funct. Anal. 119 (1994), $121-137$.

[21] R. Gornet, The length spectrum and representation theory on twoand three-step nilpotent Lie groups, in Contemporary mathematics: geometry of the spectrum (Seattle, WA, 1993), eds. R. Brooks, C.S. Gordon, P. Perry, vol. 173, Amer. Math. Soc., 1994, Providence, RI, pp. $133-155$.

[22] R. Gornet, A new construction of isospectral Riemannian nilmanifolds with examples, Michigan Math. J. 43 (1996), 159-188.

[23] R. Gornet, The marked length spectrum vs. the Laplace spectrum on forms on Riemannian nilmanifolds, Comment. Math. Helv. 71 (1996), 297-329.

[24] R. Gornet, Continuous families of Riemannian manifolds, isospectral on functions but not on 1-forms, J. Geom. Anal. 10(no. 2) (2000), 281-298.

[25] R. Gornet, Riemannian nilmanifolds and the trace formula (electronic), Trans. Am. Math. Soc. 357 (2005), 4445-4479.

[26] R. Gornet and M. Mast, The length spectrum on two-step nilmanifolds, Ann. Sci. École Norm. Sup. (4) 33(no. 2) (2000), 181-209.

[27] R. Gornet and M. Mast, Length minimizing geodesics and the length spectrum of Riemannian two-step nilmanifolds, J. Geom. Anal. 13(no. 1) (2003), 107-143. 
[28] R. Gornet and M. Mast, The minimal marked length spectrum of Riemannian two-step nilmanifolds, Michigan Math. J. 52 (2004), 683-716.

[29] V. Guillemin, Wave-trace invariants, Duke Math. J. 83(no. 2) (1996), 287-352.

[30] V. Guillemin and A. Uribe, On the de Haas-van Alphen effect, Asymptot. Anal. 6 (1993), 205-217.

[31] A. Kaplan, Riemannian nilmanifolds attached to Clifford modules, Geom. Dedicata 11 (1981), 127-136.

[32] T. Kato, A short introduction to perturbation theory for linear operators, Springer-Verlag, New York and Berlin, 1982.

[33] J. Miatello and J.P. Rossetti, Length spectra and p-spectra of compact flat manifolds, J. Geom. Anal. 13 (2003), 631-657.

[34] He Ouyang, On isospectral rigidity of deformations on two-step nilmanifolds, PhD thesis, Washington University in St. Louis, 1991.

[35] H. Pesce, Déformations isospectrales de certaines nilvariétés et finitude spectrale des variétés de Heisenberg, Ann. Sci. École Norm. Sup. (4) 25 (1992), 515-538.

[36] H. Pesce, Déformations L-isospectrales sur les nilvariétés de rang deux, C. R. Acad. Sci. Paris Sér. I Math. 315 (1992), 821-823.

[37] H. Pesce, Calcul du spectre d'une nilvariété de rang deux et applications, Trans. Am. Math. Soc. 339 (1993) 433-461.

[38] H. Pesce, Une formule de Poisson pour les variétes de Heisenberg, Duke Math. J. 73(no. 1) (1994), 79-95.

[39] M.S. Raghunathan, Discrete subgroups of Lie groups, Ergebnisse der Mathematik und ihrer Grenzgebeite. vol. 68, Springer-Verlag, New York and Heidelberg, 1972.

[40] A. Selberg, Harmonic analysis and discontinuous groups in weakly symmetric Riemannian spaces with applications to Dirichlet series, J. Indian Math. Soc. (N.S.) 20 (1956) 47-87.

[41] S.R. Simanca, Pseudo-differential operators, Pitman research notes in mathematics series., vol. 236, Longman Scientific \& Technical, Harlow; copublished in United States with John Wiley \& Sons, New York, 1990. 
[42] R. Strichartz, A guide to distribution theory and Fourier transforms, Studies in Advanced Mathematics, CRC Press, Boca Raton, FL, 1994.

[43] A. Uribe, Trace formulae, Contemporary mathematics, first summer school in analysis and mathematical physics (Cuernavaca Morelos, 1998), vol. 260, American Mathematical Society, Providence, RI, 2000, pp. $61-90$.

[44] V.S. Varadarajan, Lie groups, Lie algebras, and their representations, Graduate texts in mathematics, vol. 102, Springer-Verlag, New York, 1984.

[45] S. Zelditch, Lectures on wave invariants, Spectral theory and geometry (Edinburgh, 1998), London. Math. Soc. Lecture Note Ser., vol. 273, Cambridge University Press, Cambridge, 1999, pp. 284-328.

University of Texas at Arlington

Department of Mathematics

Arlington, Texas 76019-0408

USA

E-mail address: rgornet@uta.edu

Received December 29, 2005 